\title{
INVESTIGATIONS ON mRNA MATURATION DISTURBANCIES IN THE PATHOGENESIS OF PSORIASIS
}

\author{
Ph.D. Thesis
}

Eszter Szlávicz MD

Department of Dermatology and Allergology

Doctoral School of Clinical Medicine Faculty of Medicine University of Szeged

Supervisor: Prof. Dr. Márta Széll

Szeged

2017 


\section{LIST OF PUBLICATIONS}

\section{Publications directly related to the subject of the thesis:}

I. Szlavicz E, Szabo K, Groma G, Bata-Csorgo Z, Pagani F, Kemeny L, Szell M (2017) Analysis of psoriasis-relevant gene expression and exon usage alterations after silencing of SR-rich splicing regulators. Article submitted to Experimental Dermatology, under review. IF: $\mathbf{2 , 6 8}$

II. Szlavicz E, Szabo K, Groma G, Bata-Csorgo Z, Pagani F, Kemeny L, Szell M (2017) Splicing factors differentially expressed in psoriasis alter mRNA maturation of disease-associated EDA+ fibronectin Molecular and Cellular Biochemistry, doi: 10.1007/s11010-017-3090-1 IF: 2,67

III. Szlavicz E, Szabo K, Bata-Csorgo Z, Kemeny L, Szell M (2014) What have we learned about non-involved psoriatic skin from large-scale expression studies? World Journal of Dermatology, 3(3):50-57. doi: 10.5314/wjd.v3.i3.50

\section{Publications not related to the subject of the thesis:}

I. Szlavicz E, Perera PS, Tomboly C, Helyes Z, Zador F, Benyhe S, Borsodi A, Bojnik E (2015) Further Characterization of Hemopressin Peptide Fragments in the Opioid and Cannabinoid Systems. Anesthesia and Analgesia, 121(6):1488-94. doi:

10.1213/ANE.0000000000000964. IF: 3,83

II. Zador F, Samavati R, Szlavicz E, Tuka B, Bojnik E, Fulop F, Toldi J, Vecsei L, Borsodi A (2014) Inhibition of opioid receptor mediated G-protein activity after chronic administration of kynurenic acid and its derivative without direct binding to opioid receptors. CNS Neurol Disord Drug Targets. 13(9):1520-9. doi:

10.2174/1871527314666141205164114 IF: 2,63 


\section{TABLE OF CONTENTS}

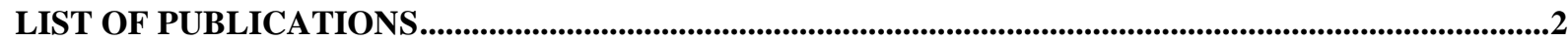

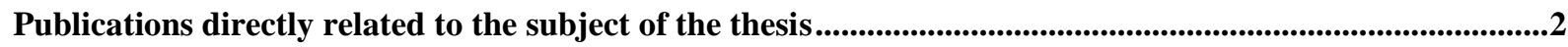

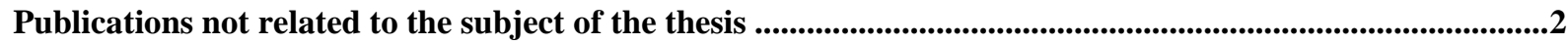

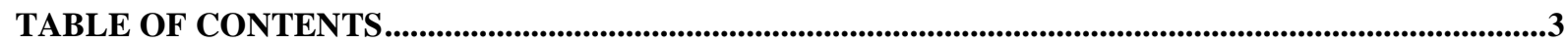

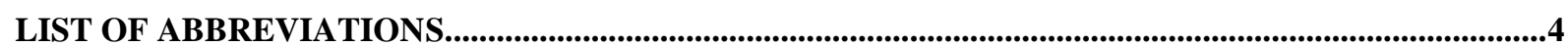

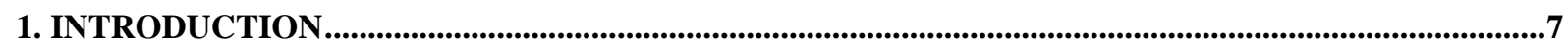

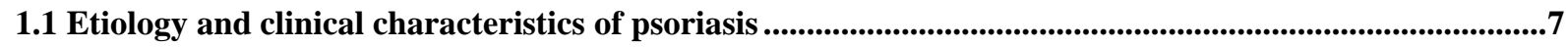

1.2 Molecular background of psoriasis pathogenesis.............................................................................................9

1.3 EDA+fibronectin and its potential impact in psoriasis pathogenesis .......................................................11

1.4 High-througput methods in the identification of psoriasis-related signalling mechanisms .....................13

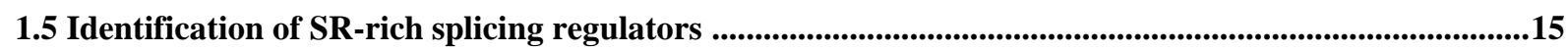

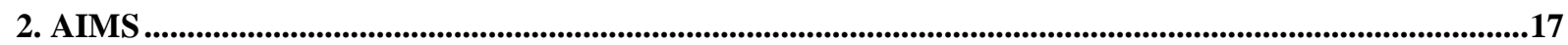

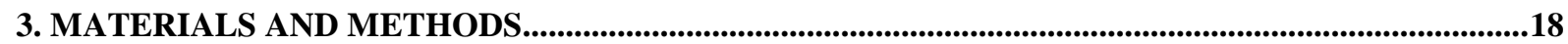

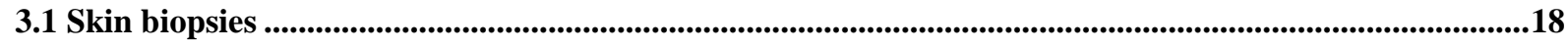

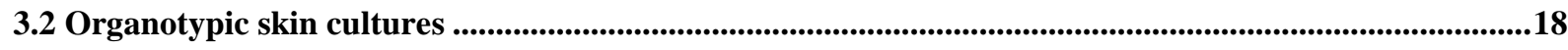

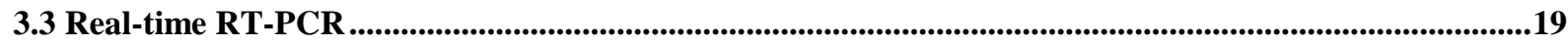

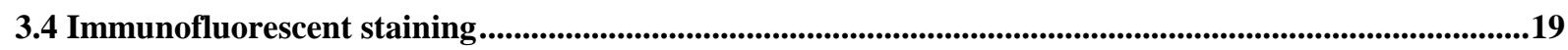

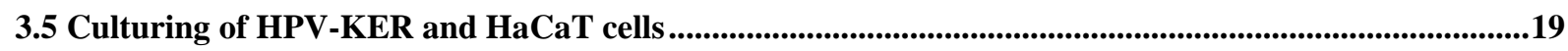

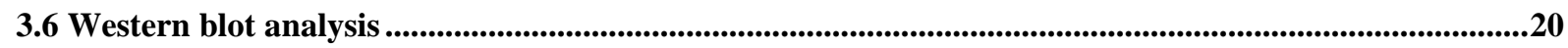

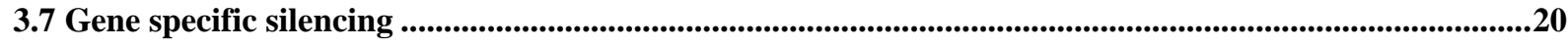

3.8 Polymerase chain reaction .......................................................................................................................................21

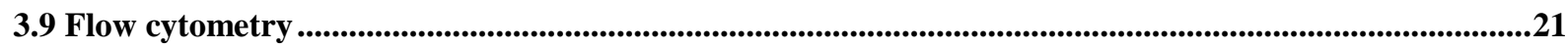

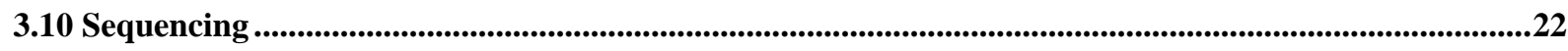

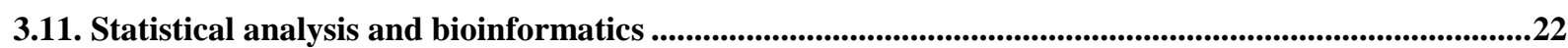


4.1 SR-rich splicing factor genes exert decreased response to T-lymphokine stimuli in psoriatic non-involved epidermis as compared to healthy samples.

4.2 LUC7L3, PPIG and SFRS18 show altered expression in psoriasis

4.3 LUC7L3, PPIG and SFRS18 exhibit similar pattern of expression in synchronized, immortalized keratinocytes. .26

4.4 siRNA silencing of PPIG, SFRS18 and LUC7L3 gene expression alter the ratio of EDA+/total fibronectin .28

4.5. Global transcriptome analysis of immortalized keratinocytes 31

4.5.1 Determination of silencing efficacies and quality control 31

4.5.2 Paired-end RNA-Sequencing reveals differential expression and exon usage patterns upon the silencing of the LUC7L3 and SFRS18 splicing factors 


\section{LIST OF ABBREVIATIONS}

IIICS: type III connecting segment

CCL: chemokine ligand

CI: confidence interval

CRE: cAMP responsive element

CUL1: cullin-1

DC: dendritic cell

DEG: differentially expressed genes

DMEM: Dulbecco's modified Eagle's medium

EDA: extra domain A

EDB: extra domain B

FACS: fluorescence-activated cell sorting

FC: fold-change

FN1: fibronectin

GO: gene ontology

GM-CSF: granulocyte-macrophage colony-stimulating factor

GWAS: genome wide association studies

HERC: HECT and RLD domain containing

HLA: human leukocyte antigene

HPV: human papilloma virus

IFI: interferon-induced

IFN: interferon

IL: interleukin

ISGs: interferon-stimulated genes

KER: keratinocyte

KGF: keratinocyte growth factor

IncRNAs: long non-coding RNAs

LUC7L3: luc-7 like protein 3 
MHC: major histocompability complex

NBEAL1: neurobeachin like 1

NEAT1: nuclear paraspeckle assembly transcript 1

OAS2 2'-5'-oligoadenylate synthetase 2

PBS: phosphate-buffered saline

PPIG: peptidyl-prolyl cis-trans isomerase $\mathrm{G}$

PASI: psoriasis area and severity index

PNISR: PNN interacting serine and arginine rich protein

PNN: pinin

RIN: RNA integrity number

RNA-Seq: RNA-Sequencing

RT-PCR: reverse transcription polymerase chain reaction

SDC4: syndecan-4

SDS-PAGE: sodium dodecyl sulfate polyacrylamide gel electrophoresis

SE: standard error

SFM: serum-free medium

SFRS18: splicing factor, arginine/serine-rich 18

SiRNA: small interfering RNA

SRPK: serine/arginine-rich splicing factor kinase

STAT1: signal transducer and activator of transcription 1

TGF- $\beta$ : transforming growth factor beta

Th: T-helper

TINCR: tissue differentiation-inducing non-protein coding RNA

TLR: toll-like receptor

TNC: tenascin-c

TNF: tumor-necrosis factor

WGCNA: weighted correlation network analysis 


\section{INTRODUCTION}

\subsection{Etiology and clinical characteristics of psoriasis}

Psoriasis is one of the most common chronic inflammatory skin disorders, affecting appr. 2\% of the population. In Europe, its prevalence is between 1,6\% and 4,8\% [1, 2]. Considering disorder onset, a dual peak can be observed: approximetely $75 \%$ of patients are under the age of 40 years (type I), and usually exhibit a more serious prognosis in contrast to late onset cases (type II, between ages 55-60) [3]. Psoriasis vulgaris is the most frequent manifestation, which accounts for appr. 80-95\% of all cases; the characteristic lesions of the disorder are the erythematosus, scaly, demarcated and squamousus plaques, which could cover predominantly the skin of knees, elbows and the scalp (Fig. 1., a-c). However, multiple clinical forms exist besides the well-known plaque-type disorder, with various morphology and localization of the symptoms: the different phenotypes consist of guttate, pustular (acrodermatitis continua of Hallopeau, palmoplantar and general pustulosis), inverse and erythrodermic psoriasis $[1,4,5]$. Cutaneous histopathological features of the symptoms are also typical (Fig. 1. d-e), containing hyper- and parakeratosis, acanthosis, dilatation of blood vessels and collection of neutrophil granulocytes (Munro's microabscesses) [5, 6].

The negative impact of psoriasis on life quality is self-explanatory, and there is still a discussion whether it should be considered a skin or a systematic disorder. Joint involvement is present in a significant number of cases (approximatly one fifth of patients, between 10-34\%), moreover, cardiovascular comorbidities are also frequent in psoriasis, and several psoriatic patients suffer from the consequences of metabolic syndrome [2, 4, 7]. Assocation with immune-mediated, autoimmune disorders, including inflammatory bowel disease, Hashimoto's thyreoditis and multiple sclerosis has been also reported [7]. In addition, psoriasis is associated with serious psychosomatic stress, and concomitant psychiatric diseases, such as anxiety and depression, the latter one affecting appr. $60 \%$ psoriatic cases $[4,8,9]$. 

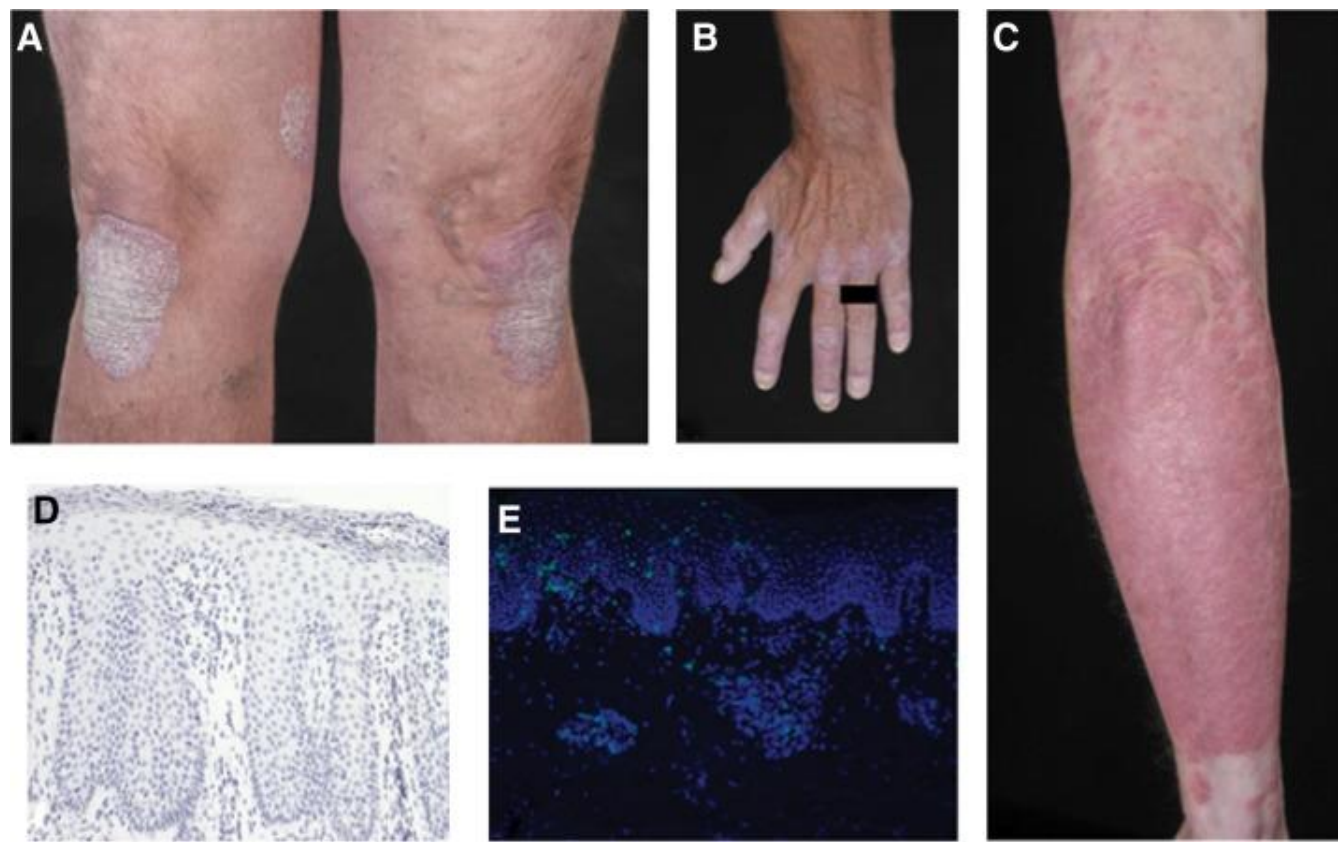

Fig. 1. Characteristic clinical presentations of psoriasis, including psoriatic plaques and nail involvement (a-c). Histopathologic features include acanthosis, papillomatosis and Munro's microabscesses (d) and the infiltrates of CD3+ T-lymphocytes (e) (Di Meglio et al., 2013)

Regarding disorder development, we should emphasize a multifactorial origin, in which genetic and environmental factors are also determinant. Twin studies shed light on the importance of genetic susceptibility of psoriasis and reported 2-3,5 fold higher risk for monozygotic twins as compared to dizygotic ones $[1,5]$. Classic genome-wide linkage analysis and Genome Wide Association Studies (GWAS) provide valuable data about the genetic loci predisposing to psoriasis development. According to our current understanding, a major portion (approximately $35-50 \%$ ) of heritability is related to the PSORS1 loci, which includes genes of the major histocompability complex (MHC), encoding the human leukocyte antigens (HLAs) [1]. It is well documented, that within the MHC, the HLA-C*06:02 allele presents the greatest risk for psoriasis, accompanied by more serious manifestation and early disease onset $[1,4,10$, 11]. Besides PSORS1, numerous other genetic susceptibility factors have been described, also containing keratinocyte-derived and both adaptive and innate immunity-related components, however the majority of publications suggest that the contribution of these genomic regions to psoriasis formation are relatively modest $[4,5,11]$. On the other hand, rare mutations of the 
IL36RN and CARD14 genes could result in considerable effects [6]. Among environmental triggers, the involvement of mechanical trauma, infections, certain medications, UV-exposure, smoking, stress and some alimentary factors are the most notable ones. The Köbner phenomenon demontrates the importance of mechanical distress as a promoter of skin symptoms $[1,4,12,13,14,15]$.

\subsection{Molecular background of psoriasis pathogenesis}

To date, conceptions of psoriasis underwent serious changes: the current reports emphasize the critical role of the IL23/IL17/IL22 axis and TNF- $\alpha$, as opposed to the previous idea that psoriasis is a Th1 and IFN- $\gamma$ dominated disorder $[4,16,17,18,19]$. Their importance was also supported by studies specifically targeting these cytokines [6, 19, 20, 21, 22, 23]. However, the molecular background of psoriasis is quite complex, positive and negative feedback mechanisms and amplification signals are substantial in the disorder pathogenesis. Thus, psoriasis cannot be characterized by a linear pathway, as interactions of cytokines constitute a rather extensive, self-sustaining network, where either innate or adaptive immune system is involved, and the reciprocal actions of keratinocytes, different types of dendritic cells and Tlymphocytes, as well as other inflammatory cellular elements account for layers of molecular complexity [5, 6]. Moreover, cytokine products showing a characteristic distribution during the disease course, while the disorder initiation is dominated by a Th1 profile, in the maintained chronic inflammatory phase, Th17 cytokines gain an emerging importance $[4,5]$.

Despite the advances in the field of psoriasis research, molecular triggers and the mechanism of disorder initiation are still only partially understood, and many questions remain that need to be answered [6]. Literature data support that the activation of a circulatory dendritic cell (DC) subset, the plasmacytoid DCs could be a determinant event in the early pathogenic steps. In healthy skin, pDCs are almost absent, and they are stimulated via their TLR7/9 receptors, which interact with the complexes of the self DNA/RNA derived from stressed keratinocytes and cathelicidin (LL37), one of the so-called antimicrobial peptides (AMPs) [4, 24, 25, 26]. AMPs are cationic antimicrobial peptides, their release from keratinocytes are critical components of 
the innate immune response in psoriasis. Besides LL37, S100 proteins are also important in psoriasis pathogenesis, since they amplify inflammatory processes due to activation of TLR receptors and the chemotactic feature $[6,27,28]$.

In the further pathogenic steps, the stimulated pDCs release type I interferons $[4,26,29]$. Type I interferons along with the keratinocyte-derived mediators such as IL-1 $\beta$, IL-6 and TNF $\alpha$ facilitate maturation and activation of the myeloid dermal dendritic cells (DDCs) [4, 19, 20, 26]. Furthermore, this step provides the connection of innate and adaptive immune processes, since DDCs are the producers of IL12, which stimulates the Th1 subset of keratinocytes and leads to the release of IFN- $\gamma$. On the other hand, another important DDC and macrophagederived cytokine, IL23 triggers the Th17 and Th22 T-cell lineages [1, 4, 19]. It is welldocumented, that in the interaction of DDCs and T-cells, presence of TNF- $\alpha$ is essential $[6,20]$. The Th17 products promote T-cell (CCL20) and neutrophil (CXCL1, CXCL2, CXCL5, CXCL8) recruiting factors; LL37 and S100 protein production is also facilitated, perpetuating the inflammatory circuit $[4,30]$. In addition, IL22 induces the psoriasis-specific accumulation of the innate keratinocytes $[6,31]$.

A schematic of the pathogenic events is demonstrated in Fig. 2. Besides the listed pathogenic events, autocrin loops, recruitement of tissue-resident memory $\mathrm{T}$ cells and release of angiogenic/vasoactive mediators (leading to punctual bleedings after removement of scales, called the Auspitz-sign) are also important components of the disorder development [4, 5, 19, $32,33,34]$. In summary, as a consequence of the above detailed molecular processes, the turnover time of keratinocytes shows considerable accelaration compared the normal approximately 40 days, and the immature psoriatic keratinocytes reach the skin surface altogether in 6-8 days $[10,35]$. The altered response of keratinocytes to the T-cell signals is presumably an essential factor of psoriasis pathogenesis, however, the exact mechanism of disorder initiation is still unclear. 


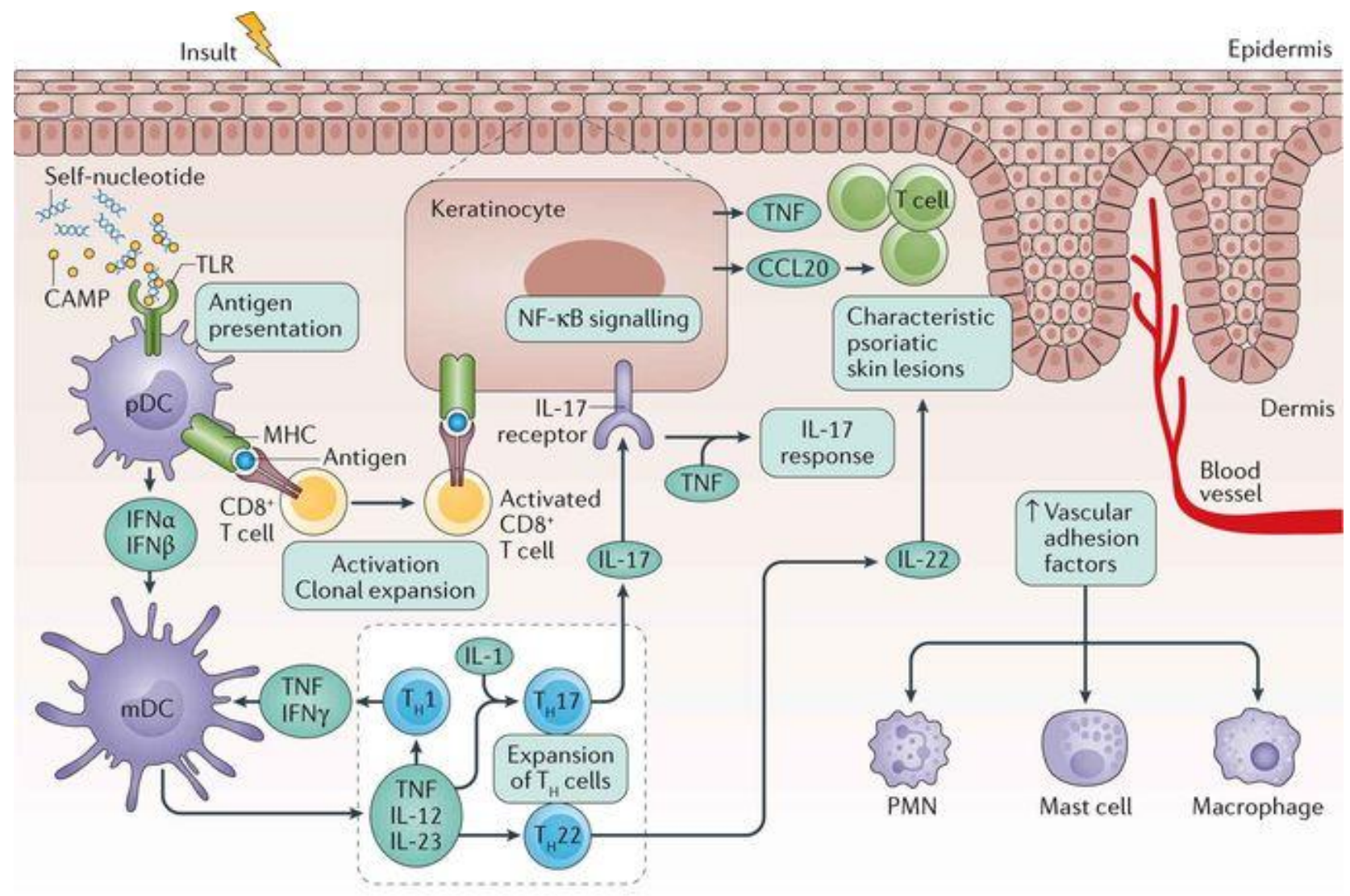

Fig. 2. Summary of initiatory pathogenic steps and the molecular background of the established psoriasis disorder (Greb et al., 2017)

\subsection{EDA+ fibronectin and its potential impact in psoriasis pathogenesis}

To date, relatively few studies have been conducted on mRNA maturation disturbances in psoriasis. One of the first reports established that the extracellular matrix protein tenascin-C showed differences in the inclusion of fibronectin type III fibronectin modules. The study found that the larger tenascin- $\mathrm{C}$ variant is more abundant in both epidermal keratinocytes and fibroblasts, altough the relative amount of the smaller transcript is higher in epidermal keratinocytes as compared to the fibroblasts. IFN- $\gamma$, TNF- $\alpha$ and IL4 have been described as regulators of the splicing process, and protein-level distribution of tenascin-C splice variants was also examined in healthy skin, wounds, psoriatic lesions and epidermal tumours [36]. Moreover, in the study of Ting et al. altered splicing patterns for another type III fibronectin 
module were demonstrated: it was shown that EDA+ domain of the extracellular matrix protein fibronectin is overexpressed in psoriatic non-involved epidermis [37]. The study suggested that $\mathrm{EDA}+$ fibronectin might be a putative factor which makes epidermal keratinocytes prone to the proliferative stimuli of the professional immune cells.

Although fibronectin is a suitable model for analyzing mRNA maturation processes, the complete mechanism of splicing regulation as well as the EDA+ domain inclusion are not fully revealed. The production of splice variants has been shown to be an important point of regulation for several genes, including fibronectin, which produces at least $20 \mathrm{mRNA}$ variants as a result of alternative splicing $[38,39,40]$. As we mentioned previously, the EDA domain belongs to the Type III repetitive modules (Fig. 3.) of fibronectin, together with EDB and Type III connecting segment (IIICS) [38, 39, 41]. The EDA module is excluded from plasma fibronectin, whereas cellular fibronectin could include variants with the EDA domain [41, 42]. $\alpha 5 \beta 1$ integrin is the classic receptor for fibronectin, but splicing events could impact the fibronectin related signalling: in case of the EDA+ variant, TLR4 binding is well-established $[41,42,43,44,45,46]$.

$\mathrm{EDA}+$ fibronectin is typically present during embryonic development, nevertheless, there is a body of evidence indicating that this splice variant plays an important role in wound healing, inflammatory, angiogenic and malignant processes in adults [38, 47, 48, 49]. Besides the results of Ting et al., our research group has also demonstrated that EDA+ fibronectin could play a crucial role in psoriasis pathogenesis: we found that proliferating keratinocytes (both normal cultured keratinocytes and $\mathrm{HaCaT}$ cells) are able to produce this isoform [37, 50]. In addition, it has also been established that EDA+ fibronectin expression was modest in healthy keratinocytes whereas keratinocytes from psoriatic non-involved epidermis proved to be effective producers of the splice variant. The ratio of the normal/oncofetal fibronectin isoforms depends on the proliferation and differentiation states, and a peak of total and EDA+ fibronectin expression was observable in highly proliferating cells. These data supported the theory that EDA+ fibronectin promotes the sensitization of keratinocytes to the mitogenic stimuli of professional immune cells [50]. 


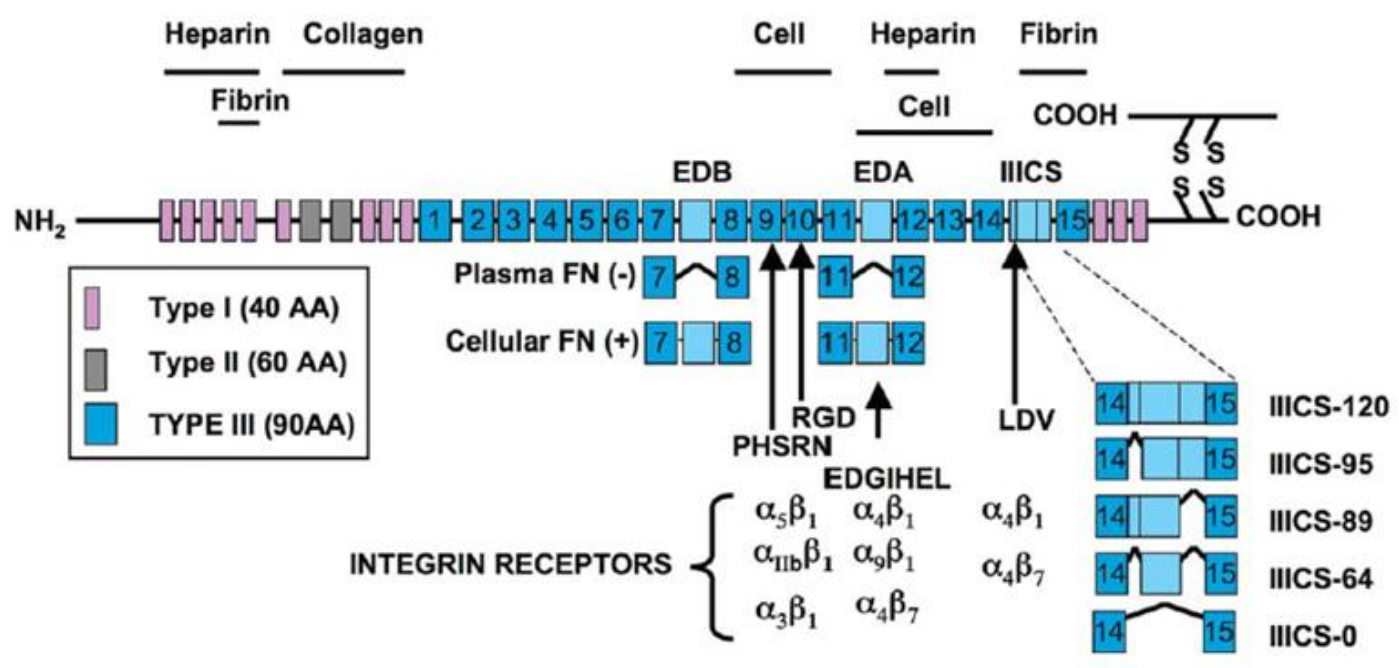

Fig. 3. Structure of fibronectin gene. The EDA+ domain belongs to the type III repetative moduls, such as EDB and Type III connecting segment (IIICS) (White et al., 2008).

\subsection{High-througput methods in the identification of psoriasis-related signalling mechanisms}

In recent years, several powerful methods have been developed for large-scale gene expression profiling. Among these tools, the most widespread were the cDNA microarrays and via the evolution of next-generation sequencing, pair-end RNA-Sequencing gained an emerging importance in experimental dermatology as well [51, 52, 53, 54].

The recent cDNA microarrays provided remarkably valuable information about the gene expression profiles of healthy, psoriatic non-involved and involved epidermis and they were also able to verify discrepancies related to the innate and adaptive immune responses. Besides altered immune functions, these studies emphasized the pathogenic role of epidermal keratinocyte proliferation, apoptosis and lipid metabolism related genes. Considering immunological processes, the importance of the proinflammatory T-cell lymphokine products such as INF- $\gamma$ and IL17, or genes encoding chemokines and their receptors was also demonstrated in psoriasis development by the recent cDNA microarray experiments $[55,56$, 
$57,58,59,60]$. Wnt pathway was found to be significantly elevated in the involved epidermis compared to non-involved samples, verifying the effects on cellular proliferation and differentiation [61]. In addition, other data supported the involvement of mTOR, NFאB, BCL2 and $\mathrm{BAX}[62,63]$. PPAR $\delta$, the inflammatory and metabolic process-related transcription factor, is also implicated in the induction of keratinocyte proliferation, and exhibits proangiogenic effects as well [64]. Other research groups have found down-regulation of lipid metabolism-related pathways in non-involved samples and further downregulation was observed in the involved skin [58]. Considering that the results of former cDNA microarrays show distinct variance across the experiments, meta-analyses of the available datasets were also carried out. These studies identified among the most significant molecular pathways cellular proliferation/differentiation and immunomodulation, respectively. Moreover, meta-analyses confirmed the importance of metabolic disturbances, including atherosclerosis-related molecular abnormalities and transcripts associated with impaired glucose tolerance $[65,66]$.

Deep RNA-Sequencing harbors numerous advances as compared to microarrays, and eventually were put to general use in psoriasis research as well. In contrast to limitations of microarrays, RNA-Seq generates relatively low background noise and has a wide dynamic range, in addition, it does not require known template sequences to transcript abundance identification [54, 67]. One of the first reports was published by Jabbari and co-workers, where three pairs of psoriatic non-involved and involved biopsies were compared, and at the same time, the samples underwent microarray hybridization, as well. Their results indicated that RNA-Seq is capable of identifying low abundance transcripts, thus, a larger set of differentially expressed transcripts (DEGs) could be detected as compared to microarrays [67]. Li et al. worked with a larger sample size as they sequenced polyadenylated RNA of 92 psoriatic patients and 82 healthy volunteers. Consistent with previous microarray data, they could display up-regulation of inflammatory response-related genes, including Th17 associated genes and IFN- $\gamma$; and the upregulation of cell proliferation, and keratinization was also supported by this study [68].

Due to advances of RNA-Seq technology, splicing pattern detection and measurement of noncoding transcript abundance are also available. Li et al. has already reported differential usage of 343 exons among healthy and psoriatic non-involved samples [68]. In a recent report, a different approach was used and compared the abundance of distinct RNA isoforms, identifying 
more than 9000 differential RNA isoforms, which are present in different amount between healthy, psoriatic non-involved and involved epidermis [69]. Considering non-coding transcripts, Antonini et al. introduced the most important long non-coding RNAs (lncRNAs) identified in the course of dermatological research using high-throughput RNA-Sequencing and they also discussed the advantages and limitations of the examinations of lncRNAs [70].

\subsection{Identification of SR-rich splicing regulators}

The vast majority of former cDNA microarray experiments analyzed the steady-state gene expression pattern of two or three disctinct states, which includes healthy, psoriatic noninvolved and involved epidermis samples. Paralelled with other works published in the same field, the approach of our research group was quite different. The aim of our cDNA microarray study was the examination of the T-lymphokine induced gene expression changes, considering that altered keratinocyte responsiveness of psoriatic non-involed epidermis is essential in the early pathogenesis of psoriasis [71].

In the applied experimental setup, organotypic cultures were generated from both healthy and psoriatic epidermis samples, and half of them were treated with a lymphokine mixture containing GM-CSF, IFN- $\gamma$ and IL-3. It was previously proven that IFN- $\gamma$ in the presence of GM-CSF and IL-3 is able to promote proliferation of the keratinocyte precursors originating from the non-involved epidermis, therefore, these mediators could play a crucial role in the early steps of disorder development [72]. In the following analysis, a comparison has been made between the autologuous pairs of untreated and treated samples. After that, differentially expressed transcripts which showed a fold change $>2$ have been selected for further characterization and a comparisons have been made to identify differentially regulated genes among healthy and psoriatic non-involved epidermis samples [71]. To facilitate better understanding, the experimental design and comparisons applied during evaluation of the cDNA microarray are illustrated in a flow-chart (Fig. 4.). 


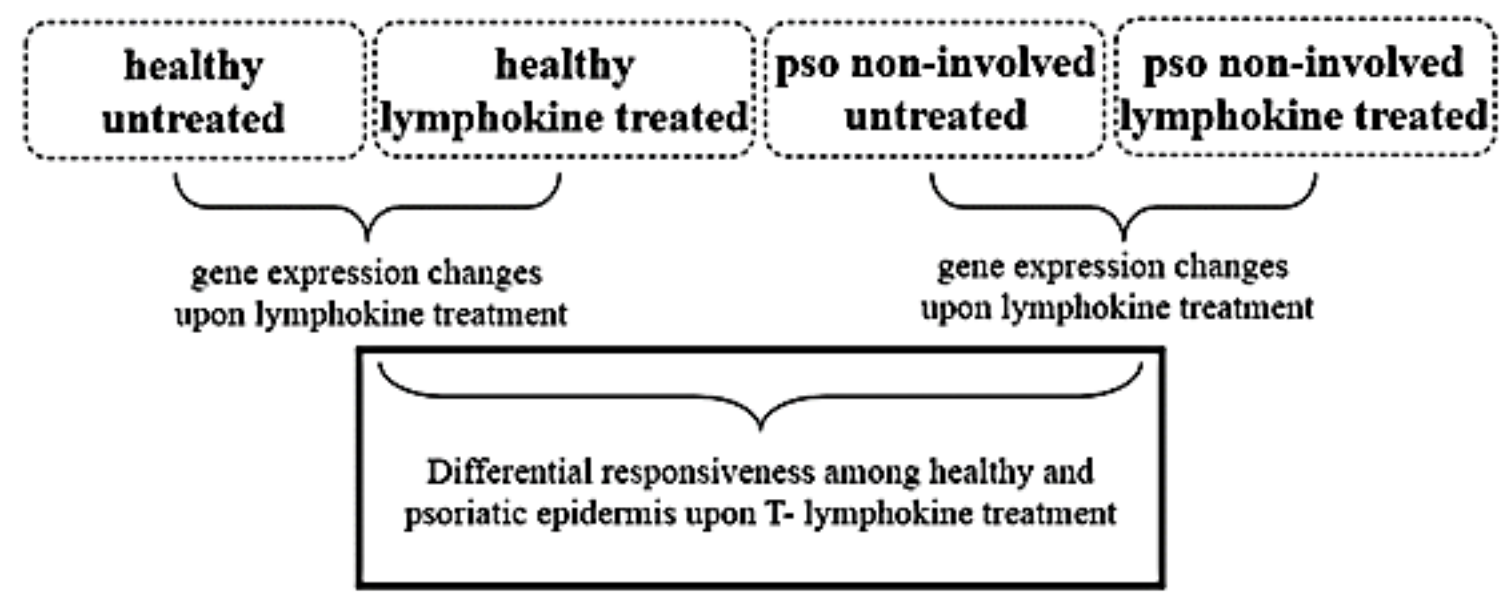

Fold-change $>2, p<0.05,95 \% \mathrm{CI}$

Fig. 4. Experimental set-up and the evaluation process of cDNA microarray results

An important outcome of the study was that due to T-lymphokine treatment, several identified genes showed upregulation in healthy epidermis, while in the non-involved epidermis, downregulation or unchanged gene expression was experienced. In addition, we could demonstrate by in silico analysis using publicly available databases, that the most influenced cellular processes are apoptosis and the small-lipid metabolism [71]. Moreover, we have also proved that certain SR-rich splicing regulators showed altered responsiveness to T-lymphokine stimuli. In this experiment, we identified splicing factor, luc-7 like protein 3 (LUC7L3), peptidyl-prolyl cis-trans isomerase G (PPIG) and arginine/serine-rich 18 (SFRS18), differentially regulated among healthy and psoriatic non-involved epidermis, therefore they could contribute to the responsiveness changes of keratinocytes. Since mRNA maturation disturbances of psoriasis were a limitedly studied topic so far, and our previuos results regarding the EDA+ fibronectin indicated that certain splicing abnormalities might contribute to psoriasis pathogenesis, we decided to perform further experiments related to the identified splicing factors. 


\section{AIMS}

1. Characterization of luc-7 like protein 3 (LUC7L3), peptidyl-prolyl cis-trans isomerase $\mathrm{G}$ (PPIG) and arginine/serine-rich 18 (SFRS18) splicing factor gene expression differences in psoriasis and during the proliferation /differentiation states of keratinocytes.

2. Examination of $L U C 7 L 3, P P I G$ and SFSR 18 influence on the mRNA maturation process of the extracellular matrix protein fibronectin.

3. To study additional gene expression and mRNA maturation alterations upon the modified expression of splicing factors in human keratinocytes 


\section{MATERIALS AND METHODS}

\section{$\underline{\text { 3.1 Skin biopsies }}$}

Previous cDNA microarray experiments were performed on organotypic skin cultures, which were established from tissue samples of 4 healthy volunteers and 4 psoriasis patients (age 1860 years). Patients enrolled in the study suffered from moderate-to-severe chronic plaque-type psoriasis, in each case Psoriasis Area and Severity Index (PASI) score was calculated by dermatologists. Another criterion for enrollement was a medication-free period $(\geq 2$ weeks without local therapy and/or $\geq 4$ weeks without systemic therapy). Tissue biopsies were obtained after the informed consent of participants. Tissue collection was carried out according to the guidelines of the Declaration of Helsinki and was approved by the Regional and Institutional Research Ethics Committee (2799, 3517).

\subsection{Organotypic skin cultures}

Organotypic skin cultures were generated from half cuts from shave biopsies [71]. First, epidermis samples were placed on $2.2 \mu \mathrm{m}$ porosity cellulose acetate/cellulose nitrate filters (Millipore) and after that, they were transferred to a stainless steel grid platform, in a 6-well plate. Dulbecco's modified Eagle's medium (DMEM) was used as culture medium, which was supplemented with $12 \mathrm{mM}$ glutamine and antibiotics (100 units $/ \mathrm{ml}$ penicillin and $100 \mu \mathrm{g} / \mathrm{ml}$ streptomycin), all of them were obtained from Life Technologies (Carlsbad, CA). In case of all donor skin specimens, a lymphokine mixture was added to one half of the organotypic cultures containing of $1 \mathrm{ng} / \mathrm{ml} \mathrm{IFN} \gamma, 1 \mathrm{ng} / \mathrm{ml} \mathrm{GM-CSF}$ and $0.3 \mathrm{ng} / \mathrm{ml} \mathrm{IL-3}$. The other half of the samples remained untreated. In order to the maintain organotypic skin cultures, standard culturing conditions were applied (at $37^{\circ} \mathrm{C}$ in a $5 \% \mathrm{CO}_{2}$ atmosphere), at the air/liquid interface for $72 \mathrm{~h}$ $[71,72]$. Following these steps, separation of the epidermis from the dermis was executed by overnight incubation in Dispase solution (grade II, Roche Applied Science) at $4^{\circ} \mathrm{C}$, finally, the samples were placed in TRIreagent (Molecular Research Center Inc., Cincinnati, OH). 


\subsection{Real-time RT-PCR}

Total RNA was purified from the organotypic skin cultures, and immortalized cell lines, such as HPV-immortalized keratinocytes (HPV-KER), HaCaT cells, respectively. One microgram of total RNA was reverse transcribed by the iScript TM cDNA Synthesis kit (\#1708891, BioRad, Hercules, CA), based on the protocol of manufacturers. To determine the transcript abundance, Real-Time RT-PCR was performed using custom primer sets, Universal Probe Library (Roche, Basel, Switzerland) and iQ Supermix (\#1708862, Bio-Rad, Hercules, CA). Relative gene expression was calculated using the $\Delta \Delta \mathrm{Ct}$ method, normalization process was done with the expression data of the $18 \mathrm{~S}$ ribosomal RNA.

\section{$\underline{\text { 3.4 Immunofluorescent staining }}$}

Biopsies from healthy, psoriatic non-involved and involved skin were frozen, embedded, and stored at $-80{ }^{\circ} \mathrm{C}$, before cutting into $6-\mu \mathrm{m}$ sections. Primary antibodies used during immunfluorescent stainings were as follows: anti-LUC7L3 (1:300, Abcam, Cambridge, UK), anti-PPIG (1:300; Abcam) and anti-SFRS18 (1:250, Novus Biologicals, Littleton, USA). In addition, we used Anti-mouse IgG-Alexa Fluor 647 and anti-rabbit Alexa Fluor 546 at 1:500 dilution as secondary antibodies (Life Technologies, Carlsbad, CA). For negative staining controls, sections were incubated with normal rabbit IgG (Santa Cruz Biotechnology, Dallas, TX, USA) or without the primary antibody (in case of PPIG). Nuclear staining was carried out with 2-(4-amidinophenyl)-1H-indole-6-carboxamidine (DAPI; Sigma-Aldrich, St Louis, MO, USA). Fluorescent intensity quantification of microscopic images was performed using ImageJ software: during evaluation, corrected-total-cell-fluorescence values were calculated based on integrated density of stained cell areas.

\subsection{Culturing of HPV-KER and HaCaT cells}

The HPV-KER cell line immortalization was achieved by the HPV E6 oncogene, description was provided by Polyanka et al., 2011 [73]. HPV-KER cells were cultured in $75 \mathrm{~cm}^{2}$ flasks (culturing conditions: $37^{\circ} \mathrm{C}$; humidified atmosphere containing $5 \% \mathrm{CO} 2$ ) and maintained in 
$1 \%$ antibiotic/antimycotic solution (PAA, Pasching, Austria) and 1\% L-glutamine (PAA) supplemented keratinocyte serum-free medium $\left(\right.$ Gibco $^{\circledR}$ Keratinocyte SFM Kit; Life Technologies, Copenhagen, Denmark). The medium of cells was changed every two days. The synchronization process was as follows: after confluence was obtained, the HPV-KER cells were maintained for one week in glutamine- and growth factor-free keratinocyte SFM. Cells were harvested with brief trypsinization ( $0.25 \%$ trypsin solution Sigma Aldrich, St. Louis, MO, USA). HaCaT cells were kindly provided by Dr. Fusenig, N.E. (Heidelberg, Germany). Cells were cultured and synchronized as described previously [74].

\section{$\underline{\text { 3.6 Western blot analysis }}$}

Equal amounts of proteins were separated on a 10\% SDS-PAGE gel and were transferred to Pure Nitrocellulose Membrane (Bio-Rad Laboratories) in the course of western blot analysis. Primary antibodies used in our experiments and their dilutions: anti-LUC7L3 (1:300, Abcam, Cambridge, UK), anti-PPIG (1:300, Abcam), anti-SFRS18 (1:300, Novus Biologicals, Littleton, US). As secondary antibodys, anti-mouse and anti-rabbit IgG alkaline phosphate conjugate (Sigma Aldrich, St. Louis, MO, USA) antibodies were applied, and signal visualization was done with Sigma Fast TM BCIP/NBT (Sigma Aldrich, St. Louis, MO, USA). $\alpha$-actin-specific antibody was provided as a loading control (1:2000, Sigma Aldrich, St. Louis, MO, USA).

\subsection{Gene specific silencing}

siRNA mediated transfection method was used to achieve gene-specific silencing. HPV-KER immortalized keratinocytes were transiently transfected by siRNA products at approximately $70 \%$ confluency. Reagents for silencing (medium and transfection reagent) were ordered from Santa Cruz Biotechnology (Dallas, TX, USA). In the experiments, scrambled and gene-specific LUC7L3, PPIG, SFRS18 siRNA duplexes were also applied. The most suitable effectiveness was achieved in serum-free culture medium, which does not contain additives and supplements. The silencing efficacy was controlled by real-time RT-PCR. 


\subsection{Polymerase chain reaction}

Total RNA collected from siRNA transfected HPV-KERs was isolated from the keratinocyte cultures by TRIzol ${ }^{\circledR}$ Reagent (Invitrogen Corp., Carlsbad, CA, USA), following the instructions provided in the manual. cDNA synthesis was accomplished from $1 \mu \mathrm{g}$ total RNA with the iScript cDNA Synthesis Kit (Bio-Rad Laboratories, Hercules, CA, USA). Human fibronectin specific primers were applied for the amplification of an $847 \mathrm{bp}$ product from the EDA-, and a 1221 bp product from the EDA+ fibronectin template. PCR reactions were carried out as described previously, and semiquantitative detection of the differentially spliced fibronectin isoforms are also detailed in this report [50]. To the analysis, $10 \mu \mathrm{l}$ of the PCR products were run on $1 \%$ agarose gel at $90 \mathrm{~V}$, and after that, the results were photographed and evaluated by Bio-Rad Gel Doc XR densitometer.

\section{$\underline{\text { 3.9 Flow cytometry }}$}

siRNA silenced HPV-KER keratinocytes were collected with careful trypsinization $(0.25 \%$ trypsin solution Sigma Aldrich, St. Louis, MO, USA), fixed in Fixation/Permeabilization Concentrate and Diluent (eBioscience, San Diego, USA) and resuspended in phosphatebuffered saline (PBS). The anti-EDA ${ }^{+}$-fibronectin and anti-fibronectin primary antibodies were used for $45 \mathrm{~min}$ at dilution 1:500 and 1:1000, respectively (both purchased from Sigma Aldrich, St. Louis, MO, USA). The PBS-washed keratinocytes were then incubated with the secondary antibody (anti-mouse IgG-Alexa Fluor 647, dilution of 1:500; Life Technologies, Carlsbad, CA). Fluorescent detection was executed on a FACSCalibur flow cytometer equipped with 488 and 633 nm lasers (Becton-Dickinson, Franklin Lakes, NJ, USA)

\subsection{Sequencing}

Sequencing was carried out on the Illumina HiScan SQ platform in the Center for Clinical Genomics and Personalized Medicine, University of Debrecen. Illumina-compatible ScriptSeq RNA-Seq Library Preparation Kit (Epicenter) was used for 2x100 bp paired-end library construction, by sequencing two technical replicates per condition ( 2 control and 2 siRNA- 
silenced samples derived from immortalized HPV-KERs). The average sequencing depth was $\sim 35$ million raw reads per sample.

\section{$\underline{\text { 3.11 Statistical analysis and bioinformatics }}$}

For the statistical analysis of the results from polymerase chain reactions, densitometry and flow-cytometry mesurements, GraphPad Prism 5.0 Software (GraphPad Prism, San Diego, CA) was used, using one-sample $t$ - and unpaired $t$-tests as necessary, with $\mathrm{p}<0.05$ as the threshold of statistical significance. Predictive interaction analyses were performed in the publicly available STRING database (http://string-db.org/). FASTQ format raw reads were submitted to quality control using FASTQC, followed by quality and adapter trimming using the fastx toolkit. The Hg19 human genome reference was used for mapping filtered reads with the STAR aligner, using parameters optimized for splice-site discovery [75]. Subsequently, alignment to potential contaminant sequences and the HPV genome were carried out, and ambiguously mapping reads were discarded from further analysis. TopHat 2 was used for potential fusion transcript discovery using the fusion-search algorithm [76]. Transcript assembly and annotation were carried out using Cufflinks (Trapnell et al., 2010) after mapping, and read counts were summarized at the gene and exon levels by using htseq-count [78]. Count data were then processed using the DESeq and DEXSeq packages to quantify differential gene expression and differential exon usage, respectively [79]. Exons with a coverage $<5$ reads were discarded from the analysis. Functional annotation of differentially expressed genes was carried out using Gene Ontology (GO) enrichment, and extensive comparisons with previous literature-based gene sets and data visualization were carried out in $\mathrm{R}$ version 3.0.1, and Cytoscape. 


\section{RESULTS}

\subsection{SR-rich splicing factor genes exert decreased response to T-lymphokine stimuli in} psoriatic non-involved epidermis as compared to healthy samples

In the course of our previous cDNA microarray experiment, 61 previously annotated genes and additional 11 transcipts were found to be differentially regulated in psoriasis upon $\mathrm{T}$ lymphokine treatment [71]. The subsequent Gene Ontology Analysis revealed three splicing regulators among the identified genes: luc-7 like protein 3 (LUC7L3), peptidyl-prolyl cis-trans isomerase G (PPIG) and arginine/serine-rich 18 (SFRS18). Real-time RT-PCR was performed to control whether the identified genes showed an altered responsiveness due to T-lymphokine induction. The lymphokine-stimulated changes were verified in the case of both LUC7L3 and PPIG: while their expression is upregulated in healthy epidermis, no changes or even downregulation were observable in psoriatic non-involved epidermis (Fig. 5.) Although we were unable to validate the microarray results for SFRS18, we included this gene in the further studies, because it is involved in similar biological processes as LUC7L3 and PPIG.
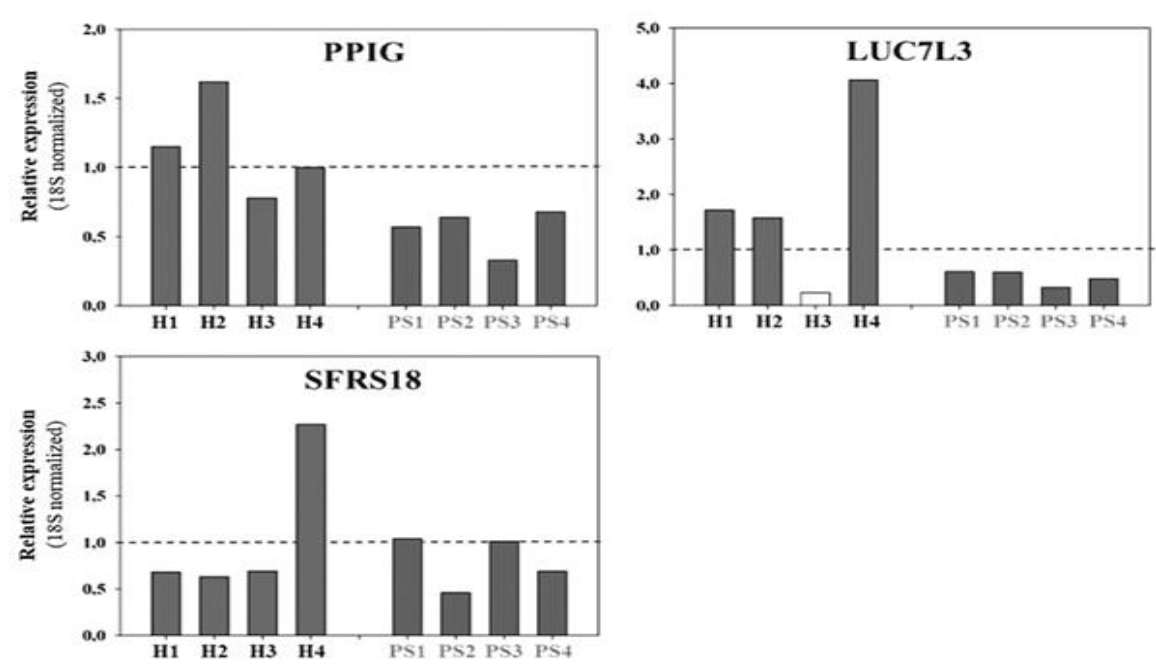

Fig. 5. T-lymphokine induced altered gene expression of splicing factors was compared in healthy $(\mathrm{H}, \mathrm{n}=4)$ and psoriatic non-involved (PS, $\mathrm{n}=4)$ epidermis. As baseline, the average expression of untreated samples was considered (relative unit of 1 indicated by dashed line). 
In addition, we decided to compare the mRNA expression levels of the splicing regulators in untreated healthy and psoriatic non-involved skin specimens of the cDNA microarray experiment: in this manner of comparison, the expression of LUC7L3 and SFRS18 was found to be slightly higher in psoriatic non-involved samples than in healthy epidermis, while PPIG did not exhibit any alterations (Fig. 6.).
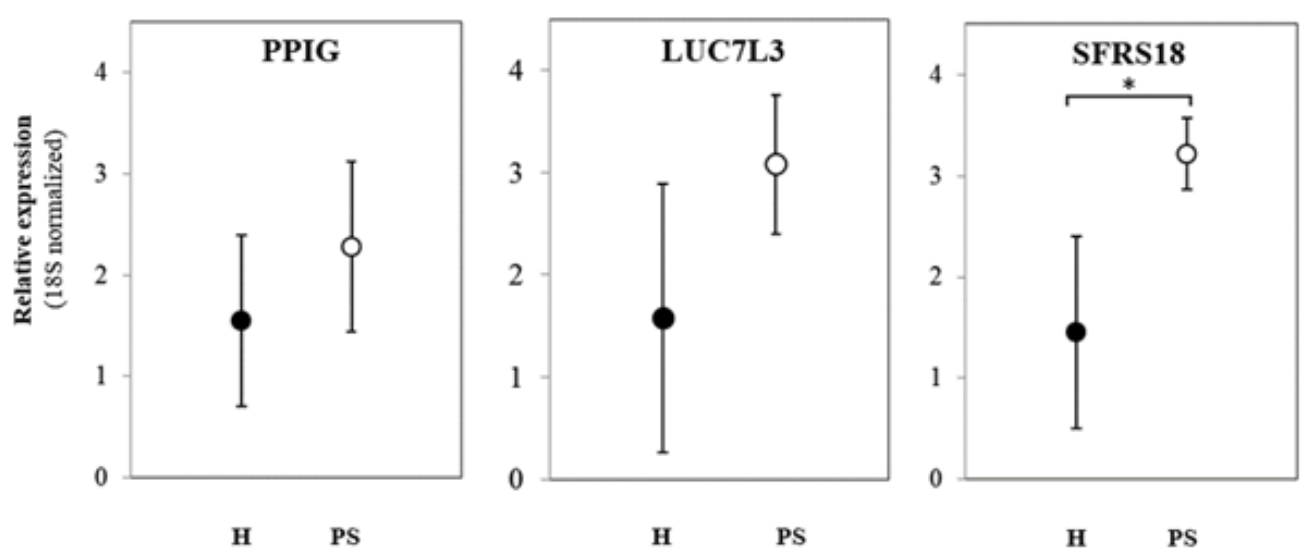

Fig. 6. LUC7L3, PPIG and SFRS 18 basal mRNA expression in untreated healthy $(H, n=4)$ and psoriatic non-involved (PS, $\mathrm{n}=4$ ) samples. $\mathrm{P}<0.05$ was considered as significance treshold

\subsection{LUC7L3, PPIG and SFRS18 show altered expression in psoriasis.}

Since LUC7L3, PPIG and SFRS are poorly characterized, we investigated their protein expression pattern among healthy, psoriatic non-involved and involved skin samples. LUC7L3 and PPIG exerted a nuclear localization while staining for SFRS18 was rather perinuclear (Fig. 7. a). LUC7L3 could be detected in significantly higher amounts in psoriatic non-involved epidermis, whereas SFRS18 presents only minimal, non-significant upregulation in noninvolved epidermis. Both LUC7L3 and SFRS18 exhibit the highest levels of expression in psoriatic involved epidermis. Pattern of expression for PPIG was distinct from that of the other splicing regulators, because PPIG exerts a significant decrease in psoriatic non-involved epidermis. However, the highest amount of LUC7L3, PPIG and SFRS18 was detected in psoriatic involved epidermis (Fig. 7. b.). 

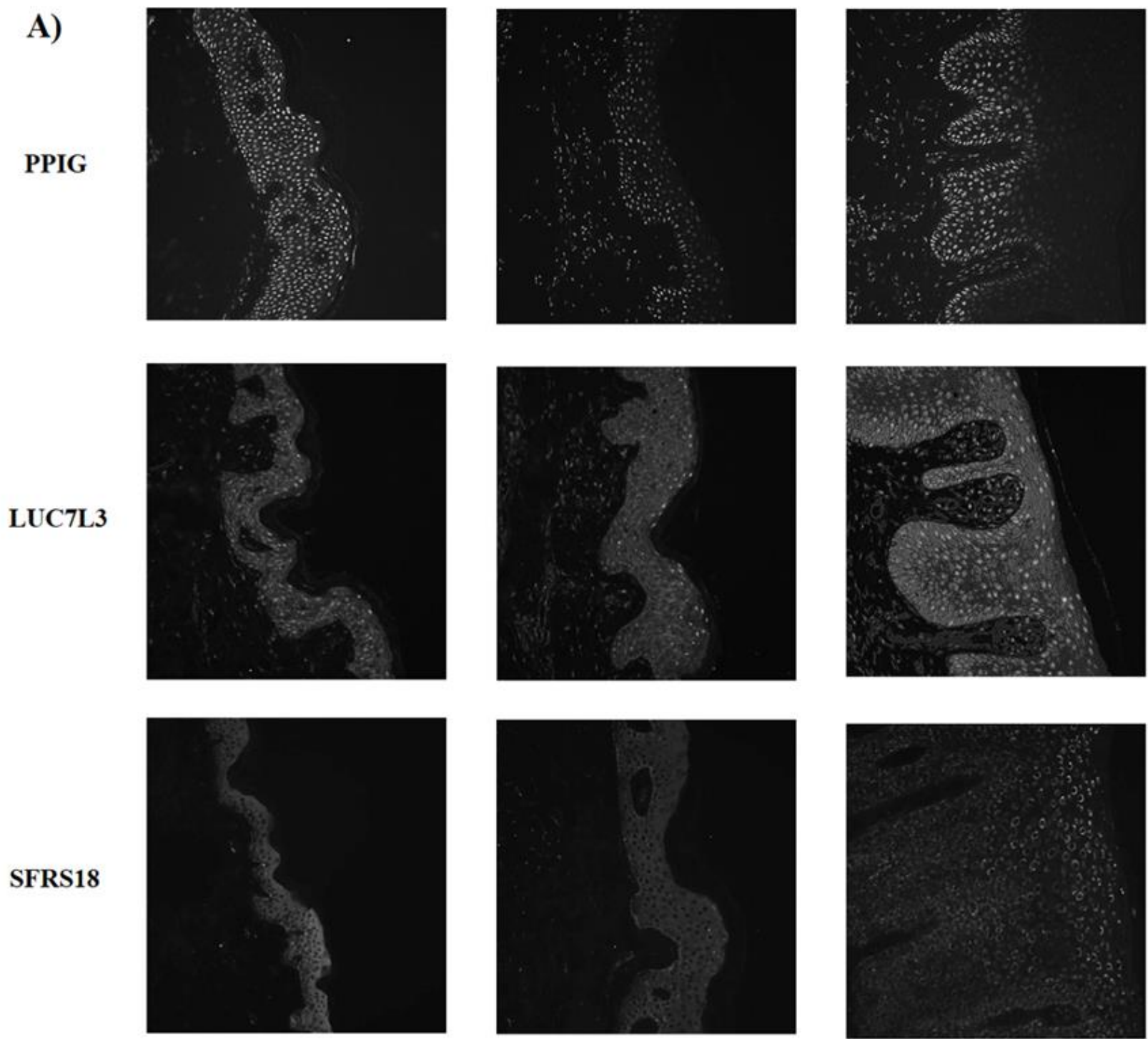

Healthy epidermis

Psoriatic non-involved epidermis

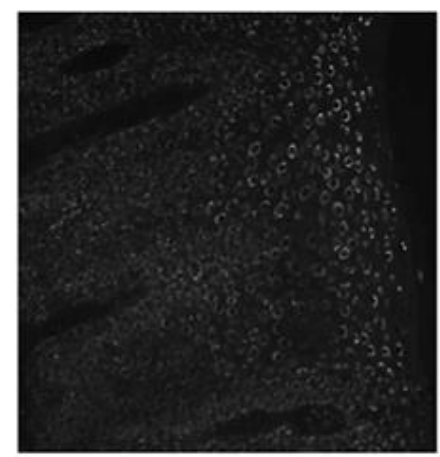

Psoriatic involved epidermis

B)
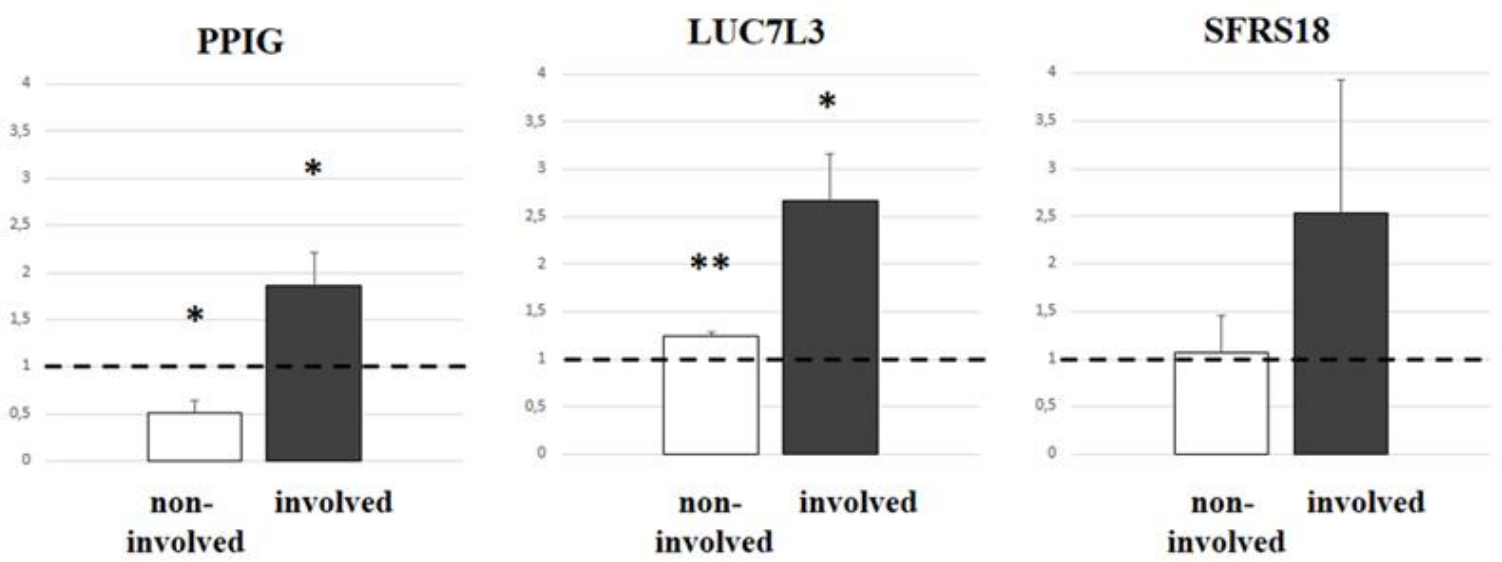
Fig. 7. Immunofluorescent staining of PPIG, LUC7L3 and SFRS18 proteins (a) were performed on healthy, psoriatic non-involved and involved epidermis ( $n=3$, in all cases). For each protein, a representative staining is introduced. Magnification: 40x. Fluorescent intensity detection of tissue stainings were also performed (b) and non-involved and involved samples were compared to healthy specimens: dashed lines indicates the unaltered condition, where the ratio of non-involved/healthy and involved/healthy is 1 ; significance levels are represented by asterisks $(\mathrm{p}<0.001 * * *, \mathrm{p}<0.01 * *, \mathrm{p}<0.05 *)$

\subsection{LUC7L3, PPIG and SFRS18 exhibit similar pattern of expression in synchronized,} immortalized keratinocytes

As the further step of the study, we aimed to determine the expression features of LUC7L3, PPIG and SFRS18 during keratinocyte proliferation and differentiation. Both mRNA and protein expression patterns were characterized in synchronized, immortalized cell lines. To this end, HPV-KER cells were synchronized via contact inhibition and withdrawal of growth factors. After release from cell quiescence, HPV-KER cells were harvested at various time points, and we applied real-time RT-PCR and western blot analysis to assess RNA and protein levels of splicing factors.

During comparison of splicing factor expression in different states of the proliferation and differentiation, we observed a very similar mRNA expression pattern for LUC7L3, PPIG and SFRS18 (Fig. 8.a). After a transient mRNA abundance decrease around $12 \mathrm{~h}$, a peak of mRNA expression was found at $24 \mathrm{~h}$, accompanied with a steady elevation, which started from $48 \mathrm{~h}$.

Similarly to the mRNA level changes, the protein level expression changes were very similar during the proliferation-differentiation cycle of HPV-KER cells. However, protein levels were proven to be more even compared to mRNA level changes. The highest amount of splicing regulator proteins was displayed at $48 \mathrm{~h}$ after release from cell quiescence (Fig. 8.b).

Moreover, the synchronization experiment was repeated in another type of immortalized keratinocyte: the synchronized HaCaT cells - similarly to the results obtained in HPV-KERs express splicing factors with an identical pattern (Fig. 9.). 

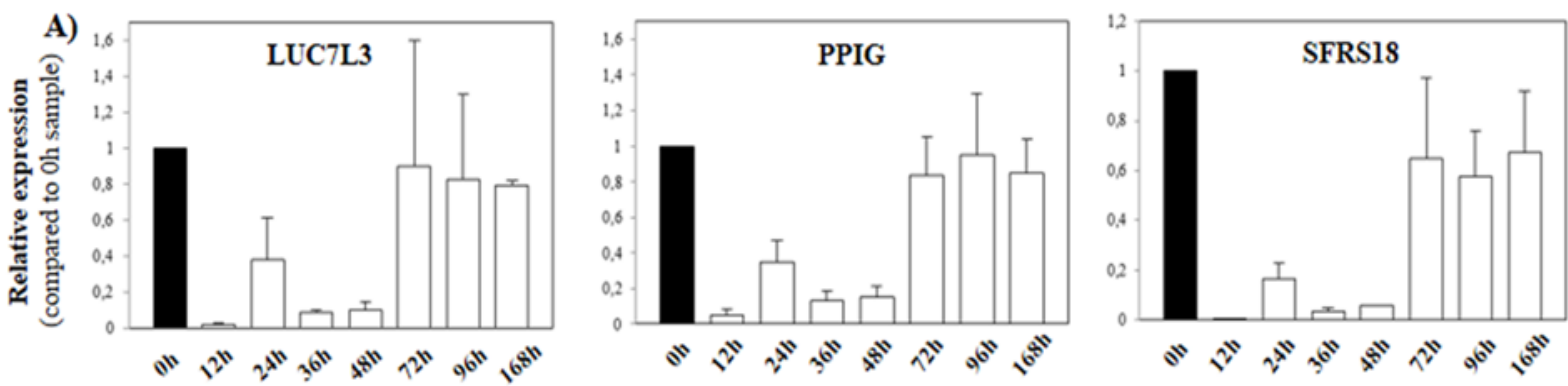

B)
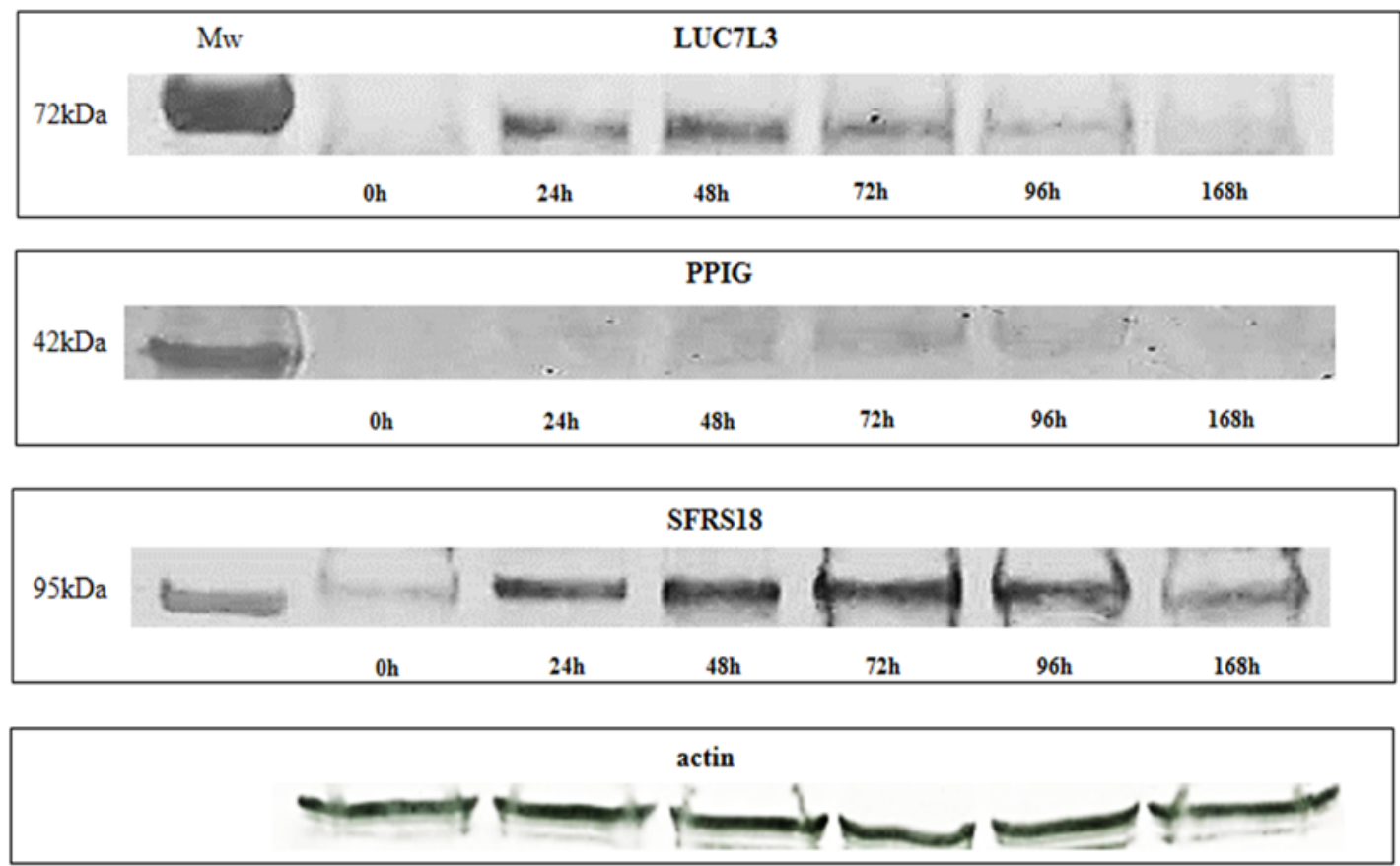

Fig. 8. Synchronization of HPV-KER cells was achieved via contact inhibition and supplement withdrawal, and sample collection from the re-passaged $(0 \mathrm{~h})$ cells was done at the indicated time points. Alterations in mRNA abundance (a) were measured by real-time RT-PCR, regarding protein levels (b) western blot analysis was used for determination. The experiments were performed with three independent series of cell cultures, and the mRNA amounts were calculated as the average of the parallels. For each splicing factor, one representative picture of western blot is provided. $\alpha$-actin served as a loading control. 

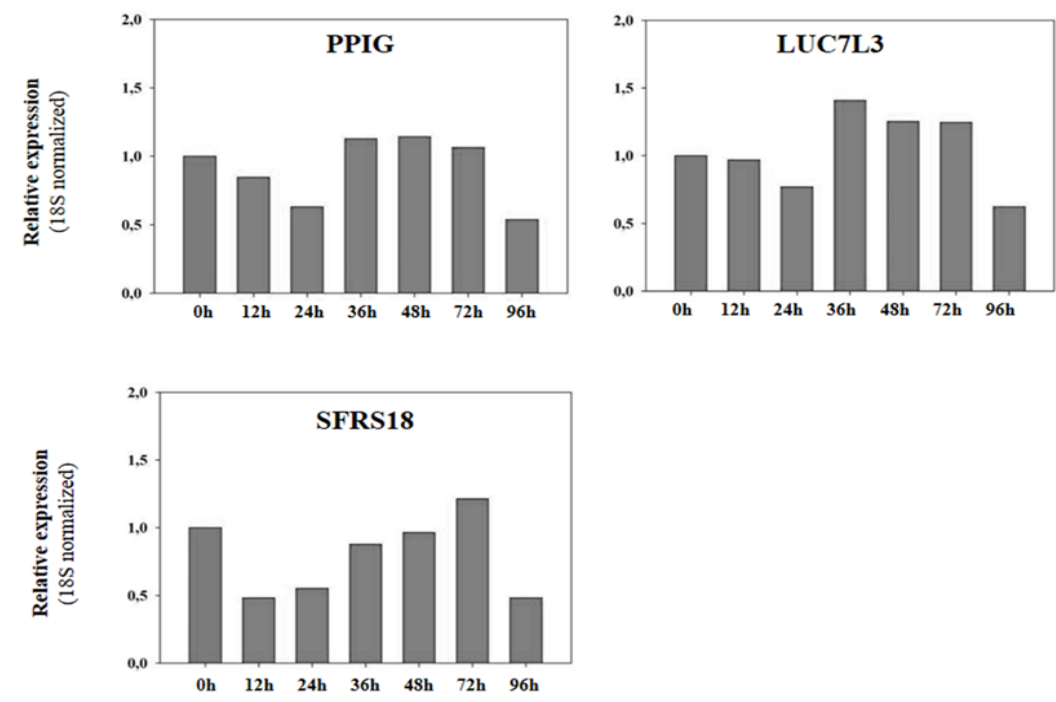

Fig. 9. Detection of LUC7L3, PPIG and SFRS18 mRNA changes in synchronized, immortalized HaCaT cells $(\mathrm{n}=1)$. Synchronization was executed according to Pivarcsi et al., and samples were taken at the indicated time points following the re-passage $(0 \mathrm{~h})$ (Pivarcsi et al., 2001). Considering the mRNA expression, the three splicing regulators exert identical expression patterns after the release from cell quiescence.

\section{4 siRNA silencing of $P P I G, S F R S 18$ and $L U C 7 L 3$ gene expression alter the ratio of $\underline{\text { EDA+/total fibronectin }}$}

After the analysis of $L U C 7 L 3, P P I G$ and SFRS18 gene expression characteristics, we decided to explore molecular mechanisms influenced by these splicing regulators. As former results of the research group suggested that certain fibronectin splicing abnormalities might possess pathogenic role in psoriasis, first, we aimed to assess the relevance of LUC7L3, PPIG and SFRS18 in the mRNA maturation processes of this protein. Therefore, HPV-KER cells were submitted to siRNA-mediated silencing of the splicing factor genes. The EDA+/total fibronectin ratios were compared in scrambled and specifically transfected cells, the effect of the silencing was high, with an average of approximately $80 \%$. Alterations in the fibronectin splicing pattern were detected by RT-PCR, and densitometry was employed as a semiquantitive technique to investigate the EDA+/total fibronectin ratio changes upon silencing of the three 
splicing factors. During the course of the experiments either single or combined silencing was performed, in order to observe possible interactions among the LUC7L3, PPIG and SFRS18 splicing factors.

As other types of undifferentiated cells, HPV-KERs contain higher amount of the EDA+ fibronectin isoform than the EDA- variant. In single silencing experiments, siRNA transfection of LUC7L3 diminished the relative amount of the EDA+ isoform, and a slight decline in this ratio was also also demonstrated with PPIG and SFRS18 silencing (Fig. 10.a).

Bioinformatics prediction using the STRING database suggests that LUC7L3 and SFRS18 are presumably interactors (Fig. 10.c). As mentioned above, to examine the interference of splicing factors, simultaneous silencing of two or three genes was executed. In accordance with the predictions, the most robust alteration in the fibronectin EDA+/total ratio was achieved by the combined silencing of LUC7L3 and SFRS18 (Fig 10.a). Furthermore, triple silencing does not exert additional effect on the ratio compared to the combined silencing of LUC7L3 and SFRS18. The consequences of silencing were also evaluated at the protein level. The EDA+ and total fibronectin levels were analyzed by flow cytometry using the most robust silencing combination determined in the course of the previous experiments. In agreement with the gene expression experiments, the EDA+ fibronectin isoform was present in higher amounts in the immortalized keratinocytes as compared to the EDA- variant. The double silencing of $L U C 7 L 3$ and SFRS18 significantly decreased the quantity of the EDA+ isoform without changing the amount of total fibronectin (Fig. 10.b). In conclusion, these data indicate that the decresed levels of the splicing regulators result in diminished EDA+/total fibronectin ratio both at mRNA and protein levels. 

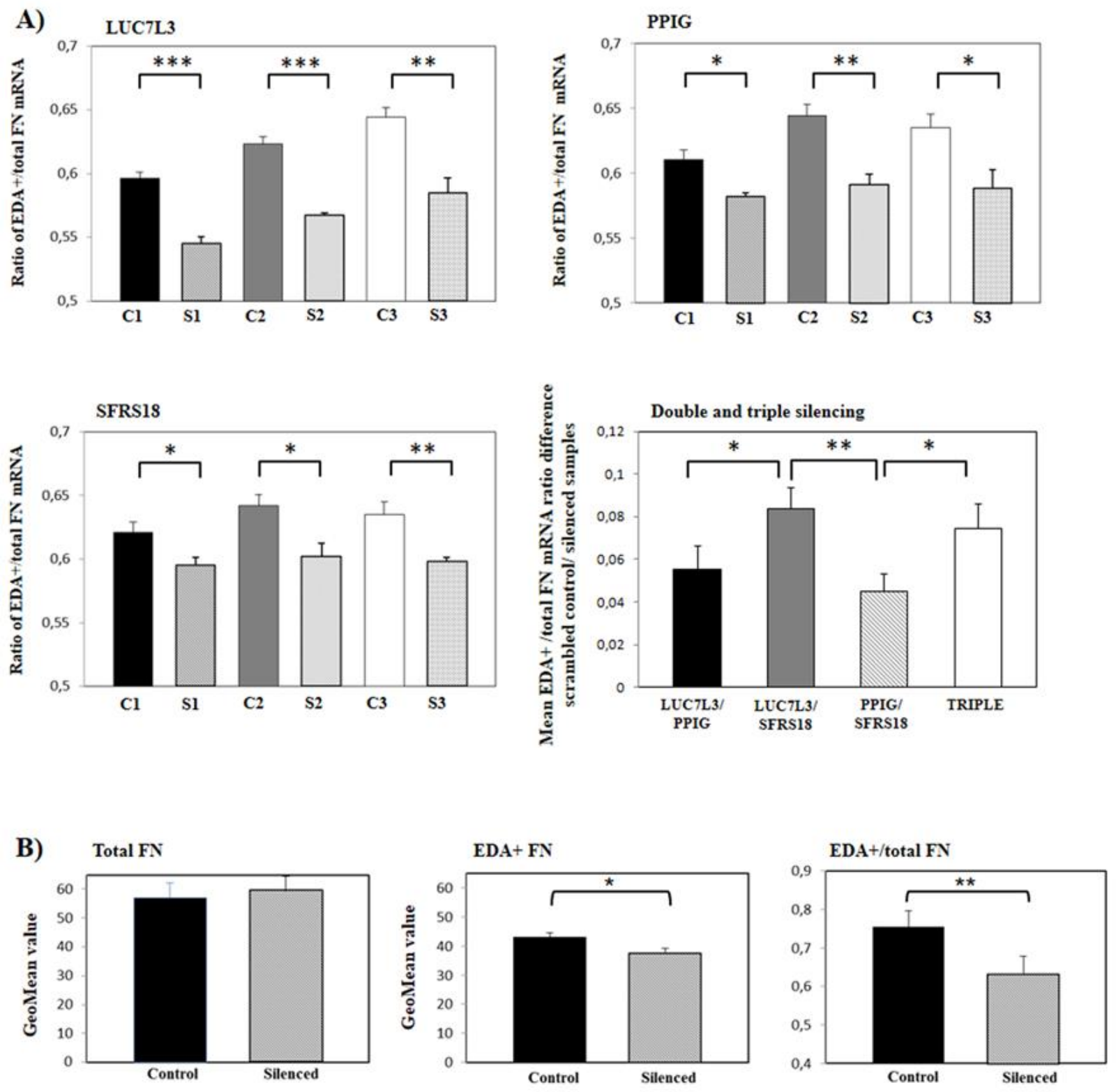

C)
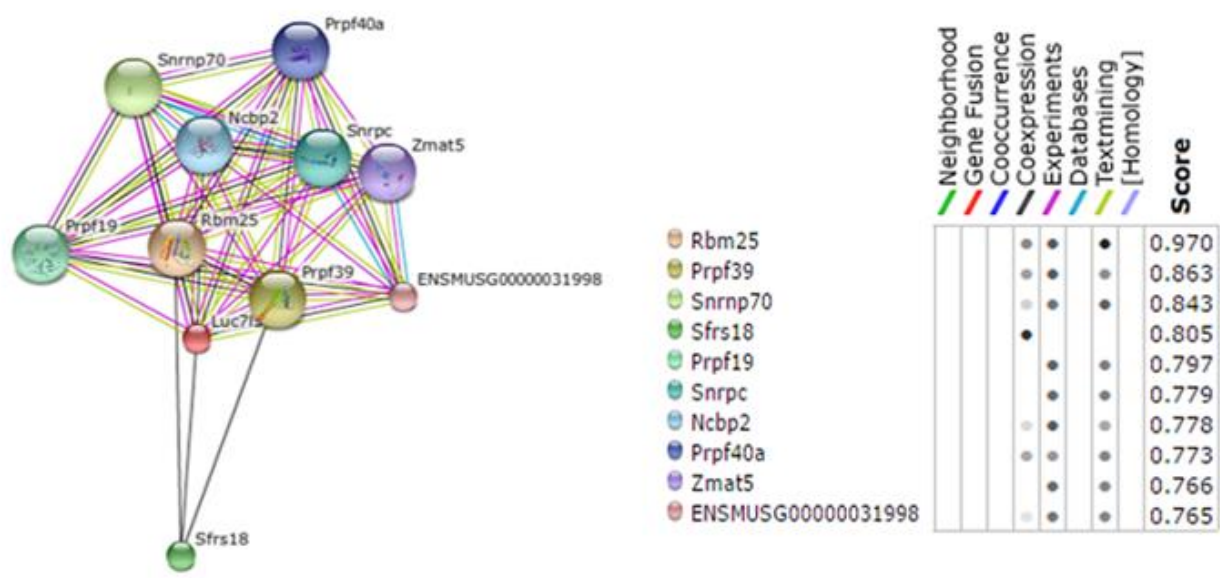
Fig. 10. HPV-KER cells were transfected with scrambled and specific siRNA constructs targeting LUC7L3, PPIG and SFRS18 (a) and the EDA+/total fibronectin ratio was detected in each series (S1, S2, S3). The changes in this ratio were compared in each experiment to that of the scrambled RNA transfected controls $(\mathrm{C} 1, \mathrm{C} 2, \mathrm{C} 3)$. Effect of double and triple silencing was also demonstrated. Columns represent means (+S.E.) of three independent experiments, which are all performed in triplicate and significance levels are marked by asterisks $(\mathrm{p}<0.001 * * *$, $\mathrm{p}<0.01 * *, \mathrm{p}<0.05 *)$

Total fibronectin and EDA+ fibronectin were determinated by flow cytometry in LUC7L3/SFRS18 double silenced HPV-KERs (n=5) (b). Silencing had not any affect on the quantity of total fibronectin, while a significant $(\mathrm{p}<0.05)$ EDA+ decrease was detected upon silencing, also resulted a significant $(\mathrm{p}<0.01)$ changes in the EDA+/total fibronectin ratio. Bioinformatics prediction suggested a possible interplay between the LUC7L3 and SFRS18 regulators (c)

\subsection{Global transcriptome analysis of immortalized keratinocytes}

\subsubsection{Determination of silencing efficacies and quality control}

In our further experiments, we intended to analyze other additional targets of LUC7L3, PPIG and SFRS18 in order to identify biological patways affected by these regulators. For the subsequent experiments, we have chosen the dual silencing of LUC7L3/SFRS18, which proved to be most robust in the generation of splicing pattern alterations of fibronectin. This experimental design enables a powerful methodological approach, therefore we carried out RNA-Seq profiling of immortalized HPV-KER cells, which is also capable of the detection of distinct splice variants and non-coding transcripts, as well. Silencing efficiencies were screened via Real-Time RT-PCR prior to sequencing, which suggested a suitable silencing efficacy, between $\sim 70-80 \%$ (Fig. 11.). Quality control of silenced sample isolates was performed by Bioanalyzer and indicated excellent quality (RIN: 10) (Fig. 12.). 


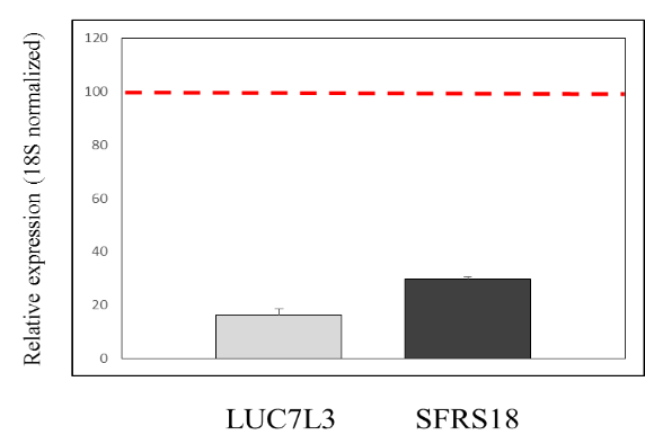

Fig. 11. Double silencing of $L U C 7 L 3 / S F R S 18$ was found to be the most effective in modifying fibronectin mRNA maturation. The silencing efficacy was found to be sufficient $83.6 \% \pm 4,24$ for $L U C L 73$ and $70,1 \% \pm 1,56$ for SFRS18, in two replicates
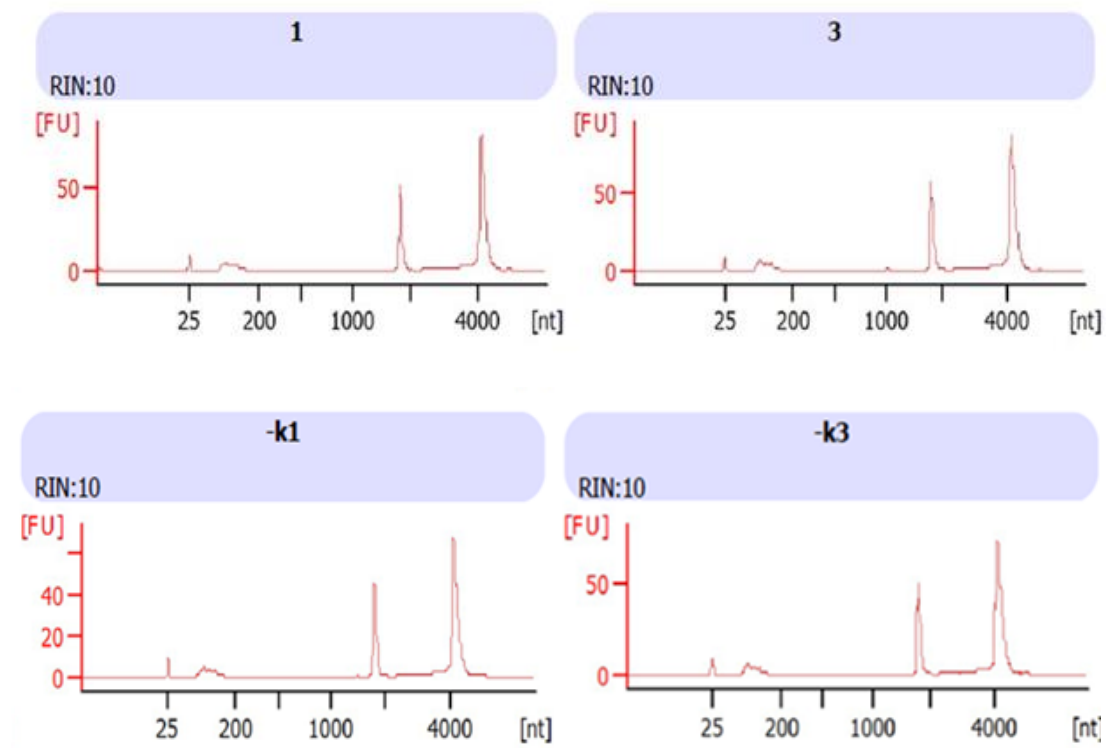

Fig. 12. Quality control of $L U C 7 L 3 / S F R S 18$ siRNA transfected samples $(1,3)$ and scrambled controls (-k1, -k3).

\subsubsection{Paired-end RNA-Sequencing reveals differential expression and exon usage patterns upon the silencing of the LUC7L3 and SFRS18 splicing factors}

Sequencing reads of $2 \times 100$ bp were mapped to the Hg19 human reference genome, followed by de novo transcript assembly, in order to identify transcript isoforms expressed in the HPV- 
KER cell line, and to provide accurate exon-level annotations for downstream analyses. Differential exon usage and differential expression metrics were generated separately, to assess the alterations caused by the dual silencing of splicing factors. Differential exon usage was analysed instead of differential isoform expression in order to circumvent the complexity, and possible errors introduced by isoform reconstruction methods [80, 81].

$\mathbf{A}$

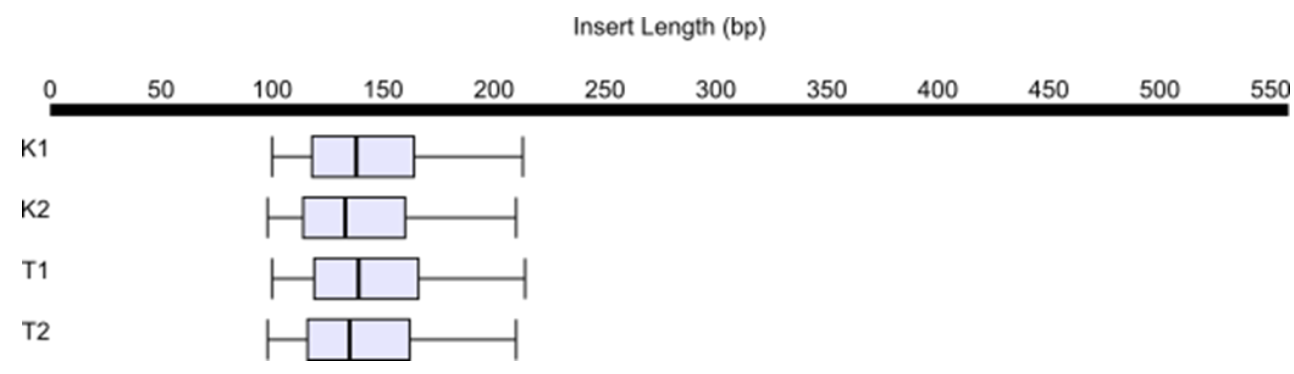

B

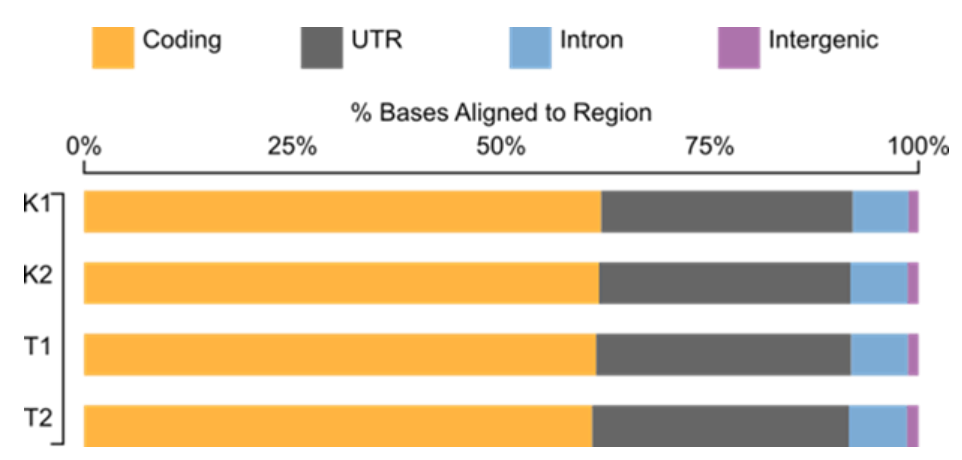

Fig. 13. Box-plot of insert length distributions in the 2x100 bp RNA-Seq library (a). Relative percentages of read coverage per transcript type in the alignments (b).

\subsubsection{Differential gene expression}

The combined silencing of the LUC7L3 and SFRS18 resulted in moderate changes in gene expression, however extensive disturbances in exon usage. This finding was in accordance with expectations based on the experimental model. Thirty-five protein-coding genes were identified as differentially expressed ones $(\operatorname{logFC}>0.5$, FDR $<0.05)$, with IFI6 and MX1, ISG15 and KRT6A mRNAs showing the most robust fold-changes. Regarding the functional enrichment 
analysis of significantly differentially expressed genes, the majority of LUC7L3 and SFRS18 regulated genes share functions related to Type I interferon signalling. The most significant enriched GO terms were cellular response to type I interferon (GO:0071357), cellular response to cytokine stimulus (GO:0071345), regulation of viral genome replication (GO:0045069); the functional relationships of significantly differentially expressed genes are illustrated in a functional interaction network based on the Reactome and GeneMania databases (Fig. 14.).

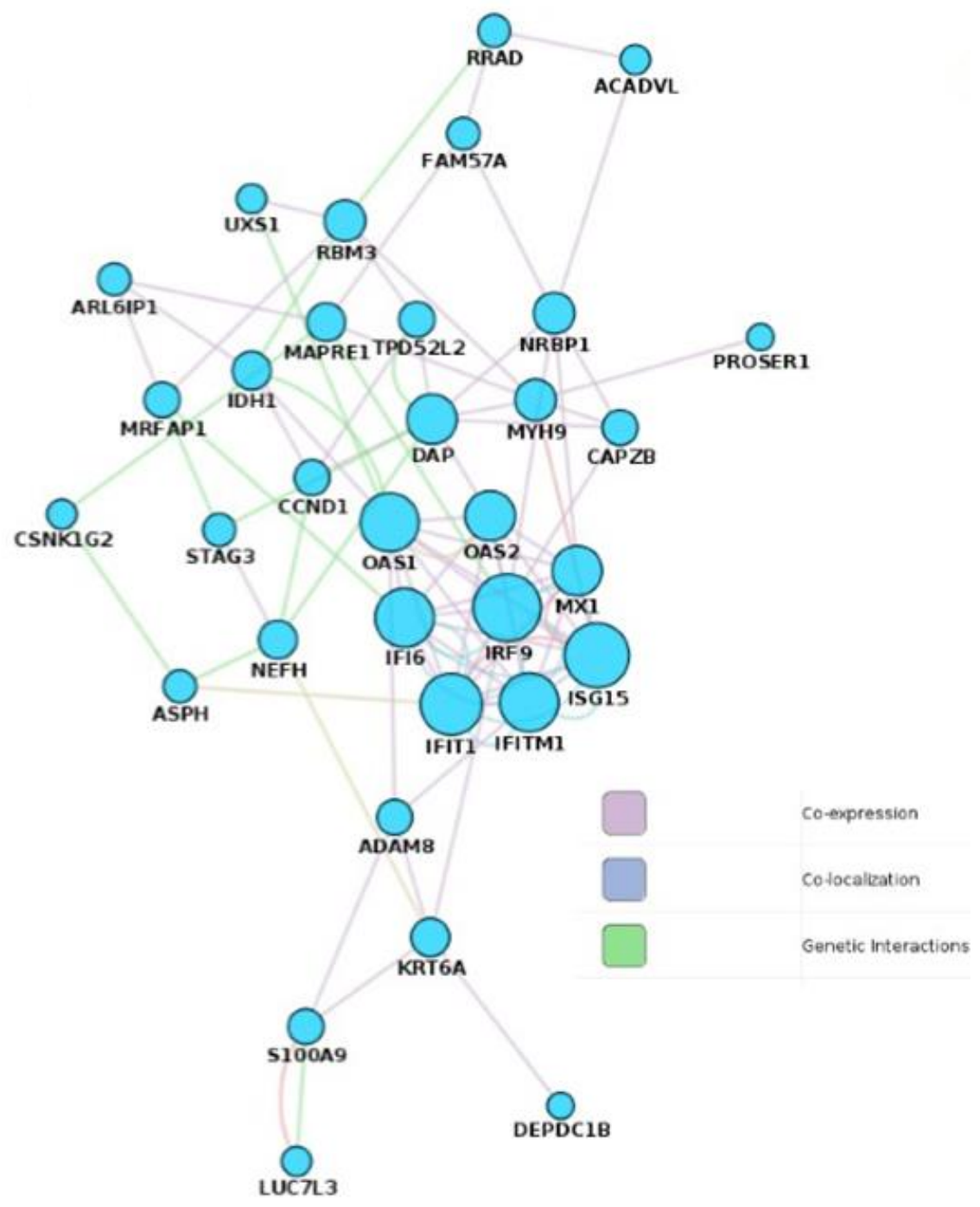

Fig. 14. Functional network of significantly differentially expressed genes $(\log 2 \mathrm{FC}>0.5$, FDR $<0.05$ ) in response to dual silencing of $L U C 7 L 3$ and SFRS18, with edges corresponding to GeneMania and Reactome annotation categories, and nodes corresponding to significantly regulated genes. 
According to the Real-Time RT-PCR validation, IFI6 displayed up to 4-fold upregulation, in agreement with the high-throughput results. Altogether three biological replicates (with double technical replicates of the RNA-seq experiment and double technical replicates of two prior LUC7L3/SFRS18 silencing experiments) were used for validation. An integrated figure of the three independent measurements is presented in Fig. 15.

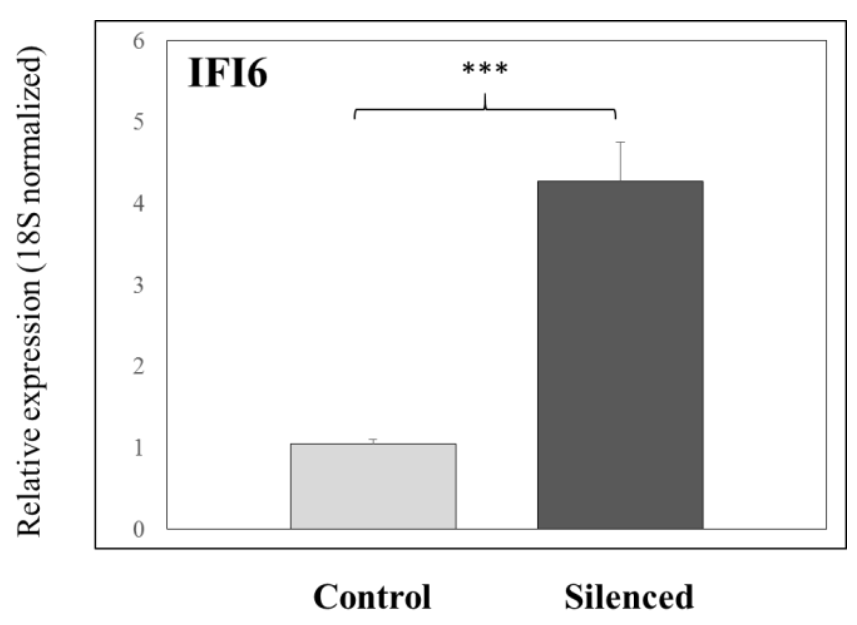

Fig. 15. Real-time RT-PCR validation of IFI6 mRNA expression changes. Columns indicate the mean of three independent experiments, with a significance threshold of $p<0.05$.

\subsubsection{Differential exon usage}

Following de novo transcript assembly, the extent of differential exon usage was measured, and indicated changes in 224 exons of 217 genes at a significance cut-off level of $\operatorname{logFC}>0.5$, FDR $<0.1$. The differential usage of multiple exons per gene is also shown in a considerable amount of genes, possibly indicating fine-tuned transcriptional changes induced by silencing (Fig. 16.). Differential exon usage was detected in several non-coding RNAs. Coordinated transcriptional regulation could be identified by mining co-expression-level databases. Functional enrichment analysis of the most significant genes shows an extensive co-expression, suggesting the possibility of overlapping regulation at transcriptional and/or posttranscriptional levels, and less pronounced enrichment of molecular functions (Fig. 17.). 
CREB1

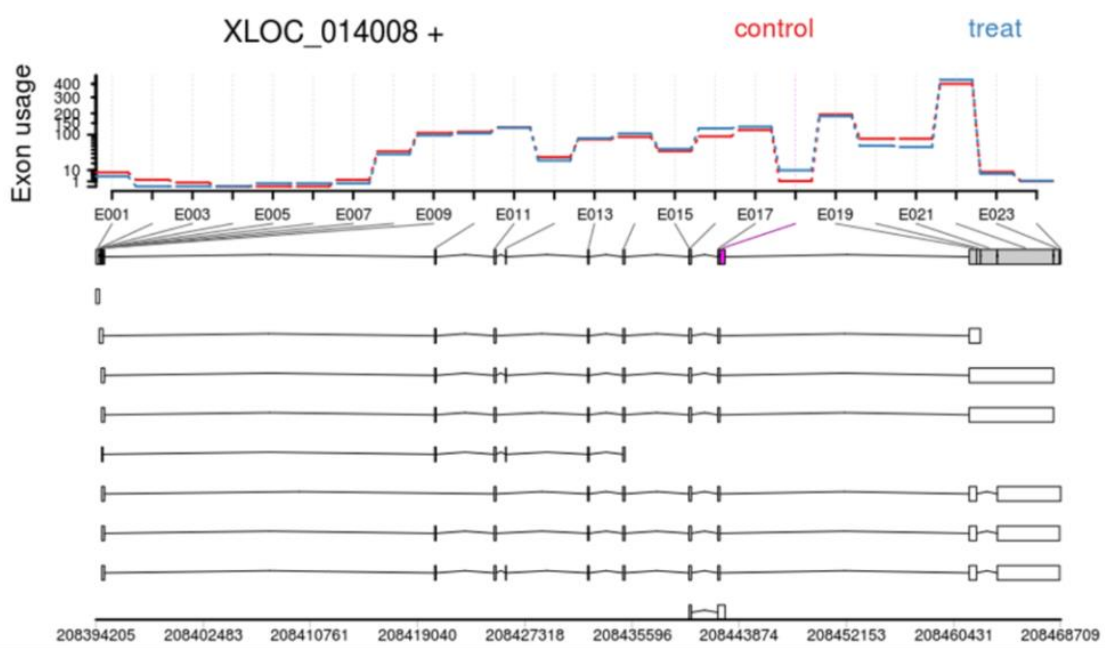

OAS2

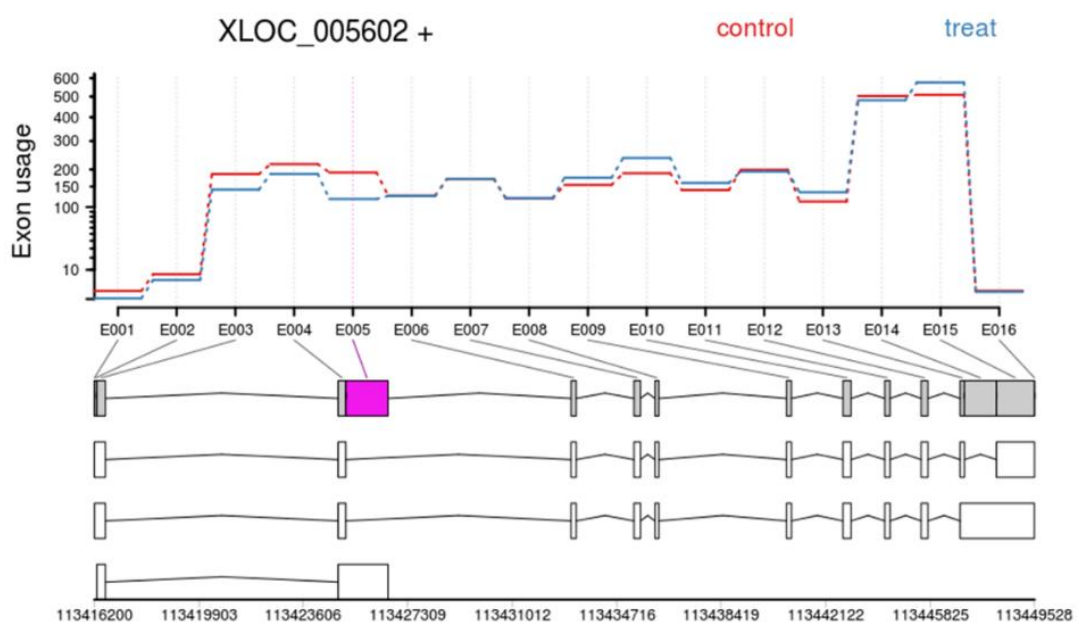

HERC6

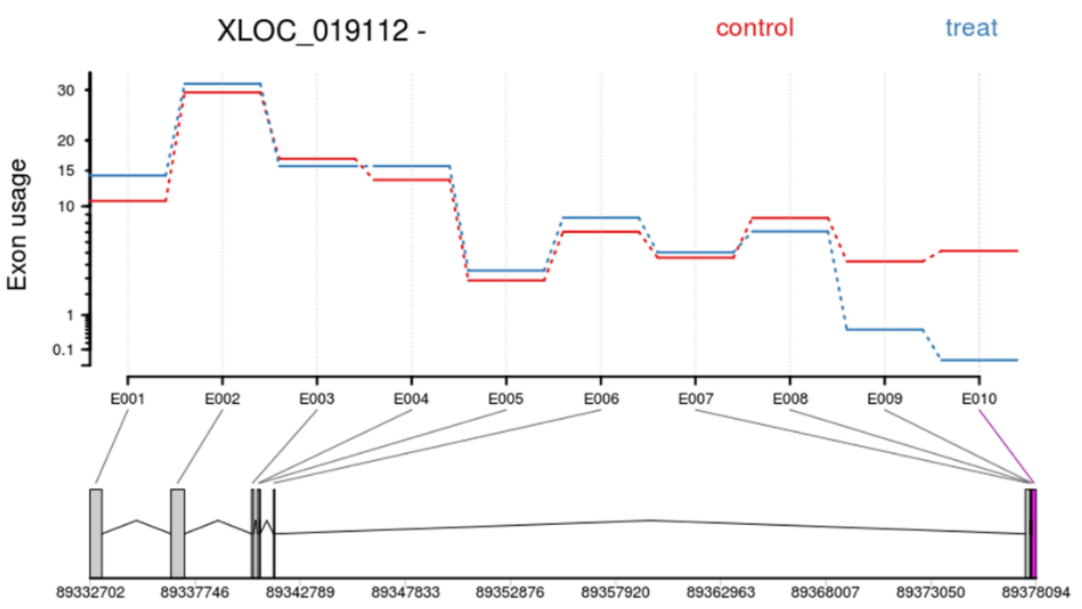


CUL1

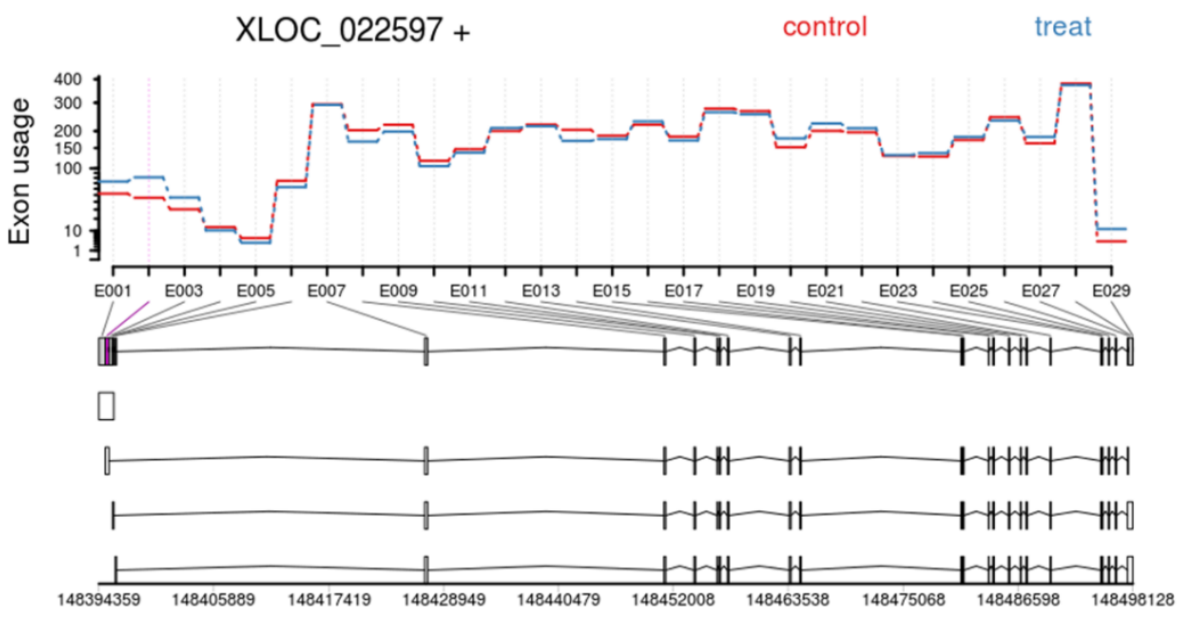

NEAT1

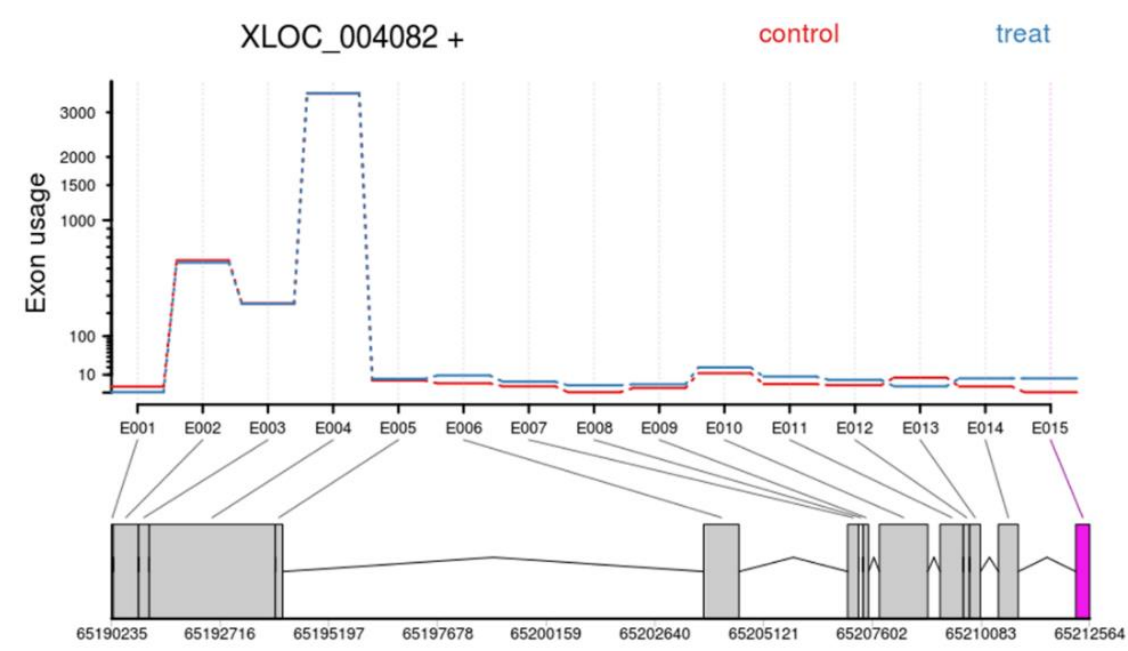

TINCR

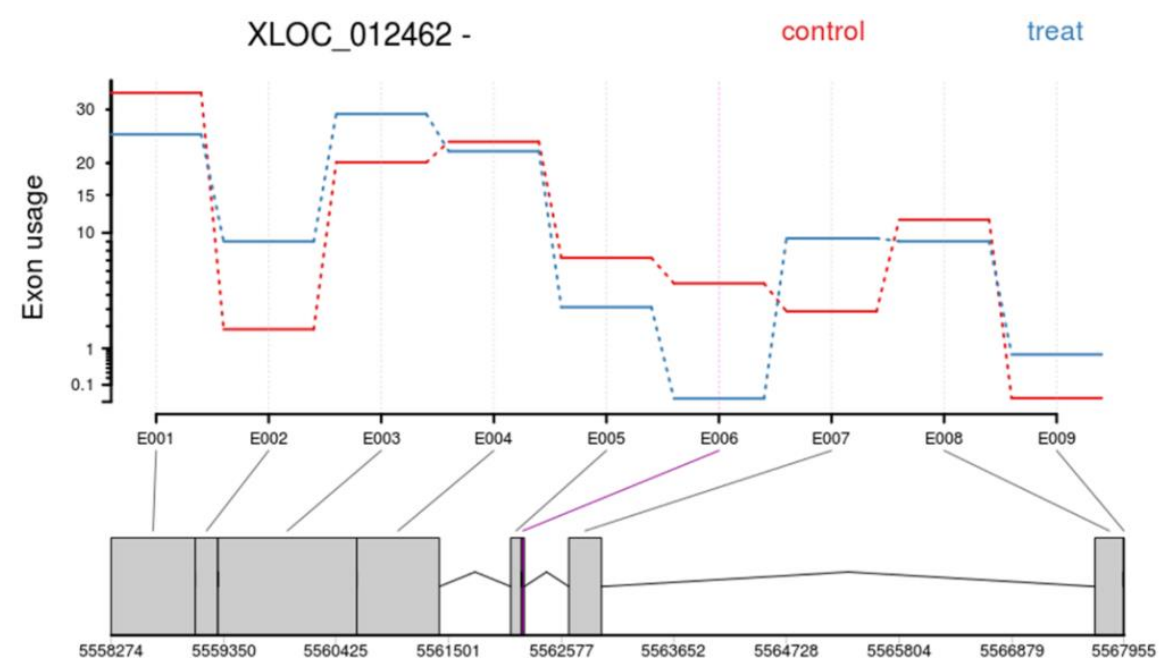


FN1

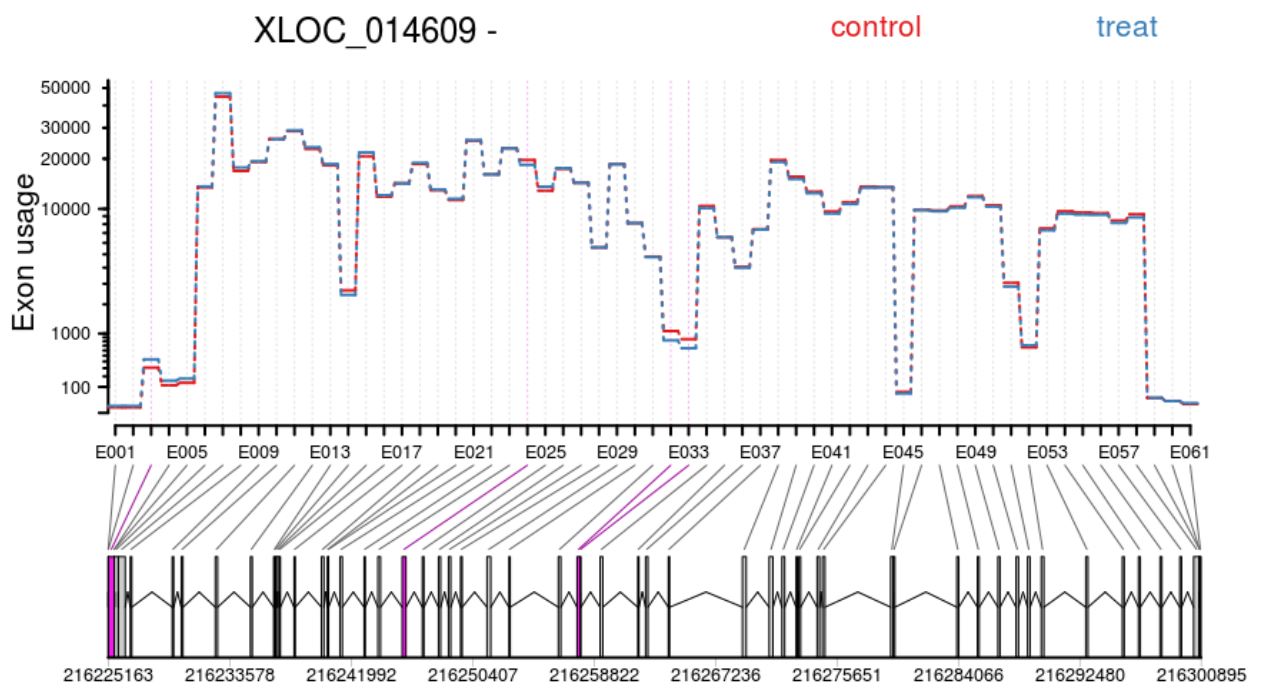

XLOC_013997 +

control

treat

NBEAL1

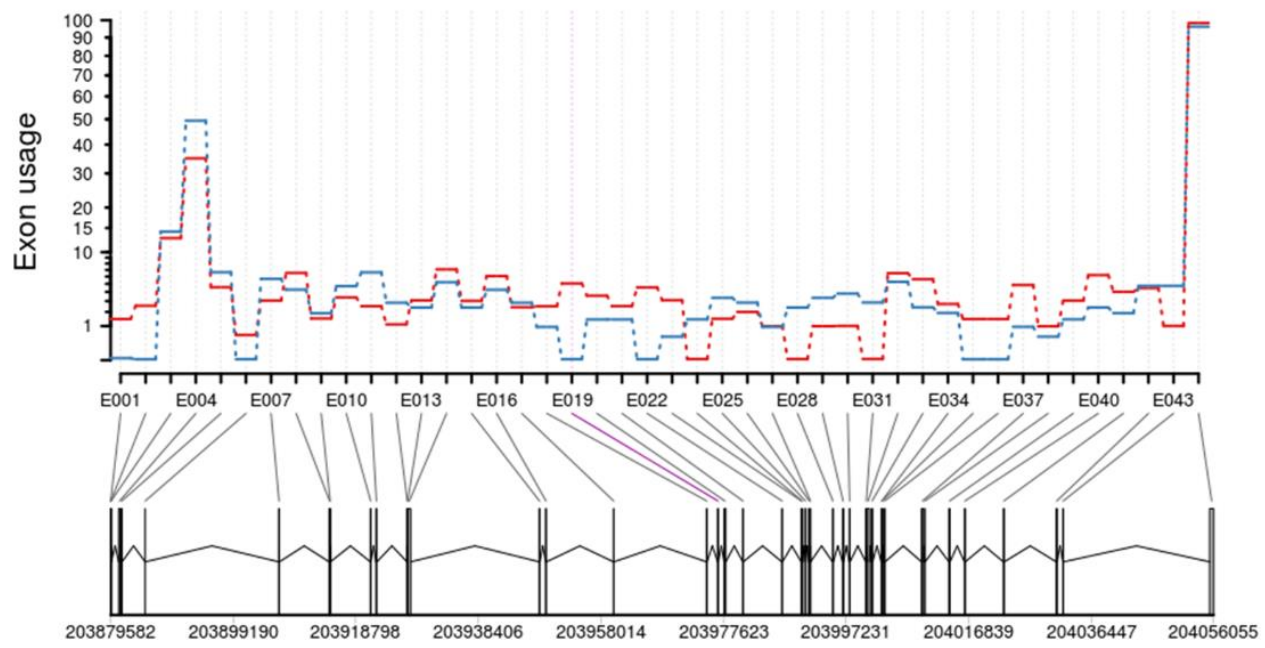

Fig. 16. DEXSeq differential-exon-usage plots of selected transcripts show significant alterations $(\log F C>0.5$, FDR $<0.1)$. Red: average exon usage frequency in control samples; blue: average exon usage frequency in treated samples; pink: significantly differentially expressed exons. De novo assembled transcript diagrams are depicted in bottom rows. 
Based on these results, a functional network of genes showing significant exon usage alterations was generated. The resulting functional network of transcripts displaying differential exon usage contains 172 genes, represented by nodes, with an average connectivity degree of 13.4, indicating high connectivity of the network. The majority of connecting edges between genes correspond to co-expression relations, followed by physical and genetic interactions. As the complete network is difficult to visualize, a smaller sub-network showing the most highly connected genes is illustrated in Fig. 17.

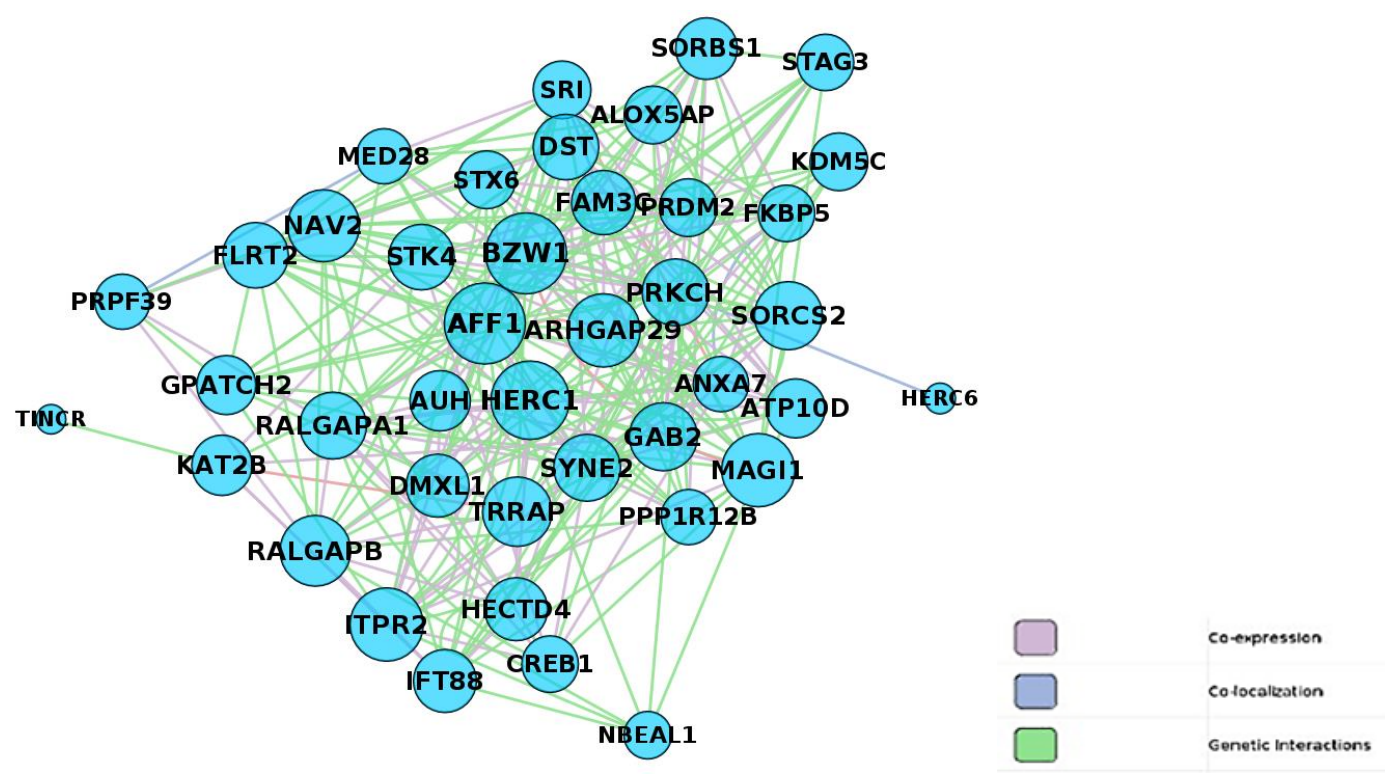

Fig. 17. Sub-network representation of a functional network constructed from genes exhibiting significant exon usage changes, with edge color corresponding to GeneMania and Reactome annotation categories.

Independent validations using RT-PCR were carried out for the exon inclusion/exclusion of fibronectin (FN1). The use of exon-specific, junction spanning primers for FN1 transcripts, followed by agarose gel electrophoresis, resulted in the detection of altered exon inclusion, and the decreased relative abundance of the EDA+ isoform, respectively (Fig. 18). 


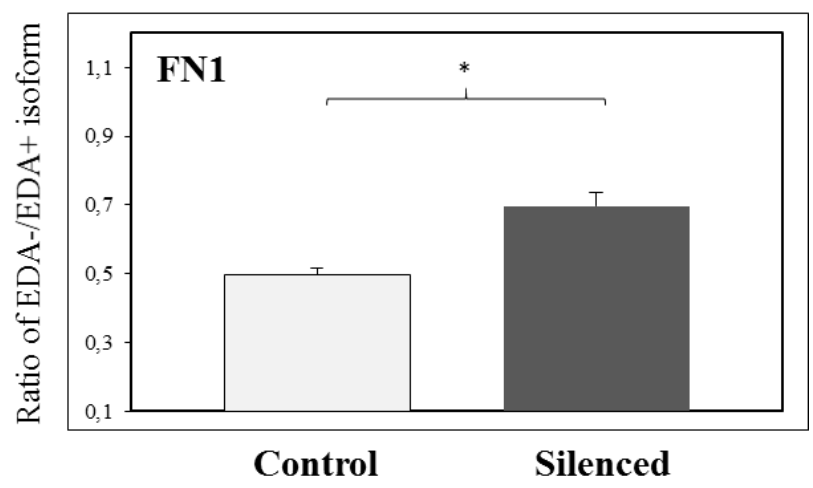

Fig. 18. Independent RT-PCR-based validation of differential exon usage, indicating abundance of alternative splicing variants in control (grey) and treated (black) samples in two replicates. 


\section{DISCUSSION}

Widespread application of large-scale gene expression studies proved to be fruitful in psoriasis research: the methodological developments provided a closer insight into disorder pathogenesis and facilitated the discovery of novel therapeutical targets [52, 53, 54]. In our recently conducted cDNA microarray experiment, we have focused on the early steps of psoriasis pathogenesis, which remain still only partially understood. In a cDNA microarray analysis, we used a unique approach, where the T-lymphokine stimulated altered responsiveness of keratinocytes was investigated [71]. The applied lymphokine mixture consisted INF- $\gamma$, an inflammatory mediator, which facilitates the proliferation of immature keratinocytes, and its effect was potentiated by IL-3 and GM-CSF. These data suggest that the listed lymphokines might implicated in the early disorder pathogenesis, thus, our microarray experimental design was suitable to describe novel factors participating in the primary phase of psoriasis [71, 72]. Currently, the IL-17/23/IL22 axis and TNF- $\alpha$ attracts more attention in psoriasis compared to INF- $\gamma$, but it is unquestionable that IFN- $\gamma$ plays an essential role in disorder initiation and establishement. However, to reveal its exact mechanism of action in psoriasis, further studies are needed $[6,19]$.

Among differentially regulated genes between the healthy and psoriatic non-involved epidermis, we identifed three SR-rich splicing regulators, LUC7L3, PPIG and SFRS18. This finding is of special interest, as neither the splicing disturbances nor the biological function of these SR-rich splicing regulators have been characterized in depth in psoriasis. In the Results section, we demonstrated that splicing regulators are downregulated or do not show any changes in psoriatic non-involved epidermis upon T-lymphokine treatment, as compared to healthy tissue specimens. We described the same phenomenon in case of several transcripts identified in our cDNA microarray experiment. This seeming contradiction could be explained by the following: the gene products are already upregulated in the non-involved epidermis, hence, the lymphokine treatment does not result in further elevation of expression [71]. Furthermore, an upregulation tendency of splicing regulators was revealed for splicing regulators in psoriatic non-involved epidermis.

Besides the participation in mRNA maturation, LUC7L3 influences other molecular processes, for instance, it is involved in cysplatine resistence of tumor cells [82, 83, 84]. Novel data suggests that it might behave as an antiviral compound via inhibition of hepatitis-B virus 
replication [85]. Moreover, LUC7L3 could be one of the protein interactors of cAMP responsive element (CRE), thus, it may play a role in cellular signal transduction processes [86].

PPIG is implicated in protein folding, and its cell-cycle dependent phosphorylation via SRPK1 and SRPK3 is also described [87, 88]. Literature and data mining indicates that PPIG together with SFRS18 (also known as PNISR) might be physical and functional interactors of PNN, a desmosome-associated particle, playing roles in splicing and cellular connections, repectively $[88,89]$.

The previous results of the research group already indicated that certain mRNA isoforms might promote psoriasis development. We and others have previously verified that the fibronectin splice variant containing the oncofetal EDA domain (EDA+ fibronectin) might be responsible for the altered responsiveness of the psoriatic non-involved epidermis. The amount of the EDA domain is elevated in non-involved epidermis compared to healthy skin, and we also demonstrated that keratinocytes are effective producers of this domain [37, 50]. Regulation of fibronectin splicing has been studied in detail, however, our knowledge regarding the formation of its isoform is still incomplete [40]. Other reports described, that TGF- $\beta$ and another type of SR-rich protein (SF2/ASF) promote the EDA domain inclusion while snRNPs possess an opposite effect $[39,90,91,92,93]$. STAT1 promotes EDA inclusion in fibroblast supported by the current data of our research group [94].

In the first study presented in the dissertation, we aimed to clarify whether SR-rich splicing regulators identified to be differentially regulated in psoriasis are implicated in the production of the EDA+ fibronectin. In addition, investigation of expression characteristics of $L U C 7 L 3$, $P P I G$ and SFRS18 was another aim of our study. We observed their gene expression differences among healthy, psoriatic non-involved and involved epidermis, and the expression of splicing regulators was also monitored during keratinocyte proliferation/differentiation phases of immortalized keratinocytes. By comparing splicing factor abundance in different types of epidermis samples, we concluded that all of the three splicing regulators are upregulated in the involved epidermis, exhibiting approximately 2-3 fold elevation. LUC7L3 showed a moderate, but significant increase in non-involved epidermis, which can suggest its association with EDA+ fibronectin. In contrast to LUC7L3, PPIG is diminished in the non-involved epidermis, therefore, its pattern of expression resembles that of STAT1, moreover, both STAT1 and PPIG 
are the component of the molecular network including keratinocyte growth factor (KGF) and fibronectin signalling [94].

The congruent expression of the three splicing factors in synchronized keratinocytes is a remarkable outcome of our study. As LUC7L3, PPIG and SFRS18 are localized on different chromosomes, genetic linkage is not liable for the experienced similarities, and leaves way to alternative explanation for the phenomenon. The most plausible answer is that these SR-rich splicing factors might share similar upstream regulatory elements, including transcription factors and their binding sites. If we consider the results of the Western blot and immunostaining experiments together, the expression of splicing regulators both in involved epidermis and immortalized keratinocytes is well-defined. This finding suggests that abundant LUC7L3, PPIG and SFRS18 expression might be involved in the shortened and disturbed maturation of keratinocytes that is typical of psoriatic skin and is also an attribute of the immortalized HPV-KER cells.

We constructed an in vitro experimental system to analyze whether altered expression of LUC7L3, PPIG and SFRS18 influenced the ratio of the oncofetal and normal fibronectin isoforms. siRNA silencing of these splicing factors verified, that single silencing leads to significant decrease in the relative amount of the EDA+ fibronectin isoform, which is present in majority in the immature keratinocytes. Moreover, we also investigated the effects of combined silencing, to observe the existence of synergistic effects. The previous analysis of publicly available databases supported the relationship of LUC7L3 and SFRS18, and accordingly to the prediction, double silencing of LUC7L3/SFRS18 enhanced the decrease of EDA domain inclusion. Triple silecing did not cause significant difference compared to the combined silencing of LUC7L3 and SFRS18. Using the LUC7L3/SFRS18 double-silenced combination, we tested whether the fibronectin related isoform alterations are also present at protein level. Flow cytometry validated the mRNA-level findings, since we were able to demonstrate the decrease of EDA+ fibronectin amount in HPV-KER keratinocytes, whithout changing the quantity of total fibronectin: this result refers to an altered mRNA maturation mechanism in the background. Based on prior reports, SR-rich proteins induces exon inclusion via interaction of enhancer splicing elements, therefore the finding possess special importance [95, 96, 97]. 
Findings introduced so far suggested, that the SR-rich proteins might be implicated in psoriasis in multiple manners, thus, tissue stainings and the results related to synchronized keratinocytes and mRNA maturation of fibronectin prompted us to examine other pathways influenced by the aforementioned splicing factors. To obtain a deeper insight into molecular functions associated with splicing regulators, we applied again a high-throughput gene expression tool, paired-end RNA-Sequencing. For further analysis, we have chosen for further analysis the double silencing of $L U C 7 L 3 / S F R S 18$, which resulted in the most robust splicing pattern alterations regarding the $\mathrm{EDA}+$ fibronectin formation.

In accordance with our primary hypothesis, siRNA mediated silencing of LUC7L3/SFRS18 caused relatively modest changes in gene expression, and definite alterations in splicing patterns. However, several significant results were obtained concerning differential expression, the most important one is related to an interferon-inducible gene, the IFI6 (G1P3). IFI6 belongs to the family of interferon-stimulated genes (ISGs), together with ISG12A, ISGI2B, ISGI2C. $I S G 12 A$ is also referred as IFI27 [98]. In contrast to other members of ISGs, IFI6 was shown to be rather antiapoptotic: this feature was observed in cancer cells, and our research group presented its overexpression both in non-involved and involved epidermis [99, 100]. Via elevated expression IFI6 could be responsible for the survival of immature keratinocytes which is an important phenomenon in psoriasis. Moreover, we have also analyzed the interaction of G1P3 and PRINS, a non-coding RNA implicated in the cellular stress responses of keratinocytes [99, 101]. Our RNA-Seq results enrich our knowledge on the relation of IFI6: in response to $L U C 7 L 3 / S F R S 18$, overexpression of IFI6 could be observed, moreover the result have been validated in independent samples as well. Real-Time RT-PCR indicated up to 4-fold upregulation in siRNA silenced samples as compared to the scrambled controls. An important coincidence is that we could also detect the upregulation of the apoptotic IFI27, which exerts its effect via Bax activation and causes mitochondrial cytocin-c release, and decrease the mitochondial membrane potential [98]. Function of IFI27 has been also assessed in psoriasis: some experimental data suggest high abundance in psoriatic skin samples [102]. Taken together, we can conclude that SR-rich proteins could be involved in the fine-tuning of pro- and antiapoptotic events in human keratinocytes. This is not the only proof for interferon-SR-rich splicing factor connection, since a previous report demonstrated that serine/arginine-rich 
splicing factor 1 (SRSF1) facilitated the type-I IFN production, via the double-stranded RNA sensor RIG-1, which is also an abundant molecular component in psoriasis [103].

We also analysed the predicted interactors of IFI6 using the STRING database (Fig. 19.). During the review of the gene list and its comparison to the RNA-Seq results, we found that several of them exerted significant upregulation upon silencing. OAS1, OAS2, IFIT1, IFIT3, ISG15, MX1 were all identified to be upregulated in our RNA-Seq experiment, besides that, literature data supported their implication in antiviral immunity [104, 105]. ISG15, MX1 and OAS2 was previously decribed to be upregulated in psoriatic epidermis as compared to the healthy samples [104]. To date, Raposo and his co-workers demonstrated in their recent RNASeq study that 16 antiviral genes exhibit at least two-fold elevation in psoriatic epidermis compared to the healtly and non-involved epidermis samples [105]. This gene list also includes ISG15. The results related to the antiviral immunity might have a huge importance, considering that novel experimental data suggests that LUC7L3 inhibits hepatitis B virus replication [85]. Furthermore, there is several evidence that psoriatic skin is less prone to viral infections due to the upregulation of the type I interferon induced antiviral pathway [104, 105].

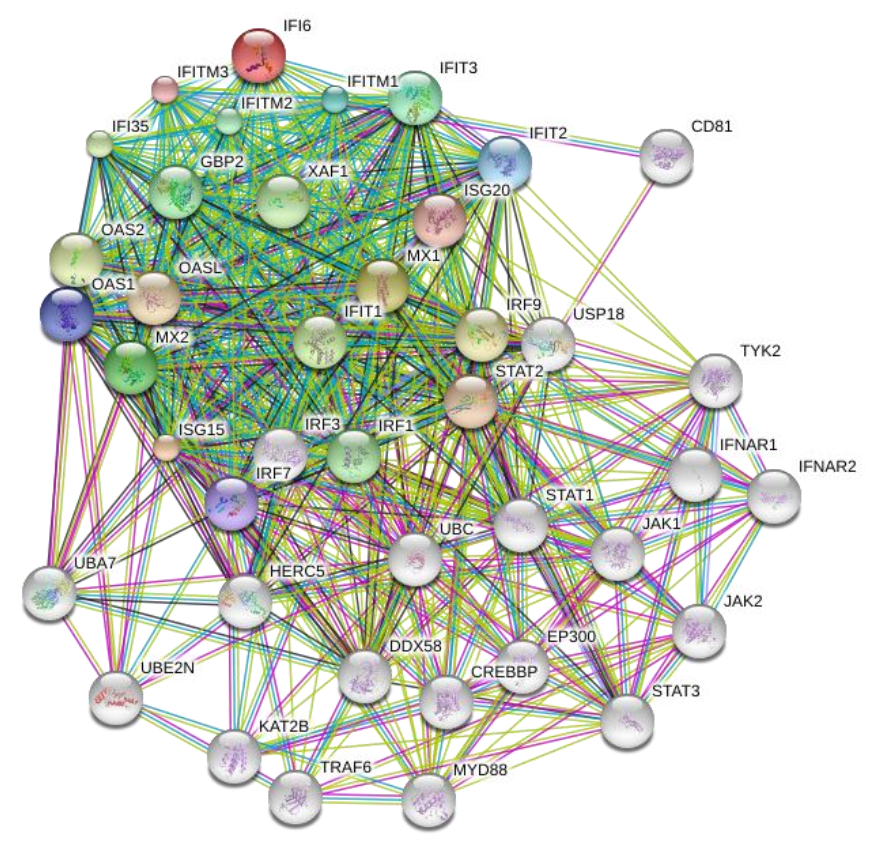

Fig. 19. Interaction network of IFI6 predicted by STRING database (https://stringdb.org/cgi/input.pl?taskId=_notask\&UserId=gz5TU6vnllRU\&sessionId=FpUklt2OTNm7) 
In our RNA-Seq experiments we also demonstrated, that innate immunity components, like KRT6A and the calcium binding protein S100A9 are regulated by LUC7L3/SFRS18. S100A9 forms a heterodimeric complex with S100A8, and together, they exert antimicrobial and chemoattractant functions [106, 107]. S100A9 and KRT6A belong to the same molecular network related to barrier function, innate immunity and danger signals. KRT6A is also involved in wound healing processes [108].

Splicing pattern alteration related to the EDA+ fibronectin isoform (the drop of inclusion tendency) was reproducible in the samples of RNA-Seq experiment. Besides that, we were also able to demonstrate the elevated level for integrin 5 (ITGA5), the known fibronectin receptor, also having the capacity to bind the EDA domain of fibronectin. As consequence of the diminished relative amount of the EDA+ fibronectin, ITGA5 abundance is elevated, which could be considered to be a cellular compensatory mechanism. This phenomenon is a novel evidence for the suggestion, that EDA domain inclusion alters the binding partners of fibronectin [39, 41, 42, 45]. Previous data indicated that the presence of the EDA domain increases the affinity towards $\alpha 5 \beta 1$ integrin receptor, which is the most relevant fibronectin interactor $[42,43,45]$. However, a contradicting report claims that the EDA+ fibronectin does not bind to this receptor, and the $\alpha 4 \beta 1$ and $\alpha 9 \beta 1$ integrins recognize the EDGIHEL sequence located in the EDA domain. Additional results support the attachment of EDA+ fibronectin to the innate immune system component TLR4 receptor $[44,45,46]$. The complexity of this regulatory network is indicated by the fact that the effect of S100A9 - upregulated also in response to $L U C 7 L 3 / S F R 18$ silencing - is also mediated by TLR4 [106, 107]. Moreover, other fibronectin signalling related components, as syndecan-4 (SDC4) and tenascin-C show altered expression upon silencing of splicing factors [109].

LUC7L3, which was formerly designated as CREAP-1, contains a binding domain for the cAMP regulatory element (CRE) [86]. However, based on silencing experiments, it may also have influence on the mRNA maturation of CREB1, besides the potential direct interplay with CRE. Therefore LUC7L3 potentially exerts a modulatory role on CREB1: altered splicing might regulate the protein binding of this fundamental element of transcription. In addition, various cellular processes are affected by differential exon usage, such as CUL1, HERC1 and HERC6 implicated in ubiquitination. CUL1 is part of the E3 ubiquitin ligase complex and known to be overexpressed in melanoma, mediating hyperproliferative signals [110]. The 
HERC gene family contributes to multiple molecular pathways, including DNA repair, cellcycle regulation, and ubiquitination. Köks and co-workers demonstrated the isoform ratio changes of HERC6 in psoriatic skin compared to healthy samples [69]. HERC6 is also referred in E3 conjugation of ISG15 [111]. The antiviral OAS2 exhibited not only upregulation but differential exon usage as well. Multiple genes with relatively sparse functional annotation available in the present literature exerted markedly altered exon usage. These comprises the HERC2 paralog RCC1, NBEAL1 and AUH, with RNA-binding and hydratase activities [112, $113,114]$.

Using RNA-Seq, exon usage alterations of long non-coding RNAs (lncRNAs) are also demonstrable [70, 115]. In our study, we have demonstrated several non-coding RNAs with differential exon usage, such as NEAT1 and TINCR. The contribution of NEAT1 to tumorigenesis is well studied: its overexpression has been validated in esophagus, colorectal, hepatocellularand lung carcinoma [116]. Since hyperproliferation of keratinocytes is a rudimental step in the pathogenesis, NEAT1 could presumably act in psoriasis pathogenesis.

The widespread functionality of TINCR in keratinocyte maturation has been currently delieneated: in contrast with another non-coding transcript, ANCR, which is required for the maintenance of keratinocyte precursors, TINCR promotes terminal differentiation. TINCR is a low-abundance transcript in undifferentiated keratinocytes, and shows increase in mature keratinocytes [117, 118]. Moreover, TINCR and its modulated gene STAU1 exerted elevated expression in the psoriatic gene module, based on a WGCNA gene network analysis of a former RNA-Seq [68]. We have demonstrated that TINCR is present at low levels in HPV immortalized keratinocytes. The binding capacity of TINCR is determined by several „TINCR box" motifs, and inclusion or exclusion of these motifs by altered splicing might modulate binding features [118]. 


\section{CONCLUSION}

In recent years, we gained a deeper insight into psoriasis pathogenesis. Much of our current knowledge based on wide-spread application of high-throughput methods, including cDNA microarrays and RNA-Seq, however, the initiation phase of the disorder is still only partially uncovered. In our recent microarray study, three splicing regulators - luc-7 like protein 3 (LUC7L3), peptidyl-prolyl cis-trans isomerase G (PPIG) and arginine/serine-rich 18 (SFRS18) - have been identified, which may be accountable for the pathogenic response of keratinocytes to T-lymphokine stimuli. Subsequent characterization of expression revealed the definite presence of splicing regulators in immature keratinocytes of cell lines and psoriatic epidermis, suggesting their multiple involvement in disorder pathogenesis. Furthermore, LUC7L3, PPIG and SFRS18 might share common upstream regulatory elements.

It was also proved that SR-rich splicing factors function as regulators of the fibronectin mRNA maturation, and facilitate inclusion of the disease-associated EDA domain. In this process, a synergistic interaction has been confirmed for LUC7L3 and SFRS18. Thus, the subsequent RNA-Seq experiment on LUC7L3/SFRS18 double silenced cells showed the influence of splicing factors on several well-known psoriasis-associated pathways, especially IFN signalling, antiviral immunity, and ubiquitination. These could be responsible for the balancing between pro- and antiapoptotic events in keratinocytes, determine keratinocyte differentiation as in case of lncRNA TINCR, and could be part from multiple feed-back loops, responsible for the maintenance of molecular discrepancies seen in the disease. These findings are of special interest considering that interferon-related molecular abnormalities are not clarified yet in detail, however, their role in disease pathogenesis besides TNF- $\alpha$ and the IL17/IL23/IL22 axis is undoubted. LUC7L3, PPIG and SFRS18 might be contributors of disorder initiation, but presumably also participate in the establishment phases. 


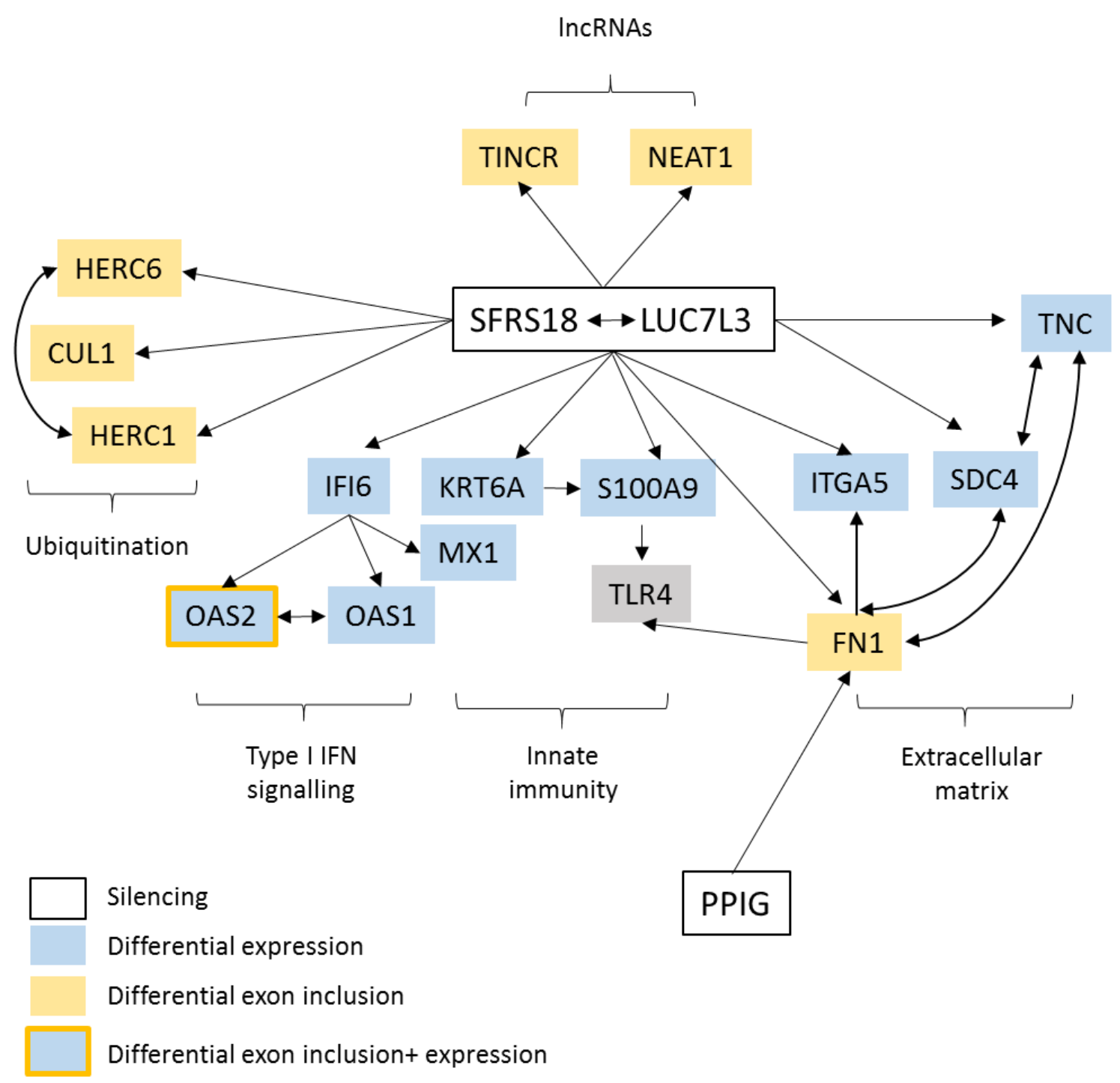

Fig. 20. Schematic model of molecular pathways, which might be influenced by LUC7L3, PPIG, and SFRS18 splicing regulators during psoriasis development 


\section{ACKNOWLEDGEMENT}

Foremost I thank my supervisor, Prof. Dr. Márta Széll for her continuous motivation and guidance throughout the Ph.D. program, without her support any of the presented work would have been impossible.

I am grateful for the support of Prof. Dr. Lajos Kemény and Prof. Dr. Zsuzsanna Bata-Csörgö for providing the background for the current study and for the practical help of Dr. Kornélia Szabó and Dr. Gergely Groma during the experimental work. I would like thank Dr. Franco Pagani for the possibility to spend a studentship in ICGEB Trieste to practice research techniques facilitating the preparation of the Ph.D. thesis. I am also grateful to Prof. Dr. Rolland Gyulai for the encouragement of Ph.D. work during my resident years at University of Pécs.

I would like to thank all my colleagues and staff of the Department of Dermatology and Allergology for their kind support.

I would like to also express gratitude to Prof. Dr. Anna Borsodi for the help in the start of my scientific work and the continous kind support.

Last, I give my special thanks to my loving husband, Péter, who participated in the bioinformatics workflow in the present study and my supporting family, as well.

Illumina short-read library preparation, sequencing and primary bioinformatic analysis of the samples was performed at the Genomic Medicine and Bioinformatic Core Facility of the University of Debrecen, Faculty of Medicine.

The study was supported by OTKA K105985, OTKA K111885 and TÁMOP-4.2.2.A11/1/KONV-2012-0035, GINOP-2.3.2-15-2016-00015 research grants. 


\section{REFERENCES}

1. Nestle FO, Kaplan DH, Barker J. Psoriasis. N Engl J Med. 2009 Jul 30;361(5):496-509.

2. Girolomoni G, Griffiths CE, Krueger J, Nestle FO, Nicolas JF, Prinz JC, Puig L, Ståhle M, van de Kerkhof PC, Allez M, Emery P, Paul C. Early intervention in psoriasis and immunemediated inflammatory diseases: A hypothesis paper. J Dermatolog Treat. 2015 Apr;26(2):10312.

3. Griffiths CE, Christophers E, Barker JN, Chalmers RJ, Chimenti S, Krueger GG, Leonardi C, Menter A, Ortonne JP, Fry L. A classification of psoriasis vulgaris according to phenotype. Br J Dermatol. 2007 Feb;156(2):258-62.

4. Di Meglio P, Villanova F, Nestle FO. Psoriasis. Cold Spring Harb Perspect Med. 2014 Aug $1 ; 4(8)$.

5. Greb JE, Goldminz AM, Elder JT, Lebwohl MG, Gladman DD, Wu JJ, Mehta NN, Finlay AY, Gottlieb AB. Psoriasis. Nat Rev Dis Primers. 2016 Nov 24;2:16082.

6. Lowes MA, Russell CB, Martin DA, Towne JE, Krueger JG. The IL-23/T17 pathogenic axis in psoriasis is amplified by keratinocyte responses. Trends Immunol. 2013 Apr;34(4):174-81.

7. Furue M, Kadono T. "Inflammatory skin march" in atopic dermatitis and psoriasis. Inflamm Res. 2017 Jun 15.

8. Esposito M, Saraceno R, Giunta A, Maccarone M, Chimenti S. An Italian study on psoriasis and depression. Dermatology. 2006;212(2):123-7.

9. Hunter HJ, Griffiths CE, Kleyn CE. Br J Dermatol. Does psychosocial stress play a role in the exacerbation of psoriasis? 2013 Nov;169(5):965-74.

10. Roberson ED, Bowcock AM. Psoriasis genetics: breaking the barrier. Trends Genet. 2010 Sep;26(9):415-23

11. Ray-Jones H, Eyre S, Barton A, Warren RB. One SNP at a Time: Moving beyond GWAS in Psoriasis. J Invest Dermatol. 2016 Mar;136(3):567-73. 
12. Weiss G, Shemer A, Trau H. The Koebner phenomenon: review of the literature. J Eur Acad Dermatol Venereol. 2002 May;16(3):241-8

13. Gudjonsson JE, Thorarinsson AM, Sigurgeirsson B, Kristinsson KG, Valdimarsson H. Streptococcal throat infections and exacerbation of chronic plaque psoriasis: a prospective study. Br J Dermatol. 2003 Sep;149(3):530-4.

14. Dellavalle RP, Johnson KR. Do smoking, obesity, and stress cause psoriasis? J Invest Dermatol. 2005 Jul;125(1):vi-vii.

15. Kim GK, Del Rosso JQ. Drug-provoked psoriasis: is it drug induced or drug aggravated?: understanding pathophysiology and clinical relevance. J Clin Aesthet Dermatol. 2010 Jan;3(1):32-8.

16. Schlaak JF, Buslau M, Jochum W, Hermann E, Girndt M, Gallati H, Meyer zum Büschenfelde KH, Fleischer B.T cells involved in psoriasis vulgaris belong to the Th1 subset. J Invest Dermatol. 1994 Feb;102(2):145-9.

17. Austin LM, Ozawa M, Kikuchi T, Walters IB, Krueger JG. The majority of epidermal T cells in Psoriasis vulgaris lesions can produce type 1 cytokines, interferon-gamma, interleukin2, and tumor necrosis factor-alpha, defining TC1 (cytotoxic T lymphocyte) and TH1 effector populations: a type 1 differentiation bias is also measured in circulating blood $\mathrm{T}$ cells in psoriatic patients. J Invest Dermatol. 1999 Nov;113(5):752-9.

18. Di Cesare A, Di Meglio P, Nestle FO.The IL-23/Th17 axis in the immunopathogenesis of psoriasis. J Invest Dermatol. 2009 Jun;129(6):1339-50.

19. Lowes MA, Suárez-Fariñas M, Krueger JG. Immunology of psoriasis. Annu Rev Immunol. 2014;32:227-55.

20. Zaba LC, Cardinale I, Gilleaudeau P, Sullivan-Whalen M, Suárez-Fariñas M, FuentesDuculan J, Novitskaya I, Khatcherian A, Bluth MJ, Lowes MA, Krueger JG. Amelioration of epidermal hyperplasia by TNF inhibition is associated with reduced Th17 responses. J Exp Med. 2007 Dec 24;204(13):3183-94

21. Haider AS, Cohen J, Fei J, Zaba LC, Cardinale I, Toyoko K, Ott J, Krueger JG. Insights into gene modulation by therapeutic TNF and IFNgamma antibodies: TNF regulates 
IFNgamma production by T cells and TNF-regulated genes linked to psoriasis transcriptome. $\mathbf{J}$ Invest Dermatol. 2008 Mar;128(3):655-66

22. Griffiths CE, Strober BE, van de Kerkhof P, Ho V, Fidelus-Gort R, Yeilding N, Guzzo C, Xia Y, Zhou B, Li S, Dooley LT, Goldstein NH, Menter A; ACCEPT Study Group. Comparison of ustekinumab and etanercept for moderate-to-severe psoriasis. N Engl J Med. 2010 Jan $14 ; 362(2): 118-28$

23. Nograles KE, Krueger JG. Anti-cytokine therapies for psoriasis. Exp Cell Res. 2011 May 15;317(9):1293-300.

24. Lande R, Gregorio J, Facchinetti V, Chatterjee B, Wang YH, Homey B, Cao W, Wang YH, Su B, Nestle FO, Zal T, Mellman I, Schröder JM, Liu YJ, Gilliet M.Plasmacytoid dendritic cells sense self-DNA coupled with antimicrobial peptide. Nature. 2007 Oct 4;449(7162):5649 .

25. Ganguly D, Chamilos G, Lande R, Gregorio J, Meller S, Facchinetti V, Homey B, Barrat FJ, Zal T, Gilliet M. Self-RNA-antimicrobial peptide complexes activate human dendritic cells through TLR7 and TLR8. J Exp Med. 2009 Aug 31;206(9):1983-94.

26. Farkas A, Kemény L. Interferon- $\alpha$ in the generation of monocyte-derived dendritic cells: recent advances and implications for dermatology. Br J Dermatol. 2011 Aug;165(2):247-54.

27. Wolf R, Howard OM, Dong HF, Voscopoulos C, Boeshans K, Winston J, Divi R, Gunsior M, Goldsmith P, Ahvazi B, Chavakis T, Oppenheim JJ, Yuspa SH. Chemotactic activity of S100A7 (Psoriasin) is mediated by the receptor for advanced glycation end products and potentiates inflammation with highly homologous but functionally distinct S100A15. J Immunol. 2008 Jul 15;181(2):1499-506.

28. Pruenster M, Vogl T, Roth J, Sperandio M. S100A8/A9: From basic science to clinical application. Pharmacol Ther. 2016 Nov;167:120-131.

29. Nestle FO, Conrad C, Tun-Kyi A, Homey B, Gombert M, Boyman O, Burg G, Liu YJ, Gilliet M. Plasmacytoid predendritic cells initiate psoriasis through interferon-alpha production. J Exp Med. 2005 Jul 4;202(1):135-43. 
30. Wilson NJ, Boniface K, Chan JR, McKenzie BS, Blumenschein WM, Mattson JD, Basham B, Smith K, Chen T, Morel F, Lecron JC, Kastelein RA, Cua DJ, McClanahan TK, Bowman EP, de Waal Malefyt R. Development, cytokine profile and function of human interleukin 17producing helper T cells. Nat Immunol. 2007 Sep;8(9):950-7.

31. Zheng Y, Danilenko DM, Valdez P, Kasman I, Eastham-Anderson J, Wu J, Ouyang W. Interleukin-22, a $\mathrm{T}(\mathrm{H}) 17$ cytokine, mediates IL-23-induced dermal inflammation and acanthosis. Nature. 2007 Feb 8;445(7128):648-51.

32. Boyman O, Conrad C, Tonel G, Gilliet M, Nestle FO. The pathogenic role of tissue-resident immune cells in psoriasis. Trends Immunol. 2007 Feb;28(2):51-7.

33. Man XY, Yang XH, Cai SQ, Bu ZY, Zheng M. Overexpression of vascular endothelial growth factor (VEGF) receptors on keratinocytes in psoriasis: regulated by calcium independent of VEGF. J Cell Mol Med. 2008 Apr;12(2):649-60

34. Johnston A, Fritz Y, Dawes SM, Diaconu D, Al-Attar PM, Guzman AM, Chen CS, Fu W, Gudjonsson JE, McCormick TS, Ward NL. Keratinocyte overexpression of IL-17C promotes psoriasiform skin inflammation. J Immunol. 2013 Mar 1;190(5):2252-62.

35. Halprin KM. Epidermal "turnover time"--a re-examination. Br J Dermatol. 1972 Jan;86(1):14-9.

36. Latijnhouwers MA, de Jongh GJ, Bergers M, de Rooij MJ, Schalkwijk J. Expression of tenascin-C splice variants by human skin cells. Arch Dermatol Res. 2000 Sep;292(9):446-54.

37. Ting KM, Rothaupt D, McCormick TS, Hammerberg C, Chen G, Gilliam AC, Stevens S, Culp L, Cooper KD. Overexpression of the oncofetal Fn variant containing the EDA splice-in segment in the dermal-epidermal junction of psoriatic uninvolved skin. J Invest Dermatol. 2000 Apr;114(4):706-11.

38. Kornblihtt AR, Pesce CG, Alonso CR, Cramer P, Srebrow A, Werbajh S, Muro AF. The fibronectin gene as a model for splicing and transcription studies. FASEB J. 1996 Feb;10(2):248-57.

39. White ES, Baralle FE, Muro AF. New insights into form and function of fibronectin splice variants. J Pathol. 2008 Sep;216(1):1-14. 
40. White ES, Muro AF. Fibronectin splice variants: understanding their multiple roles in health and disease using engineered mouse models. IUBMB Life. 2011 Jul;63(7):538-46.

41. Xu J, Mosher D (2011) The Extracellular Matrix: an Overview. Biology of extracellular matrix. Springer-Verlag Berlin, Heidelberg, pp 41-76

42. Pankov R, Yamada KM. Fibronectin at a glance. J Cell Sci. 2002 Oct 15;115(Pt 20):38613

43. Pellegrini G, De Luca M, Orecchia G, Balzac F, Cremona O, Savoia P, Cancedda R, Marchisio PC. Expression, topography, and function of integrin receptors are severely altered in keratinocytes from involved and uninvolved psoriatic skin. J Clin Invest. 1992 Jun;89(6):1783-95.

44. Okamura Y, Watari M, Jerud ES, Young DW, Ishizaka ST, Rose J, Chow JC, Strauss JF 3rd.The extra domain A of fibronectin activates Toll-like receptor 4. J Biol Chem. 2001 Mar 30;276(13):10229-33.

45. McFadden JP, Basketter DA, Dearman RJ, Kimber IR. Extra domain A-positive fibronectin-positive feedback loops and their association with cutaneous inflammatory disease. Clin Dermatol. 2011 May-Jun;29(3):257-65.

46. Kelsh RM, McKeown-Longo PJ, Clark RA. EDA Fibronectin in Keloids Create a Vicious Cycle of Fibrotic Tumor Formation. J. Invest Dermatol. 2015 Jul;135(7):1714-8.

47. Muro AF, Chauhan AK, Gajovic S, Iaconcig A, Porro F, Stanta G, Baralle FE. Regulated splicing of the fibronectin EDA exon is essential for proper skin wound healing and normal lifespan. J Cell Biol. 2003 Jul 7;162(1):149-60.

48. Ffrench-Constant C, Van de Water L, Dvorak HF, Hynes RO. Reappearance of an embryonic pattern of fibronectin splicing during wound healing in the adult rat. J Cell Biol. 1989 Aug;109(2):903-14.

49. Astrof S, Crowley D, George EL, Fukuda T, Sekiguchi K, Hanahan D, Hynes RO. Direct test of potential roles of EIIIA and EIIIB alternatively spliced segments of fibronectin in physiological and tumor angiogenesis. Mol Cell Biol. 2004 Oct;24(19):8662-70. 
50. Széll M, Bata-Csörgo Z, Koreck A, Pivarcsi A, Polyánka H, Szeg C, Gaál M, Dobozy A, Kemény L. Proliferating keratinocytes are putative sources of the psoriasis susceptibilityrelated EDA+ (extra domain A of fibronectin) oncofetal fibronectin. J Invest Dermatol. 2004 Sep;123(3):537-46.

51. Kunz M, Ibrahim SM, Koczan D, Scheid S, Thiesen HJ, Gross G. DNA microarray technology and its applications in dermatology. Exp Dermatol. 2004 Oct;13(10):593-606.

52. Blumenberg M. SKINOMICS: Transcriptional Profiling in Dermatology and Skin Biology. Curr Genomics. 2012 Aug;13(5):363-8

53. Villaseñor-Park J, Ortega-Loayza AG. Microarray technique, analysis, and applications in dermatology. J Invest Dermatol. 2013 Apr;133(4):e7.

54. Quigley D. RNA-seq permits a closer look at normal skin and psoriasis gene networks. J Invest Dermatol. 2014 Jul;134(7):1789-91

55. Nomura I, Gao B, Boguniewicz M, Darst MA, Travers JB, Leung DY. Distinct patterns of gene expression in the skin lesions of atopic dermatitis and psoriasis: a gene microarray analysis. J Allergy Clin Immunol. 2003 Dec;112(6):1195-202.

56. Zhou X, Krueger JG, Kao MC, Lee E, Du F, Menter A, Wong WH, Bowcock AM. Novel mechanisms of T-cell and dendritic cell activation revealed by profiling of psoriasis on the 63,100-element oligonucleotide array. Physiol Genomics. 2003 Mar 18;13(1):69-78.

57. Baechler EC, Batliwalla FM, Reed AM, Peterson EJ, Gaffney PM, Moser KL, Gregersen PK, Behrens TW. Gene expression profiling in human autoimmunity. Immunol Rev. 2006 Apr;210:120-37.

58. Gudjonsson JE, Ding J, Li X, Nair RP, Tejasvi T, Qin ZS, Ghosh D, Aphale A, Gumucio DL, Voorhees JJ, Abecasis GR, Elder JT. Global gene expression analysis reveals evidence for decreased lipid biosynthesis and increased innate immunity in uninvolved psoriatic skin. $\mathbf{J}$ Invest Dermatol. 2009 Dec;129(12):2795-804.

59. Zaba LC, Fuentes-Duculan J, Eungdamrong NJ, Abello MV, Novitskaya I, Pierson KC, Gonzalez J, Krueger JG, Lowes MA. Psoriasis is characterized by accumulation of 
immunostimulatory and Th1/Th17 cell-polarizing myeloid dendritic cells. J Invest Dermatol. 2009 Jan;129(1):79-88.

60. Zaba LC, Fuentes-Duculan J, Eungdamrong NJ, Johnson-Huang LM, Nograles KE, White TR, Pierson KC, Lentini T, Suárez-Fariñas M, Lowes MA, Krueger JG. Identification of TNFrelated apoptosis-inducing ligand and other molecules that distinguish inflammatory from resident dendritic cells in patients with psoriasis. J Allergy Clin Immunol. 2010 Jun;125(6):1261-1268.e9

61. Reisch1 J, Schwenke S, Beekman JM, Mrowietz U, Stürzebecher S, Heubach JF. Increased expression of Wnt5a in psoriatic plaques. J Invest Dermatol. 2007 Jan;127(1):163-9.

62. Buerger C, Malisiewicz B, Eiser A, Hardt K, Boehncke WH. Mammalian target of rapamycin and its downstream signalling components are activated in psoriatic skin. $\mathrm{Br} \mathbf{J}$ Dermatol. 2013 Jul;169(1):156-9.

63. Goldminz AM, Au SC, Kim N, Gottlieb AB, Lizzul PF. NF-кB: an essential transcription factor in psoriasis. J Dermatol Sci. 2013 Feb;69(2):89-94.

64. Romanowska M, al Yacoub N, Seidel H, Donandt S, Gerken H, Phillip S, Haritonova N, Artuc M, Schweiger S, Sterry W, Foerster J. PPARdelta enhances keratinocyte proliferation in psoriasis and induces heparin-binding EGF-like growth factor. J Invest Dermatol. 2008 Jan;128(1):110-24.

65. Tian S, Krueger JG, Li K, Jabbari A, Brodmerkel C, Lowes MA, Suárez-Fariñas M. Metaanalysis derived (MAD) transcriptome of psoriasis defines the "core" pathogenesis of disease. PLoS One. 2012;7(9):e44274

66. Manczinger M, Kemény L. Novel factors in the pathogenesis of psoriasis and potential drug candidates are found with systems biology approach. PLoS One. 2013 Nov 26;8(11):e80751

67. Jabbari A, Suárez-Fariñas M, Dewell S, Krueger JG. Transcriptional profiling of psoriasis using RNA-seq reveals previously unidentified differentially expressed genes. J Invest Dermatol. 2012 Jan;132(1):246-9

68. Li B, Tsoi LC, Swindell WR, Gudjonsson JE, Tejasvi T, Johnston A, Ding J, Stuart PE, Xing X, Kochkodan JJ, Voorhees JJ, Kang HM, Nair RP, Abecasis GR, Elder JT. 
Transcriptome analysis of psoriasis in a large case-control sample: RNA-seq provides insights into disease mechanisms. J Invest Dermatol. 2014 Jul;134(7):1828-38.

69. Kõks S, Keermann M, Reimann E, Prans E, Abram K, Silm H, Kõks G, Kingo K. PsoriasisSpecific RNA Isoforms Identified by RNA-Seq Analysis of 173,446 Transcripts. Front Med (Lausanne). 2016 Oct 7;3:46. eCollection 2016.

70. Antonini D, Mollo MR, Missero C. Research Techniques Made Simple: Identification and Characterization of Long Noncoding RNA in Dermatological Research. J Invest Dermatol. 2017 Mar;137(3):e21-e26.

71. Szabó K, Bata-Csörgő Z, Dallos A, Bebes A, Francziszti L, Dobozy A, Kemény L, Széll M. Regulatory networks contributing to psoriasis susceptibility. Acta Derm Venereol. 2014 Jul;94(4):380-5.

72. Bata-Csorgo Z, Hammerberg C, Voorhees JJ, Cooper KD. Kinetics and regulation of human keratinocyte stem cell growth in short-term primary ex vivo culture. Cooperative growth factors from psoriatic lesional $\mathrm{T}$ lymphocytes stimulate proliferation among psoriatic uninvolved, but not normal, stem keratinocytes. J Clin Invest. 1995 Jan;95(1):317-27.

73. Polyanka H, Szabo K, Tax G, Tubak V, Kusz E, Ujfaludi Z, Boros I, Bata-Csorgo Z, Kemeny L, Szell M. Primary characterization of a novel HPV- E6 oncogene immortalized keratinocyte cell line. J Invest Dermatol 2011 131: (Suppl. 2) p. S70

74. Pivarcsi A, Széll M, Kemény L, Dobozy A, Bata-Csörgo Z. Serum factors regulate the expression of the proliferation-related genes alpha5 integrin and keratin 1, but not keratin 10 , in HaCaT keratinocytes. Arch Dermatol Res. 2001 Apr;293(4):206-13.

75. Alexander Dobin, Carrie A. Davis, Felix Schlesinger, Jorg Drenkow, Chris Zaleski, Sonali Jha, Philippe Batut, Mark Chaisson, Thomas R. Gingeras. STAR: ultrafast universal RNA-seq aligner. Bioinformatics. 2013 Jan; 29(1): 15-21.

76. Daehwan Kim, Geo Pertea, Cole Trapnell, Harold Pimentel, Ryan Kelley, Steven L Salzberg. TopHat2: accurate alignment of transcriptomes in the presence of insertions, deletions and gene fusions. Genome Biol. 2013; 14(4): R36. 
77. Trapnell C, Williams BA, Pertea G, Mortazavi A, Kwan G, van Baren MJ, Salzberg SL, Wold BJ, Pachter L. Transcript assembly and quantification by RNA-Seq reveals unannotated transcripts and isoform switching during cell differentiation. Nat Biotechnol. 2010 May;28(5):511-5.

78. Anders S, Pyl PT, Huber W. HTSeq--a Python framework to work with high-throughput sequencing data. Bioinformatics. 2015 Jan 15;31(2):166-9.

79. Anders S, Reyes A, Huber W. Detecting differential usage of exons from RNA-seq data. Genome Res. 2012 Oct;22(10):2008-17.

80. Hooper JE. A survey of software for genome-wide discovery of differential splicing in RNA-Seq data. Hum Genomics. 2014 Jan 21;8:3.

81. Liu F, Gao X, Wang J, Gao C, Li X, Li X, Gong X, Zeng X. Transcriptome Sequencing to Identify Transcription Factor Regulatory Network and Alternative Splicing in Endothelial Cells Under VEGF Stimulation.J Mol Neurosci. 2016 Feb;58(2):170-7.

82. Nishii Y, Morishima M, Kakehi Y, Umehara K, Kioka N, Terano Y, Amachi T, Ueda K. CROP/Luc7A, a novel serine/arginine-rich nuclear protein, isolated from cisplatin-resistant cell line. FEBS Lett. 2000 Jan 14;465(2-3):153-6.

83. Umehara H, Nishii Y, Morishima M, Kakehi Y, Kioka N, Amachi T, Koizumi J, Hagiwara M, Ueda K. Effect of cisplatin treatment on speckled distribution of a serine/arginine-rich nuclear protein CROP/Luc7A. Biochem Biophys Res Commun. 2003 Feb 7;301(2):324-9.

84. Puig O, Bragado-Nilsson E, Koski T, Séraphin B. The U1 snRNP-associated factor Luc7p affects 5' splice site selection in yeast and human. Nucleic Acids Res. 2007;35(17):5874-85.

85. Li Y, Ito M, Sun S, Chida T, Nakashima K, Suzuki T. LUC7L3/CROP inhibits replication of hepatitis B virus via suppressing enhancer II/basal core promoter activity. Sci Rep. 2016 Nov $18 ; 6: 36741$.

86. Shipman KL, Robinson PJ, King BR, Smith R, Nicholson RC. Identification of a family of DNA-binding proteins with homology to RNA splicing factors. Biochem Cell Biol. 2006 Feb;84(1):9-19. 
87. Dubourg B, Kamphausen T, Weiwad M, Jahreis G, Feunteun J, Fischer G, Modjtahedi N. The human nuclear SRcyp is a cell cycle-regulated cyclophilin. J Biol Chem. 2004 May 21;279(21):22322-30.

88. Lin CL, Leu S, Lu MC, Ouyang P. Over-expression of SR-cyclophilin, an interaction partner of nuclear pinin, releases SR family splicing factors from nuclear speckles. Biochem Biophys Res Commun. 2004 Aug 27;321(3):638-47.

89. Zimowska G, Shi J, Munguba G, Jackson MR, Alpatov R, Simmons MN, Shi Y, Sugrue SP. Pinin/DRS/memA interacts with SRp75, SRm300 and SRrp130 in corneal epithelial cells. Invest Ophthalmol Vis Sci. 2003 Nov;44(11):4715-23.

90. Balza E, Borsi L, Allemanni G, Zardi L. Transforming growth factor beta regulates the levels of different fibronectin isoforms in normal human cultured fibroblasts. FEBS Lett. 1988 Feb 8;228(1):42-4

91. Borsi L, Castellani P, Risso AM, Leprini A, Zardi L. Transforming growth factor-beta regulates the splicing pattern of fibronectin messenger RNA precursor. FEBS Lett. 1990 Feb $12 ; 261(1): 175-8$.

92. Caputi M, Casari G, Guenzi S, Tagliabue R, Sidoli A, Melo CA, Baralle FE. A novel bipartite splicing enhancer modulates the differential processing of the human fibronectin EDA exon. Nucleic Acids Res. 1994 Mar 25;22(6):1018-22.

93. Buratti E, Muro AF, Giombi M, Gherbassi D, Iaconcig A, Baralle FE. RNA folding affects the recruitment of SR proteins by mouse and human polypurinic enhancer elements in the fibronectin EDA exon. Mol Cell Biol. 2004 Feb;24(3):1387-400.

94. Gubán B, Vas K, Balog Z, Manczinger M, Bebes A, Groma G, Széll M, Kemény L, BataCsörgö Z. Abnormal regulation of fibronectin production by fibroblasts in psoriasis. $\mathrm{Br} \mathrm{J}$ Dermatol. 2016 Mar;174(3):533-41.

95. Faustino NA, Cooper TA. Pre-mRNA splicing and human disease. Genes Dev. 2003 Feb $15 ; 17(4): 419-37$.

96. Pagani F, Baralle FE (2010) Analysis of Human Splicing Defects Using Hybrid Minigenes. Molecular Diagnostics. Elsevier, pp 155-169. 
97. Ward AJ, Cooper TA. The pathobiology of splicing. J Pathol. 2010 Jan;220(2):152-63.

98. Gytz H, Hansen MF, Skovbjerg S, Kristensen AC, Hørlyck S, Jensen MB, Fredborg M, Markert LD, McMillan NA, Christensen EI, Martensen PM. Apoptotic properties of the type 1 interferon induced family of human mitochondrial membrane ISG12 proteins. Biol Cell. 2017 Feb;109(2):94-112.

99. Szegedi K, Sonkoly E, Nagy N, Németh IB, Bata-Csörgo Z, Kemény L, Dobozy A, Széll M. The anti-apoptotic protein G1P3 is overexpressed in psoriasis and regulated by the noncoding RNA, PRINS. Exp Dermatol. 2010 Mar;19(3):269-78

100. Cheriyath V, Leaman DW, Borden EC. Emerging roles of FAM14 family members (G1P3/ISG 6-16 and ISG12/IFI27) in innate immunity and cancer. J Interferon Cytokine Res. 2011 Jan;31(1):173-81

101. Sonkoly E, Bata-Csorgo Z, Pivarcsi A, Polyanka H, Kenderessy-Szabo A, Molnar G, Szentpali K, Bari L, Megyeri K, Mandi Y, Dobozy A, Kemeny L, Szell M. Identification and characterization of a novel, psoriasis susceptibility-related noncoding RNA gene, PRINS. J Biol Chem. 2005 Jun 24;280(25):24159-67.

102. Bowcock AM, Shannon W, Du F, Duncan J, Cao K, Aftergut K, Catier J, Fernandez-Vina MA, Menter A. Insights into psoriasis and other inflammatory diseases from large-scale gene expression studies. Hum Mol Genet. 2001 Aug 15;10(17):1793-805.

103. Xue F, Li X, Zhao X, Wang L, Liu M, Shi R, Zheng J. SRSF1 facilitates cytosolic DNAinduced production of type I interferons recognized by RIG-I. PLoS One. 2015 Feb 6;10(2):e0115354.

104. Wolk K, Witte K, Witte E, Raftery M, Kokolakis G, Philipp S, Schönrich G, Warszawska K, Kirsch S, Prösch S, Sterry W, Volk HD, Sabat R. IL-29 is produced by T(H)17 cells and mediates the cutaneous antiviral competence in psoriasis. Sci Transl Med. 2013 Sep 25;5(204):204ra129

105. Raposo RA, Gupta R, Abdel-Mohsen M, Dimon M, Debbaneh M, Jiang W, York VA, Leadabrand KS, Brown G, Malakouti M, Arron S, Kuebler PJ, Wu JJ, Pillai SK, Nixon DF, 
Liao W. Antiviral gene expression in psoriasis. J Eur Acad Dermatol Venereol. 2015 Oct;29(10):1951-7.

106. Schonthaler HB, Guinea-Viniegra J, Wculek SK, Ruppen I, Ximénez-Embún P, GuíoCarrión A, Navarro R, Hogg N, Ashman K, Wagner EF. S100A8-S100A9 protein complex mediates psoriasis by regulating the expression of complement factor C3. Immunity. 2013 Dec $12 ; 39(6): 1171-81$.

107. He Z, Riva M, Björk P, Swärd K, Mörgelin M, Leanderson T, Ivars F. CD14 Is a CoReceptor for TLR4 in the S100A9-Induced Pro-Inflammatory Response in Monocytes. PLoS One. 2016 May 26;11(5):e0156377.

108. Lessard JC, Piña-Paz S, Rotty JD, Hickerson RP, Kaspar RL, Balmain A, Coulombe PA.Keratin 16 regulates innate immunity in response to epidermal barrier breach. Proc Natl Acad Sci U S A. 2013 Nov 26;110(48):19537-42.

109. Midwood KS, Valenick LV, Hsia HC, Schwarzbauer JE. Coregulation of fibronectin signaling and matrix contraction by tenascin-C and syndecan-4. Mol Biol Cell. 2004 Dec;15(12):5670-7.

110. Chen L, Liu T, Tu Y, Rong D, Cao Y. Cul1 promotes melanoma cell proliferation by promoting DEPTOR degradation and enhancing cap-dependent translation. Oncol Rep. 2016 Feb;35(2):1049-56.

111. Sánchez-Tena S, Cubillos-Rojas M, Schneider T, Rosa JL. Functional and pathological relevance of HERC family proteins: a decade later. Cell Mol Life Sci. 2016 May;73(10):195568.

112. Nakagawa J, Waldner H, Meyer-Monard S, Hofsteenge J, Jenö P, Moroni C. AUH, a gene encoding an AU-specific RNA binding protein with intrinsic enoyl-CoA hydratase activity. Proc Natl Acad Sci U S A. 1995 Mar 14;92(6):2051-5.

113. Chen J, Lu Y, Xu J, Huang Y, Cheng H, Hu G, Luo C, Lou M, Cao G, Xie Y, Ying K. Identification and characterization of NBEAL1, a novel human neurobeachin-like 1 protein gene from fetal brain, which is up regulated in glioma. Brain Res Mol Brain Res. 2004 Jun $18 ; 125(1-2): 147-55$. 
114. Hixson JE, Jun G, Shimmin LC, Wang Y, Yu G, Mao C, Warren AS, Howard TD, Heide RSV, Van Eyk J, Wang Y, Herrington DM. Whole Exome Sequencing to Identify Genetic Variants Associated with Raised Atherosclerotic Lesions in Young Persons. Sci Rep. 2017 Jun $22 ; 7(1): 4091$

115. Tsoi LC, Iyer MK, Stuart PE, Swindell WR, Gudjonsson JE, Tejasvi T, Sarkar MK, Li B, Ding J, Voorhees JJ, Kang HM, Nair RP, Chinnaiyan AM, Abecasis GR, Elder JT. Analysis of long non-coding RNAs highlights tissue-specific expression patterns and epigenetic profiles in normal and psoriatic skin. Genome Biol. 2015 Jan 30;16:24.

116. Yu X, Li Z, Zheng H, Chan MT, Wu WK. NEAT1: A novel cancer-related long noncoding RNA. Cell Prolif. 2017 Apr;50(2). doi: 10.1111/cpr.12329.

117. Kretz M, Siprashvili Z, Chu C, Webster DE, Zehnder A, Qu K, Lee CS, Flockhart RJ, Groff AF, Chow J, Johnston D, Kim GE, Spitale RC, Flynn RA, Zheng GX, Aiyer S, Raj A, Rinn JL, Chang HY, Khavari PA. Control of somatic tissue differentiation by the long noncoding RNA TINCR. Nature. 2013 Jan 10;493(7431):231-5. doi: 10.1038/nature11661.

118. Kretz M. TINCR, staufen1, and cellular differentiation. RNA Biol. 2013 Oct;10(10):1597601. 
Il 
*Szlavicz, $\mathbf{E}^{1,2}$; *Olah, $\mathbf{P}^{2,3}$; Szabo, K ${ }^{1,4}$; Pagani, $\mathrm{F}^{5}$;Bata-Csorgo Zs ${ }^{1,4} ;$ Kemeny, L ${ }^{1,4}$; Szell, $\mathbf{M}^{4,6}$

\title{
Analysis of psoriasis-relevant gene expression and exon usage alterations after silencing of SR-rich splicing regulators
}

\author{
${ }^{1}$ Department of Dermatology and Allergology, Faculty of Medicine, University of Szeged, Szeged, Hungary \\ ${ }^{2}$ Department of Dermatology, Venereology and Oncodermatology, Faculty of Medicine, University of Pécs, \\ Pécs, Hungary \\ ${ }^{3}$ Department of Dermatology, University Hospital Düsseldorf, Düsseldorf, Germany \\ ${ }^{4}$ MTA-SZTE Dermatological Research Group, University of Szeged, Szeged, Hungary \\ ${ }^{5}$ International Centre for Genetic Engineering and Biotechnology, Trieste, Italy \\ ${ }^{6}$ Department of Medical Genetics, Faculty of Medicine, University of Szeged, Szeged, Hungary
}

Word count: 3095

Number of Display items: 4 Figures, 2 Supplementary Figures

* Equal contribution

Corresponding author: Eszter Szlavicz

Email: szlavicz.eszter@gmail.com

Address: Department of Dermatology and Allergology,

University of Szeged

6 Korányi fasor

HU-6720, Szeged, Hungary 


\section{ABSTRACT}

In our recent cDNA microarray experiment, three SR-rich splicing factors - SFRS18, PPIG and $L U C 7 L 3$ - were shown to exert altered responsiveness upon T-lymphokine stimulation of psoriatic non-involved and healthy epidermis samples. We have also demonstrated that double silencing LUC7L3 and SFRS18 efficiently decreased production of the psoriasis-associated EDA+ fibronectin isoform. These findings prompted the further investigation of signaling pathways affected by LUC7L3 and SFRS18.

To detect gene expression and splicing pattern alterations upon double silencing of LUC7L3 and SFRS18 in an HPV-immortalized keratinocyte cell culture, paired-end RNA sequencing was carried out. Marked changes in exon usage were revealed, in contrast to the modest alterations detected in gene expression, providing a closer delineation of the potential targets of the examined splicing factors. The most prominent gene expression change was detected for IFI6, an interferon-inducible gene highly expressed in psoriasis. Interacting partners of IFI6 and certain psoriasis-associated transcripts also exhibited significantly increased expression upon silencing.

In addition to elevated abundance of the EDA+ fibronectin interactor ITGA5, we confirmed decreased EDA-domain inclusion, which agrees well with our prior experimental data. Furthermore, differential exon usage was established for the transcription element CREB1, along with HERC6 and CUL1, which are implicated in ubiquitination. Although immortalized keratinocytes express low levels of TINCR, a long non-coding RNA involved in terminal differentiation of keratinocytes, splicing alterations were successfully demonstrated for this RNA as well.

We believe that the targeted investigation of mRNA maturation disturbances may help us gain deeper insight into the molecular pathogenesis of psoriasis. 


\section{KEYWORDS:}

-RNA Sequencing

-mRNA maturation

-inflammation

-non-coding RNAs

-antiviral immunity 


\section{INTRODUCTION}

Psoriasis is one of the most common chronic inflammatory skin disorders, affecting $2-3 \%$ of the Caucasian population with unquestionable negative impacts on quality of life. Characterization is complicated by the multifactorial origin of the disease, as the interplay of genetic and environmental factors has great influence on the formation of psoriatic lesions. It is well established that innate and adaptive immunological processes both interact in disease pathogenesis ${ }^{[1-3]}$. Recent findings indicate that activation of plasmacytoid dendritic cells could be a determinant step in the initiation phase of psoriasis, which is triggered by the complexes of cathelicidin (LL37) and the self-DNA/RNA of stressed keratinocytes ${ }^{[3-6]}$. This step is followed by the release of type I interferons, as well as the consecutive production of other inflammatory mediators, such as IL-1 $\beta$, IL- 6 and TNF $\alpha$, which are activators of myeloid dermal dendritic cells ${ }^{[3,6,7]}$. This cell type is an important source of IL-12 and IL-23, which are cytokine products that stimulate multiple T-cell lineages implicated in further pathogenic steps, including Th1, Th17 and Th22 lymphocytes ${ }^{[1,3,7,8]}$. Different stages of the disorder are characterized by unique cytokine profiles: whereas the primary phase is dominated by Th1 cells, the propagation of the disorder entails increasing involvement of the Th17 pathway ${ }^{[3,9]}$. Despite the wealth of data, our knowledge regarding the complexity of molecular mechanisms in psoriasis is still incomplete, and further experiments are needed for the detailed description of the molecular background of this inflammatory skin disorder.

In recent years, large-scale gene expression studies have become more accessible and several studies have been conducted to describe gene expression differences of healthy, psoriatic noninvolved and involved epidermis ${ }^{[10-13]}$. The majority of these studies, however, investigated steady-state gene expression levels of various patient-derived samples. In contrast to previous works, we recently investigated T-lymphokine-induced gene expression changes between healthy and psoriatic non-involved epidermis in a cDNA microarray experiment ${ }^{[14]}$. In our experimental setup, organotypic skin cultures were generated, and half of the samples, both healthy and non-involved epidermis, were treated with a lymphokine mixture containing IFN$\gamma$, IL3 and GM-CSF. This lymphokine combination has been shown to facilitate the proliferation of keratinocyte precursors in psoriatic non-involved epidermis; thus, it is likely that these cytokines are important participants of early disorder development ${ }^{[15]}$. 
When comparing T-lymphokine-induced gene expression between healthy and psoriatic epidermis, three SR-rich splicing factors - splicing factor, arginine/serine-rich 18 (SFRS18), peptidyl-prolyl cis-trans isomerase G (PPIG) and luc-7 like protein 3 (LUC7L3) - were found to be differentially regulated in healthy and non-involved psoriatic keratinocytes. The finding is notable, as only a few articles concerning psoriasis and mRNA maturation have been published: we and others have shown that, compared to the healthy samples, EDA+ fibronectin is overexpressed in psoriatic non-involved epidermis and modifies the response of keratinocytes to T-lymphokine stimuli ${ }^{[16-18]}$. In subsequent experiments, we successfully verified that the aforementioned splicing regulators are able to facilitate EDA domain inclusion. Data mining using publicly available interaction databases also supported the credibility of the interaction of LUC7L3 and SFRS18 splicing regulators ${ }^{[19]}$. These data suggest that these splicing factors indeed contribute to disease pathogenesis and their effect is - at least partially -mediated by the regulation of psoriasis-associated EDA+ fibronectin.

In the present study, we aim to determine additional molecular pathways influenced by the SRrich splicing regulators, LUC7L3 and SFRS18, as the combined effects of these two genes proved to be the most potent in prior experiments ${ }^{[19]}$. Global transcriptome analysis was performed using pair-end RNA-Sequencing to define gene expression changes and differential exon usage in HPV-immortalized keratinocytes. 


\section{MATERIALS AND METHODS}

\section{Culturing of HPV-KER cells}

As we described previously, immortalization of the HPV-KER cell line was achieved by the HPV E6 oncogene ${ }^{[20]}$. HPV-immortalized keratinocytes were grown in $75 \mathrm{~cm}^{2}$ cell culture flasks at $37^{\circ} \mathrm{C}$ in a humidified atmosphere containing $5 \% \mathrm{CO}_{2}$. For the maintenance of the cultures, keratinocyte serum-free medium was used (Gibco ${ }^{\circledR}$ Keratinocyte SFM Kit; Life Technologies, Copenhagen, Denmark), supplemented with 1\% L-glutamine (PAA, Pasching, Austria) and $1 \%$ antibiotic/antimycotic solution (PAA). The medium was changed every two days.

\section{Gene-specific silencing}

HPV-KER cells were transiently transfected with gene-specific siRNAs at approximately $70 \%$ confluency. Transfection medium and reagents were ordered from Santa Cruz Biotechnology (Dallas, TX, USA). In our siRNA-mediated silencing experiments, scrambled and gene-specific LUC7L3 and SFRS18 siRNA duplexes were used. The most effective silencing results were obtained in serum-free culture medium without additive and supplements. Real-time RT-PCR was used to confirm the effectiveness of silencing. Quality control of samples submitted to RNA-Seq was carried out by Bioanalyzer (Agilent, Santa Clara, CA, USA), and the measurements indicated excellent RNA quality (RIN: 10).

\section{Sequencing}

Libraries were prepared with cDNA using the Illumina-compatible ScriptSeq RNA-Seq Library Preparation Kit (Epicenter) for 2x100 bp paired-end library construction, for two technical replicates per condition (silenced and control). Sequencing was carried out on the Illumina HiScan SQ platform, resulting in an average depth of $\sim 35$ million raw reads per sample. Library preparation and sequencing were carried out in the Center for Clinical Genomics and Personalized Medicine, University of Debrecen.

\section{Statistical analysis and bioinformatics}


Raw reads in FASTQ format were submitted to quality control using FASTQC, followed by quality and adapter trimming using the fastx toolkit. Filtered reads were mapped to the Hg19 human reference with the STAR aligner, using parameters optimized for splice-site discovery ${ }^{[21]}$. In silico contaminant screening was also carried out, and ambiguously mapping reads were discarded from further analysis. Potential fusion transcripts were predicted by TopHat2 using the fusion-search algorithm ${ }^{[22]}$. Following mapping, transcript assembly and annotation were carried out using Cufflinks, and read counts were summarized at the gene and exon levels by using htseq-count ${ }^{[23,24]}$. Count data were then processed using the DESeq and DEXSeq packages to quantify differential gene expression and differential exon usage, respectively ${ }^{[25]}$. Exons with a coverage $<5$ reads were discarded from the analysis. Functional annotation of differentially expressed genes was carried out using Gene Ontology (GO) enrichment, and extensive comparisons with previous literature-based gene sets and data visualization were carried out in $\mathrm{R}$ version 3.0.1, and Cytoscape.

\section{$\underline{\text { Real-time RT-PCR }}$}

Using the iScript TM cDNA Synthesis kit (\#1708891, Bio-Rad, Hercules, CA), 1 mg total RNA purified from HPV-KER cells was reverse transcribed. Subsequently, real-time RT-PCR was carried out using custom primer sets and the Universal Probe Library (Roche, Basel, Switzerland) with an iQ Supermix (\#1708862, Bio-Rad, Hercules, CA) to quantify transcript abundance. Relative gene expression data were calculated by the $\Delta \Delta \mathrm{Ct}$ method, normalizing the expression to the $18 \mathrm{~S}$ ribosomal RNA.

\section{$\underline{\text { PCR }}$}

Samples were collected from the siRNA silenced HPV-KER cell cultures, and total RNA was isolated using the TRIzol ${ }^{\circledR}$ Reagent (Invitrogen Corp., Carlsbad, CA, USA), according to the instructions of the manual. cDNA synthesis was performed with the iScript cDNA Synthesis Kit (Bio-Rad Laboratories, Hercules, CA, USA) from $1 \mu \mathrm{g}$ total RNA. To amplify a $847 \mathrm{bp}$ product from EDA- fibronectin and a 1221 bp product from EDA+ fibronectin, specific primers were used with PCR conditions summarized in Szell et al. (2004). Semiquantitative analysis of the differentially spliced fibronectin isoforms are also described in the referred paper. Amplification products $(10 \mu \mathrm{l})$ were size fractionated on a $1 \%$ agarose gel at $90 \mathrm{~V}$. Image acquisition and analysis were carried out on a BioRad GelDoc XR densitometer. 


\section{$\underline{\text { RESULTS }}$}

Immortalized HPV-KER cells in which both the LUC7L3 and SFRS18 splicing regulators were silenced were subjected to RNA-Seq profiling. Silencing efficiencies, determined by qPCR prior to sequencing, were between $\sim 70-80 \%$ (Suppl. Fig 1). Following read mapping to reference $\mathrm{Hg} 19$, de novo transcript assembly was performed to identify the potentially novel transcripts and isoforms in the HPV-KER cell line, as well as to provide accurate exon-level annotation for downstream analyses. To assess the alterations caused by the dual silencing of the splicing factors, differential expression and differential exon usage metrics were generated; allowing the complexity and possible errors introduced by isoform reconstruction methods to be circumvented ${ }^{[26,27]}$.

\section{Differential gene expression}

As expected from the experimental model, combined silencing of the LUC7L3 and SFRS18 splicing factors resulted in moderate changes in gene expression (Suppl. Fig. 2), accompanied by extensive disturbances in exon inclusion and exclusion rates. As a result, 35 protein-coding genes were identified as differentially expressed $(\operatorname{logFC}>0.5$, FDR $<0.05)$, with IFI6 and MX1, ISG15 and KRT6A mRNAs showing the most robust fold-changes. Notably, the majority of these regulated genes share functions related to type I interferon signaling based on enrichment analysis. The most significant enriched GO terms were cellular response to type I interferon, cellular response to cytokine stimulus (GO:0071345), regulation of viral genome replication (GO:0045069); these functions were also identified in the functional interaction network based on the Reactome and GeneMania databases (Fig. 1. a). Independent qPCR validation of IFI6 expression indicated up to four-fold changes, which agrees well with our high-throughput results (Fig. 1. b).

\section{$\underline{\text { Differential exon usage }}$}

Differential exon usage was measured for the assembled transcript models, and detected in 224 exons of 217 genes at $\log \mathrm{FC}>0.5$, FDR $<0.1$, corresponding to $\mathrm{p}$-value $<7 \times 10^{-4}$. It is notable that differential usage of multiple exons per gene was detected for a considerable number of 
genes throughout the dataset, possibly indicating fine-tuned changes induced by silencing. Differential exon usage was detected in several non-coding RNAs, whereas functional network analysis of the most significant genes indicted an extensive co-expression background, suggesting overlapping regulation at the transcriptional and post-transcriptional levels (Fig. 2). Transcripts regulated with regard to splicing pattern alterations did not exhibit extensively shared molecular functions, in contrast to regulation detected at the gene expression level. Notably, mining of co-expression-level databases resulted in the identification of abundant relations between regulated genes, indicating their coordinated transcriptional regulation, and, as supported by our current dataset, possibly shared post-transcriptional processing events. The resulting complete functional network contained 172 genes, with only 4 disconnected nodes, and an average node connectivity degree of 13.4. Most connecting edges corresponded to coexpression, followed by physical and genetic interactions. The core set of the most highly connected genes is presented in Fig 3.

Experimental validation of fibronectin exon usage was also carried out on the scrambled controls and siRNA silenced samples of the RNA-Seq experiment. RT-PCR using exonspecific, junction-spanning primers for fibronectin transcripts, followed by visualization with agarose gel electrophoresis, indicated significantly altered inclusion of exon 33 (encoding the EDA domain) and the decreased relative abundance of the EDA+ isoform (Fig. 4). 


\section{DISCUSSION}

In recent years, NGS-based gene-expression profiling techniques, such as RNA-Seq, have revolutionized psoriasis research. RNA-Seq possesses a wider dynamic range compared to microarrays and permits the investigation of potentially novel transcripts, non-coding RNAs and different splicing isoforms ${ }^{[28-33]}$. Nonetheless, only a few psoriasis studies to date address differential exon usage or altered expression of RNA isoforms ${ }^{[30,32,33]}$.

LUC7L3 and SFRS18 splicing factors have been selected based on the results of the aforementioned cDNA microarray experiment, where we identified genes showing $\mathrm{T}$ lymphokine-induced differential expression among control and psoriatic non-involved epidermis ${ }^{[14,19]}$. Products encoded by LUC7L3 and SFRS18 genes are both SR-rich splicing regulators. LUC7L3 - which has a strong sequence similarity with yeast U1 snRNP component Luc7p — has also been shown to participate in the cisplatin-induced resistance of tumor cells ${ }^{[34,35]}$. Another report suggests LUC7L3 protein binding to the cAMP responsive element, and a recent article claimed antiviral property for this splicing regulator [36, 37]. Relatively few data are available for SFRS18. Zimowska and Lin et al. reported interaction of the cell-adhesion and splicing protein pinin (PNN) and SFRS18 ${ }^{[38,39]}$.

The main purpose of the present study was to determine differential gene expression and exon usage upon altered expression of LUC7L3 and SFRS18. In agreement with our primary hypothesis, the extent of gene expression changes was relatively moderate; in contrast, several significant alterations in differential gene expression were induced by the siRNA transfection. Among these data, the most pronounced change was observed for IFI6 (also known as G1P3), an interferon stimulated gene (ISG), which mediates anti-inflammatory, antiviral and antiproliferative effects of interferons. IFI6 is a member of the human ISG12 family, which also includes ISG12A (IFI27), ISG12B, ISG12C. In contrast to other ISG12 isoforms, IFI6 was demonstrated to possess an antiapoptotic feature ${ }^{[40]}$. In addition to its involvement in cancer, biological function of IFI6 in psoriasis pathogenesis has also been reported ${ }^{[40,41]}$. Based on the previous results of our research group, IFI6 might contribute to keratinocyte survival due to its antiapoptotic effect. Experimental data suggest elevated IFI6 gene expression both in the psoriatic non-involved and involved epidermis as compared to the healthy samples ${ }^{[41]}$. Gene expression changes of IFI6 have been successfully validated, exhibiting up to $\sim$ fourfold 
elevation in expression. In addition, we demonstrated increased levels of the ISG12 (IFI27) mRNA, also implicated in psoriasis ${ }^{[42]}$. Thus, LUC7L3/SFRS18 might contribute to balancing pro- and antiapoptotic events.

Furthermore, several predicted interactors for IFI6 (https://stringdb.org/cgi/network.pl?taskId=Mq3bitC7x2Bp) exhibited significant upregulation in the silenced cells. Of these predicted genes, we detected OAS1, OAS2, IFIT1, IFIT3, ISG15, MX1, all of which are related to antiviral immunity ${ }^{[43,44]}$. The elevated expression of MX1, ISG15 and OAS2 in psoriatic skin samples has been verified in a previous experiment ${ }^{[43]}$. In a recent RNA-Seq experiment, Raposo and his co-workers found that 16 antiviral genes - including ISG15 - showed at least twofold elevation in psoriatic epidermis ${ }^{[44]}$. We have also demonstrated LUC7L3/SFRS18-regulated expression of KRT6A and S100A9, which are known innate immunity-related molecules ${ }^{[45,46]}$.

In contrast to our results for differential gene expression, our investigation of differential exon usage indicated more robust alterations. The extracellular matrix protein fibronectin is one of the most suitable models for studying splicing events. Presence of the EDA domain is associated with intrauterine development, wound healing and cancer pathogenesis ${ }^{[47,48]}$. In addition, EDA+ fibronectin was demonstrated to be a putative factor modifying keratinocyte response to mitogenic signals ${ }^{[17]}$. In our previous work, we verified that, similarly to other SRrich proteins (such as SF2/ASF), LUC7L3, PPIG and SFRS18 also facilitate inclusion of the EDA domain ${ }^{[49-51]}$. We were able to validate the significant changes of EDA+/EDAfibronectin abundances in our present large-scale experiment as well ${ }^{[19]}$. In addition to differential splicing of fibronectin, we also demonstrated the significant upregulation of ITGA5, a fibronectin receptor. Data in the literature demonstrate that presence of the EDA domain alters the binding features of fibronectin and increases its affinity for ITGA5 ${ }^{[49,52,53]}$. Elevated levels of ITGA5 might be a compensatory mechanism against the decreased relative amount of EDA+ fibronectin.

LUC7L3, formerly designated CREAP-1, is characterized by its binding domain for the cAMP regulatory element ${ }^{[36]}$. However, in addition to potential direct interaction with CRE, silencing of the splicing factors also alters the splicing pattern of CREB1. Thus LUC7L3 potentially plays a modulatory role in this fundamental element of transcription: altered splicing might influence the protein binding of CREB1. Components of various cellular processes, such as CUL1, HERC1 and HERC6 were are also affected. CUL1 is a component of the E3 ubiquitin ligase 
complex and is overexpressed in melanoma, leading to hyperproliferative processes ${ }^{[54]}$. The HERC gene family is involved in multiple molecular processes, including cell-cycle regulation, DNA repair and ubiquitination, and is also differentially regulated in psoriatic skin. HERC6 is indicated in E3 conjugation of ISG15 ${ }^{[55]}$. OAS2, indicated in antiviral processes, exhibited marked gene expression upregulation as well as differential exon usage. Several genes with relatively sparse functional characterization available in the current literature presented markedly altered exon usage. These include NBEAL1, AUH, with RNA-binding and hydratase activities, and the HERC2 paralog RCC1 ${ }^{[56-58]}$.

Differential exon usage for long non-coding RNAs has been detected using RNA-Seq ${ }^{[31]}$. We have also detected lncRNAs with differential exon usage, most notably TINCR and NEAT1. The role of NEAT1 is relatively well studied in tumorigenesis: its overexpression has been verified in lung, esophagus, colorectal and hepatocellular carcinoma ${ }^{[59]}$. NEAT1 might also be involved in psoriasis, as keratinocyte hyperproliferation is an elementary step in the pathogenesis. The widespread functionality of TINCR lncRNA in keratinocyte maturation has been recently described: whereas another non-coding transcript, ANCR, is needed for the maintenance of keratinocyte precursors, TINCR helps terminal differentiation. In undifferentiated keratinocytes, TINCR is regarded as a low abundance transcript, whereas in differentiated keratinocytes, TINCR exhibited elevated expression ${ }^{[60,61]}$. Accordingly, we have also shown that TINCR is expressed at low levels in HPV-immortalized cells. Altered splicing patterns might affect TINCR binding capacity through the inclusion of one of its several "TINCR box" motifs.

In our present RNA-Seq experiment, we demonstrated that the LUC7L3 and SFRS18 splicing factors contribute to the regulation of several well known psoriasis-associated pathways, including the IFN signaling pathway, antiviral immunity and ubiquitination. Regulatory roles of these SR-rich splicing factors have also been verified for fibronectin mRNA maturation. These results, together with our results for long non-coding RNA expression and exon usage changes, might open new insights to molecular disturbances in early psoriasis development caused by altered mRNA maturation. 


\section{ACKNOWLEDGEMENT}

The work was supported by OTKA grants 5K321 and K105985, and by GINOP-2.3.2-15-201600015 and GINOP-2.2.1-15-2016-00007 research grants. 


\section{REFERENCES}

[1] F. O. Nestle, D. H. Kaplan, J. Barker, N. Engl. J. Med. 2009, 361, 496.

[2] D. Roberson, A. M. Bowcock, Trends. Genet. 2010, 26, 415.

[3] P. Di Meglio, F. Villanova, F. O. Nestle, Cold Spring Harb. Perspect Med. 2014, 4, 8, pii: a015354.

[4] R. Lande, J. Gregorio, V. Facchinetti, B. Chatterjee, Y. H. Wang, B. Homey, W. Cao, Y. H. Wang, B. Su, F. O. Nestle, T. Zal, I. Mellman, J. M. Schröder, Y. J. Liu, M. Gilliet, Nature 2007, 449, 564.

[5] D. Ganguly, G. Chamilos, R. Lande, J. Gregorio, S. Meller, V. Facchinetti, B. Homey, F. J. Barrat, T. Zal, M. Gilliet, J. Exp. Med. 2009, 206, 1983.

[6] A. Farkas, L. Kemeny, Br. J. Dermatol. 2011, 165, 247.

[7] M. A. Lowes, M. Suárez-Fariñas, J. G. Krueger, Annu. Rev. Immunol. 2014, 32, 227.

[8] M. Furue, T. Kadono T, Inflamm. Res. 2017, doi: 10.1007/s00011-017-1065-z.

[9] J. E. Greb, A. M. Goldminz, J. T. Elder, M. G. Lebwohl, D. D. Gladman, J. J. Wu, N. N. Mehta, A. Y. Finlay, A. B. Gottlieb, Nat. Rev. Dis. Primers. 2016, 2, 16082.

[10] E. C. Baechler, F. M. Batliwalla, A. M. Reed, E. J. Peterson, P. M. Gaffney, K. L. Moser, P. K. Gregersen, T. W. Behrens, Immunol. Rev. 2006, 210, 120.

[11] M. Romanowska, N. Yacoub, H. Seidel, S. Donandt, H. Gerken, S. Phillip, N. Haritonova, M. Artuc, S. Schweiger, W. Sterry, J. Foerster, J. Invest. Dermatol. 2008, 128, 110.

[12] JE Gudjonsson, J. Ding, X. Li, T. Tejasvi, Z. S. Qin, D. Ghosh, A. Aphale, D. L. Gumucio, J. J. Voorhees, G. R. Abecasis, J. T. Elder, J. Invest. Dermatol. 2009, 129, 2795.

[13] M. Blumenberg, Curr. Genomics 2012, 13, 363. 
[14] K. Szabo, Z. Bata-Csorgo, A. Dallos, A. Bebes, L. Francziszti, A. Dobozy, L. Kemeny, M. Szell, Acta Derm. Venereol. 2014, 94, 380.

[15] Z. Bata-Csorgo, C. Hammerberg, J. J. Voorhees, K. D. Cooper, J. Clin. Invest. 1995, 95, 317.

[16] K. M. Ting, D. Rothaupt, T. S. McCormick, C. Hammerberg, G. Chen, A. C. Gilliam, S. Stevens, L. Culp, K. D. J. Invest. Dermatol. 2000, 114, 706.

[17] M. Szell, Z. Bata-Csorgo, A. Koreck, A. Pivarcsi, H. Polyanka, C. Szeg, M. Gaal, A. Dobozy, L. Kemeny, J. Invest. Dermatol. 2004, 123, 537.

[18] J. P. McFadden, D. A. Basketter, R. J. Dearman, I. R. Kimber, Clin. Dermatol. 2011, 29, 265.

[19] E. Szlavicz, K. Szabo, G. Groma, Z. Bata-Csorgo, F. Pagani, L. Kemeny, M. Szell, Mol. Cell. Biochem. 2017, doi: 10.1007/s11010-017-3090-1.

[20] H. Polyanka, K. Szabo, G. Tax, V. Tubak, E. Kusz, Z. Ujfaludi, I. Boros, Z. Bata-Csorgo, L. Kemeny, M. Szell, J. Invest. Dermatol. 2011, 131, (Suppl. 2) p. S70.

[21] A. Dobin, C. A. Davis, F. Schlesinger, J. Drenkow, C. Zaleski, S. Jha, P. Batut, M. Chaisson, TR. Gingeras, Bioinformatics 2013, 29, 15.

[22] D. Kim, G. Pertea, C. Trapnell, H. Pimentel, R. Kelley, S. L. Salzberg, Genome Biology 2013, 14, R36

[23] C. Trapnell, B. Williams, G. Pertea, A. Mortazavi, G. Kwan, J. van Baren, S. Salzberg, B. Wold, P. Pachter, Nature Biotechnology 2010, 5, 511.

[24] S. Anders, P. T. Pyl, W, Bioinformatics 2015, 31, 166.

[25] S. Anders, A. Reyes, W. Huber, Genome Research 2012, 22, 2008.

[26] F. Liu, X. Gao, J. Wang, C. Gao, X. Li, X. Li, X. Gong, X. Zeng, J. Mol. Neurosci. 2016, $58,170$.

[27] J. E. Hooper, Human Genomics 2014, 8, 3. 
[28] D. Quigley, J. Invest. Dermatol. 2014, 134, 1789.

[29] A. Jabbari, M. Suárez-Fariñas, S. Dewell, J. G. Krueger, J. Invest. Dermatol. 2012, 132, 246.

[30] B. Li, L. C. Tsoi, W. R. Swindell, J. E. Gudjonsson, T. Tejasvi, A. Johnston, J. Ding, P. E. Stuart, X. Xing, J. J. Kochkodan, J. J. Voorhees, H. M. Kang, R. P. Nair, G. R. Abecasis, J. T. Elder, J. Invest. Dermatol. 2014, 134, 1828.

[31] L. C. Tsoi, M. K. Iyer, P. E. Stuart, W. R. Swindell, J. E. Gudjonsson, T. Tejasvi, M. K.Sarkar, B. Li, J. Ding, J. J. Voorhees, H. M. Kang, R. P. Nair, A. M. Chinnaiyan, G. R. Abecasis, J. T. Elder, Genome Biol. 2015, 16, 24.

[32] M. Keermann, S. Koks, E. Reimann, E. Prans, K. Abram, K. Kingo, BMC Genomics 2015, 16,322 .

[33] S. Koks, M. Keermann, E. Reimann, E. Prans, K. Abram, H. Silm, G. Koks, K. Kingo, Front Med (Lausanne) 2016, 3, 4632.

[34] Y. Nishii, M. Morishima, Y. Kakehi, K. Umehara, N. Kioka, Y. Terano, T. Amachi, K. Ueda, FEBS Lett. 2000, 465, 153.

[35] H. Umehara H, Y. Nishii, M. Morishima, Y. Kakehi, N. Kioka, T. Amachi, J. Koizumi, M. Hagiwara, K. Ueda, Biochem. Biophys. Res. Commun. 2003, 301, 324.

[36] K. L. Shipman, P. J. Robinson, B. R. King, R. Smith, R. C. Nicholson, Biochem. Cell. Biol. 2006, 84,9 .

[37] Y. Li, M. Ito, S. Sun, T. Chida, K. Nakashima, T. Suzuki, Sci. Rep. 2016, 6, 36741.

[38] C. L. Lin, S. Leu, M. C. Lu, P, Biochem. Biophys. Res. Commun. 2004, 321, 638.

[39] G. Zimowska, J. Shi, G. Munguba, M. R. Jackson, R. Alpatov, M.N. Simmons, Y. Shi, S. P. Sugrue, Invest. Ophthalmol. Vis. Sci. 2003, 44, 4715.

[40] H. Gytz, M. F. Hansen, S. Skovbjerg, A. C. Kristensen,S. Hørlyck, M. B. Jensen, M. Fredborg, L. D. Markert, N. A. McMillan, E. I. Christensen, P. M. Martensen, Biol Cell. 2017, $109,94$. 
[41] K. Szegedi , E. Sonkoly, N. Nagy, I. B. Nemeth, Z. Bata-Csorgo, L. Kemeny, A. Dobozy, M. Szell, Exp Dermatol. 2010, 19, 269.

[42] A. M. Bowcock, W. Shannon, F. Du, J. Duncan, K. Cao, K. Aftergut, J. Catier, M. A. Fernandez-Vina, A. Menter, Hum Mol Genet. 2010, 17, 1793

[43] K. Wolk, K. Witte, E. Witte, M. Raftery, G. Kokolakis, S. Philipp, G. Schönrich, K. Warszawska, S. Kirsch, S. Prösch, W. Sterry, H. D. Volk, R. Sabat, Sci Transl Med. 2009, 5, 204

[44] R. A. Raposo, R. Gupta, M. Abdel-Mohsen, M. Dimon, M. Debbaneh, W. Jiang, V. A. York, K. S. Leadabrand, G. Brown, M. Malakouti, S. Arron, P. J. Kuebler, J. J. Wu, S.K. Pillai, D.F. Nixon, W, J Eur Acad Dermatol Venereol 2015, 10, 1951

[45] H. B. Schonthaler, J. Guinea-Viniegra, S. K. Wculek, I. Ruppen, P. Ximénez-Embún, A. Guío-Carrión, R. Navarro, N..Hogg, K. Ashman, E.F. Wagner, Immunity 2013, 39, 1171.

[46] J. C. Lessard, S. Piña-Paz, J.D. Rotty, R. P. Hickerson, R. L. Kaspar, A. Balmain, P. A. Coulombe, Proc Natl Acad Sci USA. 2013, 110, 19537.

[47] E. S. White, A. F. Muro, IUBMB Life 2011, 63, 538

[48] J. Xu, D. Mosher, The Extracellular Matrix: an Overview. 2011, 41.

[49] E. S. White, F. E. Baralle, A. F. Muro, J Pathol, 2008, 1, 14.

[50] M. Caputi, G. Casari, S. Guenzi, R. Tagliabue, A. Sidoli, C. A. Melo, F. E. Baralle, Nucleid Acids Res. 1994 22, 1018.

[51] E. Buratti, A. F. Muro, A. Giombi, D. Gherbassi, A. Iaconcig, F. E. Baralle, Mol Cell Biol. 2004, 24, 1387.

[52] G. Pellegrini, M. De Luca, G. Orecchia, F. Balzac, O. Cremona, P. Savoia, R. Cancedda,P. C. Marchisio, J Clin Invest, 1992, 89, 1783.

[53] K. Y. Okamura, M. Watari, E. S. Jerud, D. W. Young, S. T. Ishizaka, J. Rose, J. C. Chow, J. F. Strauss, J Biol Chem, 2002, 276, 10229. 
[54] L. Chen, T. Liu, Tu Y, D. Rong, Y. Cao, Oncol Rep. 2016, 35, 1049

[55] S. Sánchez-Tena, M. Cubillos-Rojas, T. Schneider, J. L. Rosa, Cell Mol Life Sci. 2016, 73, 1955

[56] J. E. Hixson, G. Jun, L. C. Shimmin, Y. Wang, G. Yu, C. Mao, A. S. Warren, T. D. Howard, R. S. V. Heide, J. Van Eyk, Y. Wang, D. M. Herrington, Sci Rep 2017, 7, 4091

[57] J. Chen, Y. Lu, J. Xu, Y. Huang, H. Cheng, G. Hu, C. Luo, M. Lou, G. Cao, Y. Xie, K. Ying, Mol. Brain Research 2004, 125, 147.

[58] J. Nakagawa, H. Waldner, S. Meyer-Monard, J. Hofsteenge, P. Jeno, C. Moroni, Proc. Natl. Acad. Sci. USA. 1995, 92, 2051.

[59] X. Yu, Z. Li, H. Zheng, M. T. Chan, W. K. Wu, Cell Prolif. 2017, 50(2)

[60] M. Kretz, Z. Siprashvili, C. Chu, D. E. Webster, A. Zehnder, K. Qu, C. S. Lee, R. J. Flockhart, A. F. Groff, J. Chow, D. Johnston, G. E. Kim, R. C. Spitale, R. A. Flynn, G. X. Zheng, S. Aiyer, A. Raj, J. L. Rinn, H. Y. Chang, P. A. Khavari, Nature. 2013, 493, 231.

[61] M. Kretz M, RNA Biol. 2013, 10, 1597. 


\section{FIGURES AND LEGENDS}

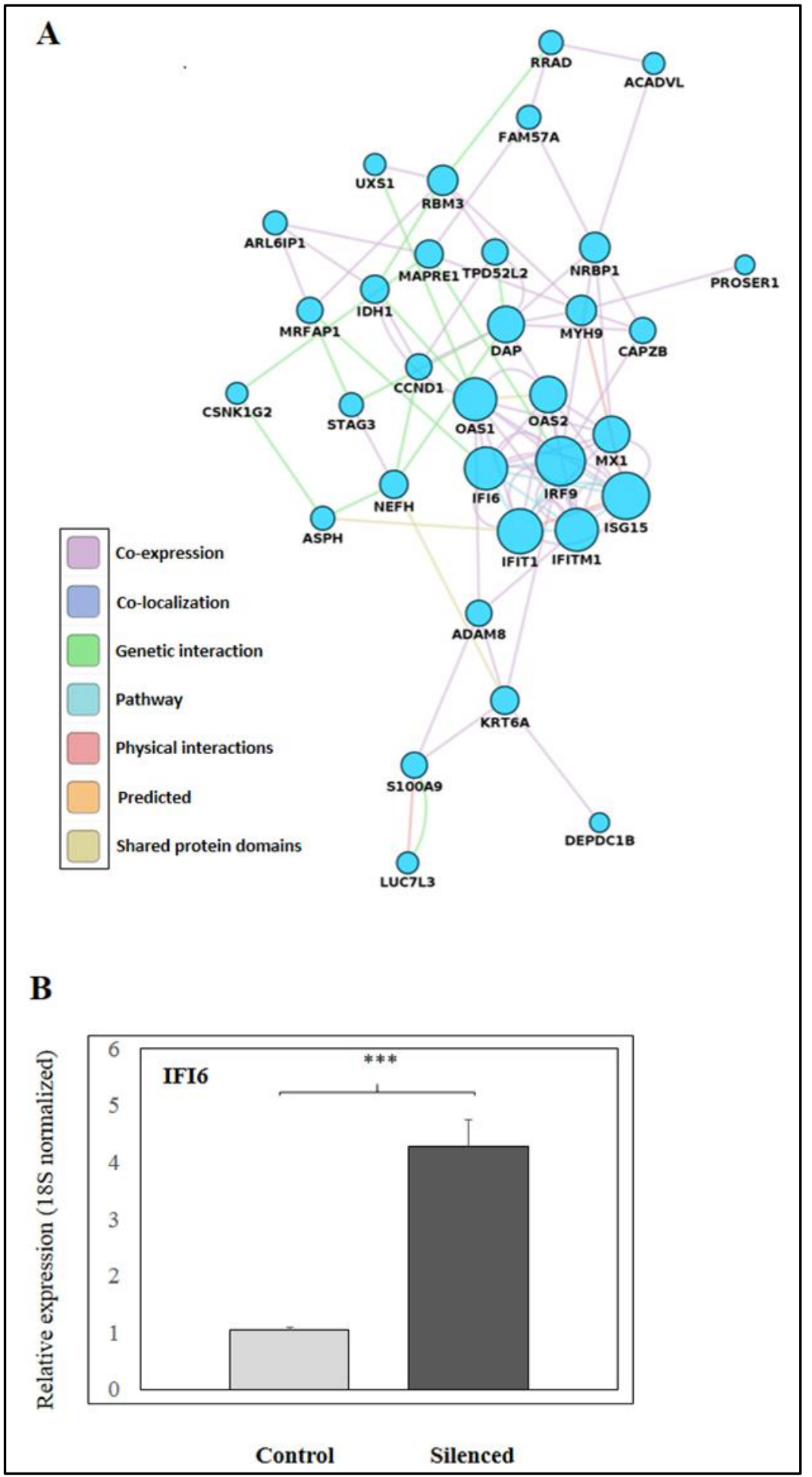

Figure 1: Functional network of significantly differentially expressed genes $(\log 2 \mathrm{FC}>0.5$, FDR $<0.05$ ) in response to dual silencing of LUC7L3 and SFRS18, with edges corresponding to GeneMania and Reactome annotation categories (a). qRT-PCR validation of IFI6 expression 
changes in siRNA-silenced samples; columns present the mean of three independent experiments. $\mathrm{p}<0.05$ was considered as statistically significant (b).

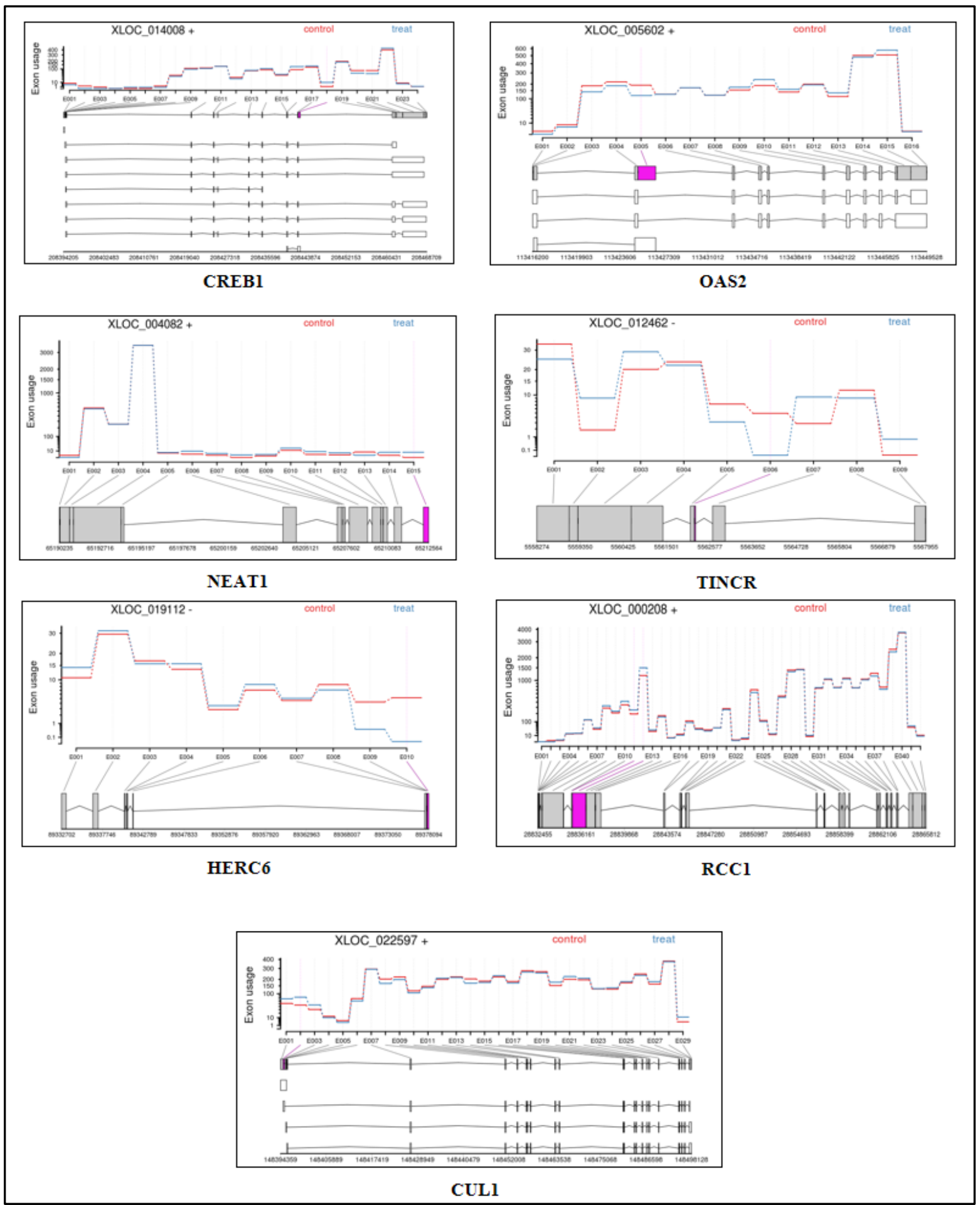

Figure 2: DEXSeq differential-exon-usage plots of selected transcripts showing significant alterations $(\log F C>0.5$, FDR $<0.1)$. Red: average exon usage frequency in control samples; blue: average exon usage frequency in treated samples; pink: significantly differentially expressed exons. De novo assembled transcript diagrams depicted in bottom rows. 


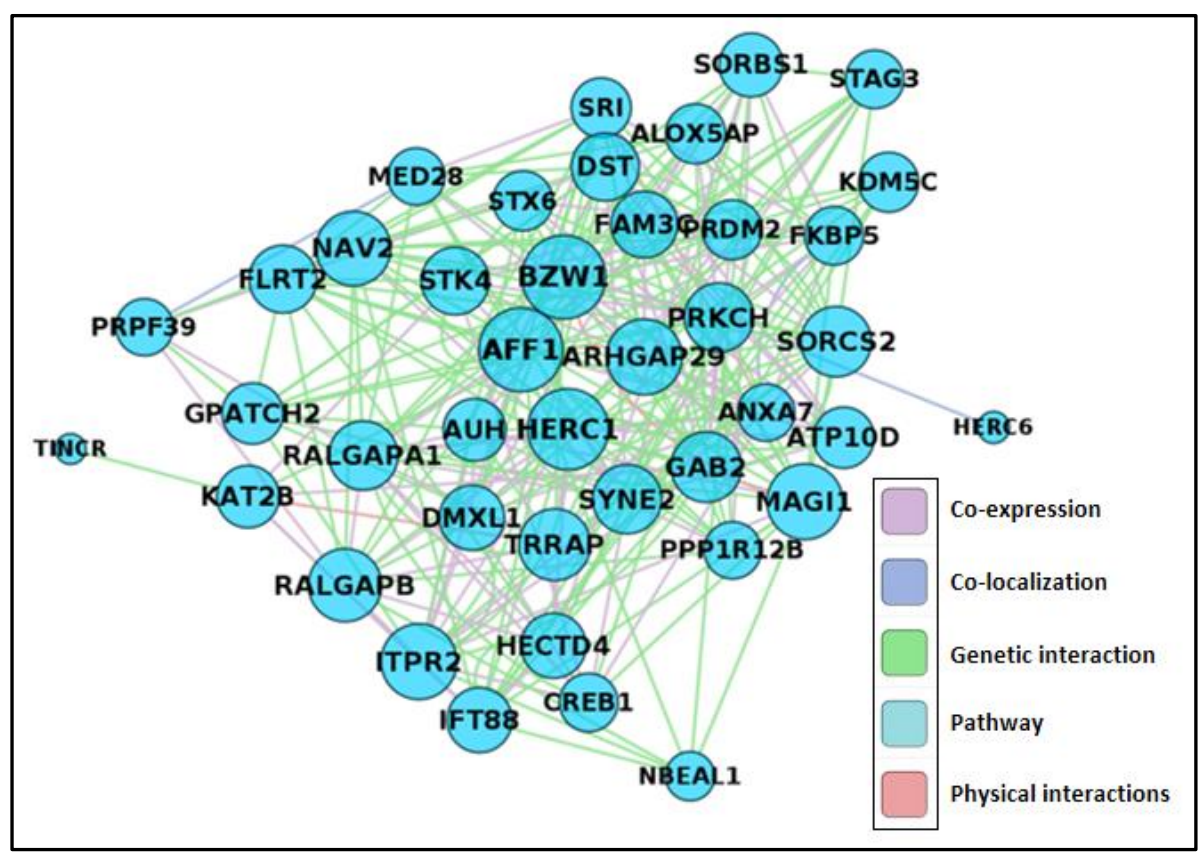

Figure 3: Sub-network representation of a functional network constructed from genes exhibiting significant exon usage changes, with edge color corresponding to GeneMania and Reactome annotation categories. 


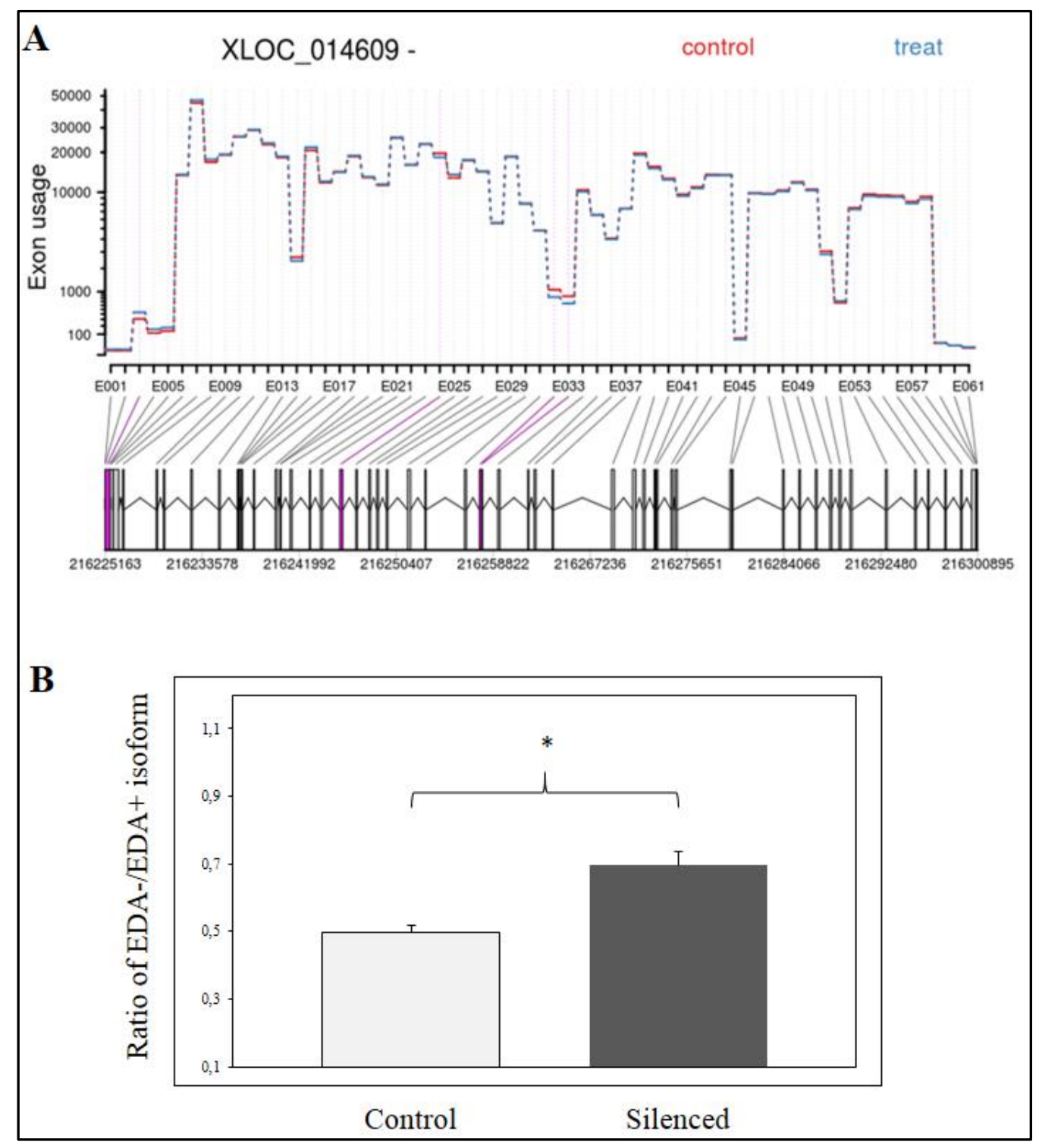

Figure 4: DEXSeq differential-exon-usage plot of fibronectin (FN1), showing the differential inclusion of exon 33, encoding the oncofetal EDA domain. Red indicates the average exonusage frequency in control samples, and blue in silenced samples. Significantly differentially expressed exons are indicated in pink. (a). Independent qRT-PCR-based validation of differential exon usage, indicating abundance of alternative splicing variants in control (grey) and treated (black) samples in double replicates. Significance threshold: $p<0.05$ (b). 


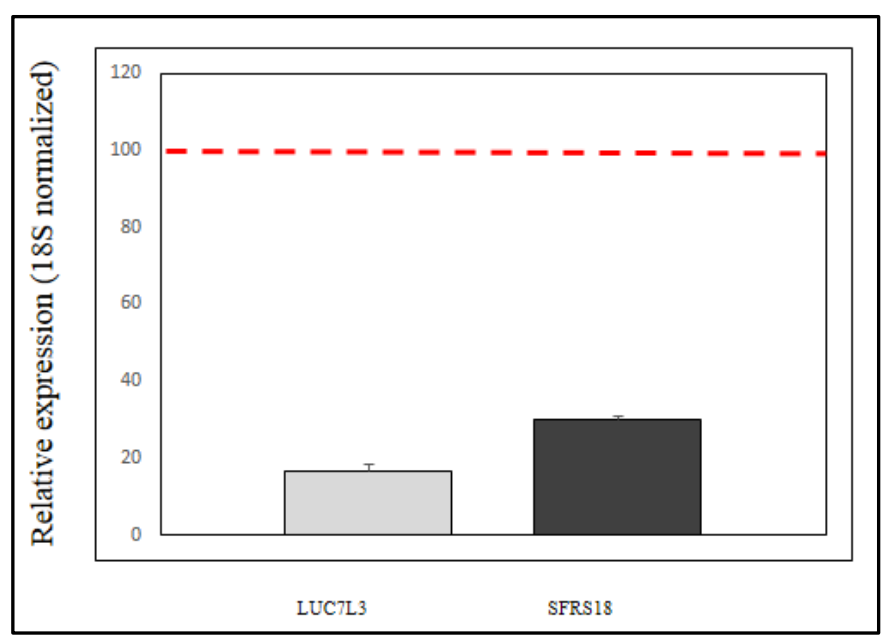

Supplementary Figure 1: Combined siRNA silencing of LUC7L3 and SFRS18 splicing factors was the most effective for the alteration of EDA domain inclusion of fibronectin during mRNA maturation. The efficacy of silencing was $83.6 \% \pm 4.24$ for LUCL73 and $70.1 \% \pm 1,56$ for SFRS18, in two replicates

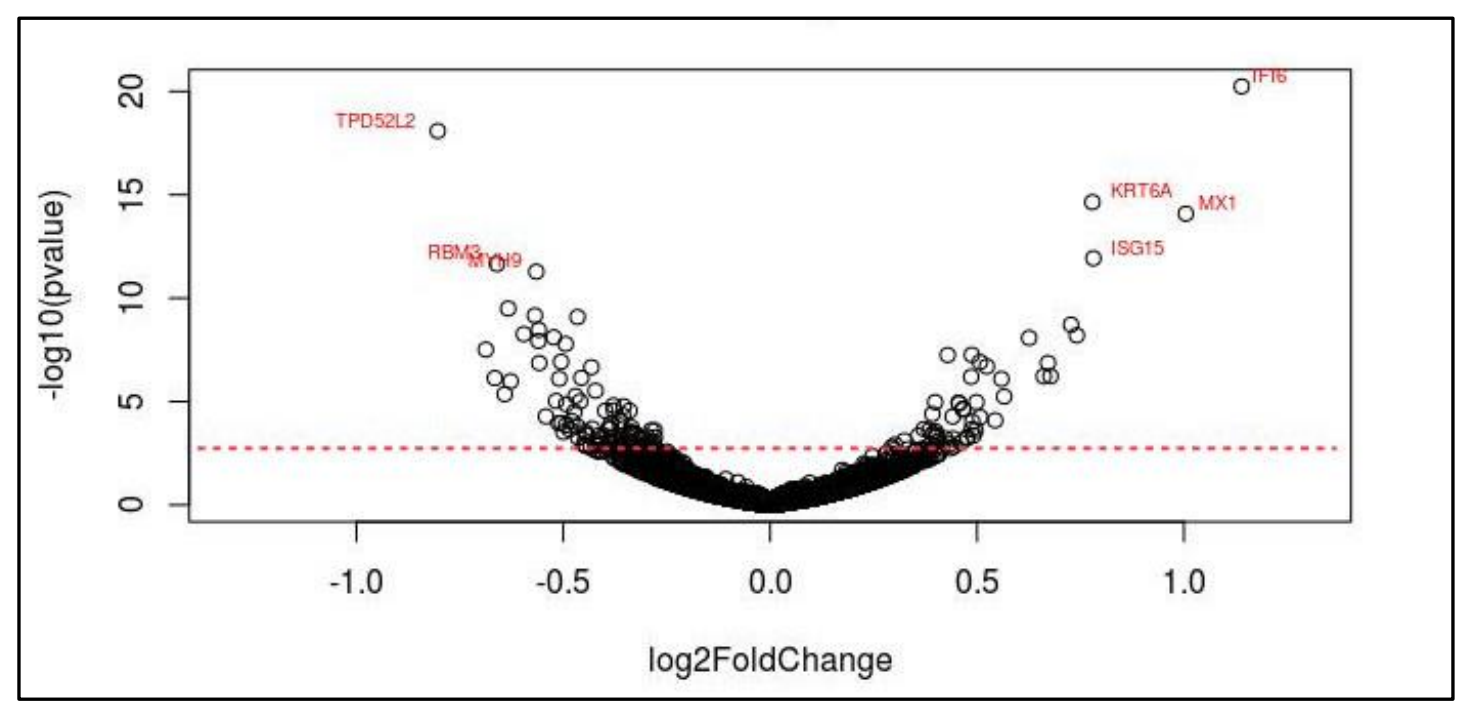

Supplementary Figure 2: Volcano plot of differentially expressed genes upon LUC7L3/SFRS18 double silencing. Dashed line indicates p-value cut-off $=0.001$. 


\begin{tabular}{|c|c|c|c|c|c|c|c|}
\hline Scholarone & anuscripts"m & & & & \begin{tabular}{l|l} 
Eszter Szlavicz V Instr
\end{tabular} & $\begin{array}{ll}\text { tions \& Forms } \mathrm{He} \\
\end{array}$ & \begin{tabular}{l|} 
Help \\
Log Out
\end{tabular} \\
\hline \multicolumn{8}{|c|}{ Experimental Dermatology } \\
\hline * Home & Author & \multicolumn{6}{|c|}{ OReview } \\
\hline \multicolumn{8}{|c|}{ Author Dashboard } \\
\hline \multicolumn{3}{|c|}{ Author Dashboard } & \multicolumn{5}{|c|}{ Submitted Manuscripts } \\
\hline \multicolumn{3}{|c|}{1 submitted Manuscripts > } & StATUS & ID & TITLE & CREATED & SUBMITTED \\
\hline \multirow{2}{*}{\multicolumn{2}{|c|}{$\begin{array}{l}\text { Start New Submission } \\
\text { Legacy Instructions }\end{array}$}} & & \multirow{3}{*}{$\begin{array}{l}\text { ADM: Rodil, Mónica } \\
\text { - Under review }\end{array}$} & \multirow{3}{*}{$\begin{array}{l}\text { EXD- } \\
17- \\
0319\end{array}$} & \multirow{3}{*}{$\begin{array}{l}\text { Analysis of psoriasis-relevant gene expression and exon usage } \\
\text { alterations after silencing of SR-rich splicing regulators } \\
\text { View Submission }\end{array}$} & \multirow[t]{3}{*}{ 19-Sep-2017 } & \multirow[t]{3}{*}{ 20-Sep-2017 } \\
\hline & & & & & & & \\
\hline 5 Most & ecent E-mails & $>$ & & & & & \\
\hline $\begin{array}{l}\text { English } \\
\text { Service }\end{array}$ & anguage Editing & $>$ & & & & & \\
\hline
\end{tabular}




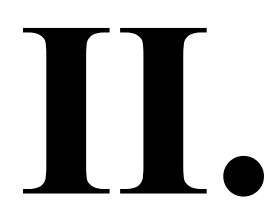




\title{
Splicing factors differentially expressed in psoriasis alter mRNA maturation of disease-associated EDA+ fibronectin
}

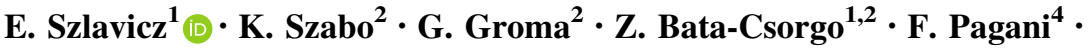 \\ L. Kemeny ${ }^{1,2} \cdot$ M. Szell ${ }^{2,3}$
}

Received: 17 February 2017/ Accepted: 1 June 2017

(C) Springer Science+Business Media, LLC 2017

\begin{abstract}
The EDA+ fibronectin splicing variant is overexpressed in psoriatic non-lesional epidermis and sensitizes keratinocytes to mitogenic signals. However, regulation of its abundance is only partially understood. In our recent cDNA microarray experiment, we identified three SR-rich splicing factors-splicing factor, arginine/serine-rich 18 (SFRS18), peptidyl-prolyl cis-trans isomerase G (PPIG), and luc-7 like protein 3 (LUC7L3)—which might be implicated in the preactivated states of keratinocytes in psoriatic non-involved skin and could also contribute to the regulation of fibronectin mRNA maturation. In this study, we investigated the role of LUC7L3, PPIG, and SFRS18 in psoriasis and in the mRNA maturation process of fibronectin. Regarding tissue staining experiments, we were able to demonstrate a characteristic distribution of the splicing factors in healthy, psoriatic non-involved and involved epidermis. Moreover, the expression profiles of these SR-rich proteins were found to be very similar in synchronized keratinocytes. Contribution of splicing
\end{abstract}

Electronic supplementary material The online version of this article (doi:10.1007/s11010-017-3090-1) contains supplementary material, which is available to authorized users.

E. Szlavicz

szlavicz.eszter@gmail.com

1 Department of Dermatology and Allergology, Faculty of Medicine, University of Szeged, 6 Korányi fasor, Szeged 6720, Hungary

2 MTA-SZTE Dermatological Research Group, Szeged, Hungary

3 Department of Medical Genetics, Faculty of Medicine, University of Szeged, Szeged, Hungary

4 International Centre for Genetic Engineering and Biotechnology, Trieste, Italy facwwtors to the EDA+ fibronectin formation was also confirmed: their siRNA silencing leads to altered fibronectin mRNA and protein expression patterns, suggesting the participation in the EDA domain inclusion. Our results indicate that LUC7L3, PPIG, and SFRS18 are not only implicated in EDA+ fibronectin formation, but also that they could possess multiple roles in psoriasis-associated molecular abnormalities.

Keywords Psoriasis - mRNA splicing · EDA+ fibronectin $\cdot$ SR-rich splicing factors

\section{Introduction}

During the past few years, huge advances have been made in the field of psoriasis research; however, the exact molecular background of this chronic inflammatory disorder has not been fully revealed and many questions remain to be answered. Development of psoriatic lesions is associated with keratinocyte hyperproliferation and abnormal T-lymphocyte function, although it is still controversial whether epithelial or immunological elements are determinant in the primary phase of the disease. The extent to which genetic and environmental factors lead to the disorder is also a subject of discussion [1-3].

Recently, a novel molecular factor has been identified that might be responsible for the elevated responsiveness of keratinocytes to the stimuli of professional immune cells. This putative factor is a splice variant of fibronectin that contains the oncofetal EDA domain (EDA+ fibronectin) [4-6]. Although fibronectin is an excellent model for studying splicing (having more than 20 mRNA variants), processes regulating mRNA maturation and the mechanisms affecting EDA+ fibronectin formation are not fully 
understood [7, 8]. The EDA domain is a Type III repetitive module of fibronectin, typically present during embryonic development. Although the tendency is exclusion of the EDA domain in the adult organism, EDA+ fibronectin is elevated under some circumstances, including wound healing, inflammation, and malignant processes [8-13]. In psoriasis, elevated EDA+ fibronectin expression was described for psoriatic non-involved epidermis, and we showed that proliferating keratinocytes are able to produce this isoform $[4,5]$.

Several molecular factors in addition to EDA+ fibronectin have been implicated in psoriasis pathogenesis. Application of large-scale gene expression studies extended our knowledge regarding molecular abnormalities in involved and non-involved epidermis [14-19]. Recent RNASequencing-based experiments have indicated that a large number of mRNAs are consistently differentially expressed in healthy and involved skin samples across independent studies, at both the transcript and gene levels [20, 21]. Specifically, a systematic comparison of the above-mentioned datasets indicated over 10,000 differentially expressed transcripts, suggesting the potential role of mRNA maturation processes in psoriasis development [22].

In a recently performed cDNA microarray experiment, we aimed to compare the responsiveness of healthy and psoriatic non-involved epidermis to treatment with a mixture of T-lymphokines (GM-CSF, IFN- $\gamma$, IL-3) previously shown to induce psoriatic plaque formation [23, 24]. Functional characterization of the identified genes exhibiting differential regulation upon T-lymphokine treatment showed that they play a role in several cellular procedures including the influence of cell morphology, development, and cell death. Moreover, a set of these genes participate in the metabolism of small lipids [23]. Among these genes were three that are implicated in mRNA maturation: splicing factor, arginine/serine-rich 18 (SFRS18, also known as PNN-interacting serine/arginine-rich protein, PNISR), peptidyl-prolyl cis-trans isomerase G (PPIG), and luc-7 like protein 3 (LUC7L3). Products of these genes belong to the family of SR-rich proteins and function as trans-regulators of splicing events (UniProtKBO95232 (LC7L3_HUMAN); UniProtKB-Q13427 (PPIG_HUMAN); UniProtKB-Q8TF01 (PNISR_HUMAN)). This finding is notable, as relatively few articles mention the relationship between psoriasis and disturbances of mRNA maturation.

In our study, characteristics of splicing-factor expression were examined in synchronized, immortalized cell cultures and skin samples. Since the regulation of fibronectin splicing is only partially elucidated, we aimed to determine whether these newly identified splicing factors participate in the production of the EDA+ fibronectin isoform. For this purpose, an in vitro functional assay was constructed to detect changes in fibronectin splicing patterns during decreased expression of the LUC7L3, PPIG, and SFRS18 splicing factors.

\section{Materials and methods}

\section{Skin biopsies}

To carry out our cDNA microarray experiment, 4 healthy volunteers and 4 patients (age 18-60 years) with moderateto-severe chronic plaque-type psoriasis (Psoriasis Area and Severity Index score determined by dermatologists) were enrolled in the study after a medication-free period of $\geq 4$ weeks without systemic therapy and/or $\geq 2$ weeks without local therapy. Prior to surgical intervention, participant-informed consent was obtained. Tissue collection complied with the guidelines of the Declaration of Helsinki and was approved by the Regional and Institutional Research Ethics Committee (2799, 3517).

\section{Organotypic skin cultures}

Organotypic skin cultures were established from each halfcut from shave biopsies [23]. Briefly, epidermis samples placed on cellulose acetate/cellulose nitrate filters $(2.2 \mu \mathrm{m}$ porosity, Millipore) were transferred to a stainless steel grid platform in a 6-well plate. As culture medium, Dulbecco's modified Eagle's medium (DMEM) supplemented with $12 \mathrm{mM}$ glutamine, 100 units $/ \mathrm{ml}$ penicillin, and $100 \mu \mathrm{g} / \mathrm{ml}$ streptomycin (all from Life Technologies, Carlsbad, CA) was applied. For all donor skin specimens, one-half of the organotypic cultures was treated with the lymphokine mixture, containing $1 \mathrm{ng} / \mathrm{ml} \mathrm{IFN} \gamma, 1 \mathrm{ng} / \mathrm{ml}$

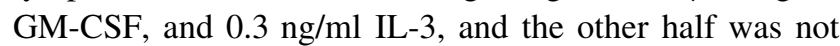
treated. Using standard culturing conditions (at $37{ }^{\circ} \mathrm{C}$ in a $5 \% \mathrm{CO}_{2}$ atmosphere), organotypic skin cultures were maintained at the air/liquid interface for $72 \mathrm{~h}$. Subsequently, the epidermis was separated from the dermis by overnight incubation in Dispase solution (grade II, Roche Applied Science) at $4{ }^{\circ} \mathrm{C}$, and then placed in TRI Reagent (Molecular Research Center Inc., Cincinnati, OH).

\section{Real-time RT-PCR}

One microgram of total RNA purified from organotypic skin culture samples, HPV-KER and HaCaT cells were reverse transcribed using the iScript TM cDNA Synthesis kit (\#1708891, Bio-Rad, Hercules, CA). RT-PCR was performed with the cDNA to quantify transcript abundance using custom primer sets and the Universal Probe Library (Roche, Basel, Switzerland) with an iQ Supermix (\#1708862, Bio-Rad, Hercules, CA). Relative gene expression data were calculated by normalizing the 
expression data for the $18 \mathrm{~S}$ ribosomal RNA and using the $\Delta \Delta \mathrm{Ct}$ method.

\section{Immunofluorescent staining}

Biopsies were frozen, embedded, stored at $-80{ }^{\circ} \mathrm{C}$, and then cut into $6-\mu \mathrm{m}$ sections. The following primary antibodies were used: LUC7L3 (1:300, Abcam, Cambridge, UK), PPIG (1:300, Abcam), and SFRS18 (1:250, Novus Biologicals, Littleton, USA). Anti-mouse IgG-Alexa Fluor 647 and anti-rabbit Alexa Fluor 546 were used as secondary antibodies, both at 1:500 dilution (Life Technologies, Carlsbad, CA). For negative staining controls, sections were incubated without the primary antibody (in case of PPIG) or with normal rabbit IgG as isotype control antibody (Santa Cruz Biotechnology, Dallas, TX, USA). Nuclei were stained with 2-(4-amidinophenyl)-1H-indole6-carboxamidine (DAPI).

Fluorescent microscopic images were analyzed using ImageJ software, by calculating corrected-total-cell-fluorescence values based on integrated density of stained cell areas.

\section{Culturing of HPV-KER and HaCaT cells}

The HPV-KER cell line was immortalized by the HPV E6 oncogene as described by our research group [25]. HPVKER cells were grown in $75 \mathrm{~cm}^{2}$ cell culture flasks and maintained in keratinocyte serum-free medium $\left(\mathrm{Gibco}^{\circledR}\right.$ Keratinocyte SFM Kit; Life Technologies, Copenhagen, Denmark) supplemented with $1 \%$ antibiotic/antimycotic solution (PAA, Pasching, Austria) and 1\% L-glutamine (PAA) at $37{ }^{\circ} \mathrm{C}$ in a humidified atmosphere containing $5 \%$ $\mathrm{CO}_{2}$. The medium was changed every two days.

During synchronization of the HPV-KER cells and after confluence was obtained, cells were maintained in glutamine-free and growth factor-free keratinocyte SFM for one week. Cells were harvested with brief trypsinization $(0.25 \%$ trypsin solution Sigma Aldrich, St. Louis, MO, USA)

Immortalized HaCaT keratinocytes were kindly provided by Dr. Fusenig, N.E. (Heidelberg, Germany). Cells were synchronized as described previously [26].

\section{Western blot analysis}

For western blot analysis, equal amounts of proteins were separated on a $10 \%$ SDS-PAGE gel and transferred to Pure Nitrocellulose Membrane (Bio-Rad Laboratories). Primary antibodies were the following: LUC7L3 (1:300, Abcam, Cambridge, UK), PPIG (1:300, Abcam), and SFRS18 (1:300, Novus Biologicals, Littleton, US). Anti-mouse and anti-rabbit IgG alkaline phosphate conjugate (Sigma Aldrich, St. Louis, MO, USA) was used as a secondary antibody, and signals were visualized with Sigma Fast TM BCIP/NBT (Sigma Aldrich, St. Louis, MO, USA). An $\alpha$ actin-specific antibody was used as a loading control (1:2000, Sigma Aldrich, St. Louis, MO, USA).

\section{Gene-specific silencing}

Gene-specific silencing was performed with an siRNA transfection method. HPV-KER cells were transiently transfected at approximately $70 \%$ confluency. siRNA silencing (transfection reagent and medium) was ordered from Santa Cruz Biotechnology (Dallas, TX, USA). In silencing experiments, scrambled and gene-specific LUC7L3, PPIG, SFRS18 siRNA duplexes were applied. The most effective silencing was achieved in serum-free culture medium without additive and supplements. The effectiveness of silencing was confirmed by real-time RT-PCR.

\section{Polymerase chain reaction}

Samples were collected from the cultures of silenced HPVKER cells. Total RNA was isolated from cell cultures by TRIzol ${ }^{\circledR}$ Reagent (Invitrogen Corp., Carlsbad, CA, USA), following the instructions provided in the manual. cDNA was synthesized from $1 \mu \mathrm{g}$ total RNA with the iScript cDNA Synthesis Kit (Bio-Rad Laboratories, Hercules, CA, USA). Primers specific for human fibronectin (5'-AAGCCAATTTCCATTAATTACCGAAC- $3^{\prime}$ and 5'-TCTCATACT TGATGATGTAGCCGGTAA- $3^{\prime}$ ) were used to amplify a $1221 \mathrm{bp}$ product from the EDA+ template and an 847 bp product from the EDA - fibronectin template. PCR conditions are described in Szell et al., and detection and semiquantitative analysis of the differentially spliced fibronectin form are also demonstrated in this paper [4]. Ten microliters of the PCR products were run on $1 \%$ agarose gel, photographed and evaluated using Bio-Rad Gel Doc XR densitometer.

\section{Flow cytometry}

Cells were collected after a brief trypsinization $(0.25 \%$ trypsin solution Sigma Aldrich, St. Louis, MO, USA), fixed in Fixation/Permeabilization Concentrate and Diluent (eBioscience, San Diego, USA), and resuspended in PBS. Primary antibodies (anti-EDA+-fibronectin, anti-fibronectin, 1:500 and 1:1000, respectively, Sigma Aldrich, St. Louis, MO, USA) were applied for $45 \mathrm{~min}$. Cells were then washed in PBS and incubated with anti-mouse IgGAlexa Fluor 647 (Life Technologies, Carlsbad, CA) as secondary antibody, at 1:500 dilution. Samples were analyzed on a FACSCalibur flow cytometer equipped with 488 and 633 nm lasers (Becton-Dickinson, Franklin Lakes, NJ, USA). 


\section{Statistical analysis and bioinformatics}

Statistical analysis was performed in GraphPad Prism 5.0 Software (GraphPad Prism, San Diego, CA), using onesample $t$ - and unpaired $t$-tests with a $p<0.05$ significance threshold.

Predictive interaction analysis was carried out using the publicly available STRING database (http://string-db.org/).

\section{Results}

\section{Differential expression of splicing-factor genes confirmed for psoriasis}

LUC7L3, PPIG, and SFRS18 splicing regulators have been identified in our above-mentioned cDNA microarray experiment. An overview of our approach is presented in Fig. 1.a. Briefly, gene expression before and after lymphokine treatment was compared in healthy epidermis, and we applied the same approach to non-involved psoriatic skin. Genes exhibiting at least a two-fold change in relative expression have been chosen for further comparison. We then selected the genes showing significant differences in mean expression between the control and psoriatic noninvolved samples [23]. Real-time reverse-transcription polymerase chain reaction (RT-PCR) was carried out to verify whether the identified genes are differentially regulated in psoriasis. The T-lymphokine induced alterations were confirmed in the case of both LUC7L3 and PPIG splicing factors (Fig. 1b) Although we were not able to validate the cDNA microarray results for SFRS18 in the RT-PCR experiment, we decided to include this gene in further experiments as it participates in similar biological processes as $L U C 7 L 3$ and $P P I G$.

Expression of the splicing factors was also compared in untreated healthy and psoriatic non-involved epidermis samples of the microarray experiment: in this setup, the expression of LUC7L3 and SFRS18 was slightly higher in psoriatic non-involved epidermis than in healthy samples (Fig. 1c).

\section{Differentially expressed splicing factors in psoriasis}

As the splicing factors showing altered mRNA expression in psoriasis are poorly characterized, we examined the pattern of protein expression in healthy, psoriatic non-involved and involved skin samples. LUC7L3 and PPIG exhibited nuclear localization, whereas SFRS18 showed perinuclear staining (Fig. 2a). LUC7L3 is present in significantly higher amounts in psoriatic non-involved
Fig. 1 Experimental design and comparisons used in our previous cDNA microarray study is summarized in (a). Gene expression changes of LUC7L3, PPIG, and SFRS18 splicing factors induced by T-lymphokine treatment were compared in healthy $(\mathrm{H}, n=4)$ and psoriatic non-involved (PS, $n=4)$ epidermis samples (b), where the average expression level of untreated samples was considered as baseline expression (relative unit of 1 indicated by dashed line). Comparison of $L U C 7 L 3, P P I G$, and SFRS18 basal mRNA expression levels in untreated healthy $(\mathrm{H}, n=4)$ and psoriatic non-involved (PS, $n=4)$ epidermis. $p<0.05$ was considered significant $(\mathbf{c})$ epidermis, whereas SFRS18 shows only minimal, nonsignificant elevation in non-involved epidermis. The highest levels of expression of LUC7L3 and SFRS18 were found in psoriatic involved epidermis samples. The expression pattern of PPIG was different from that of the other splicing factors: PPIG amounts were significantly lower in psoriatic non-involved epidermis than in healthy and psoriatic samples; however-similarly to LUC7L3 and SFRS18 - the highest expression was also detected in psoriatic involved epidermis (Fig. 2b).

\section{LUC7L3, PPIG, and SFRS18 expression patterns in synchronized cells}

To compare the expression levels in proliferating and differentiating keratinocytes, both mRNA and protein expression patterns of the selected splicing factors were analyzed in synchronized, immortalized cells. HPV-KER cells were synchronized by contact inhibition and in the absence of growth factors [22]. Cells were harvested at various time points after release from cell quiescence, and RNA and protein levels were measured by real-time RTPCR and western blot analysis, respectively.

Comparing the expression of the splicing factors during the proliferation and differentiation phases, a very similar mRNA expression pattern was observed for all three splicing factors (Fig. 3a). mRNA expression decreased at $12 \mathrm{~h}$. The first peak of mRNA abundance was seen at $24 \mathrm{~h}$, with steady elevation starting from $48 \mathrm{~h}$, a phenomenon that is likely induced by cellular stress processes. This synchronization experiment was repeated using synchronized, immortalized $\mathrm{HaCaT}$ cells: the splicing regulators exhibited an identical pattern of gene expression in $\mathrm{HaCaT}$ cells that were observed in the HPV-keratinocytes (Suppl. Fig. 1)

The pattern of expression in HPV-KER cells of both RNA and protein was similar. However, protein expression was more even across sampled time points than for mRNA. The highest expression of LUC7L3, PPIG, and SFRS18 protein was detected at $48 \mathrm{~h}$ after release from cell quiescence (Fig. 3b). 
(A)
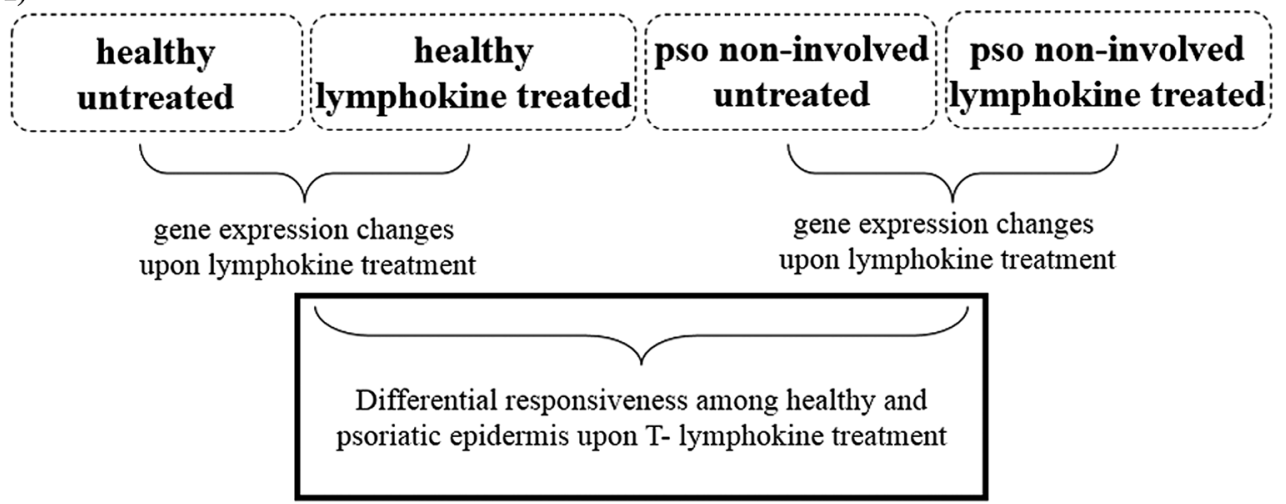

Fold-change $>2, p<0.05,95 \% \mathrm{CI}$

(B)
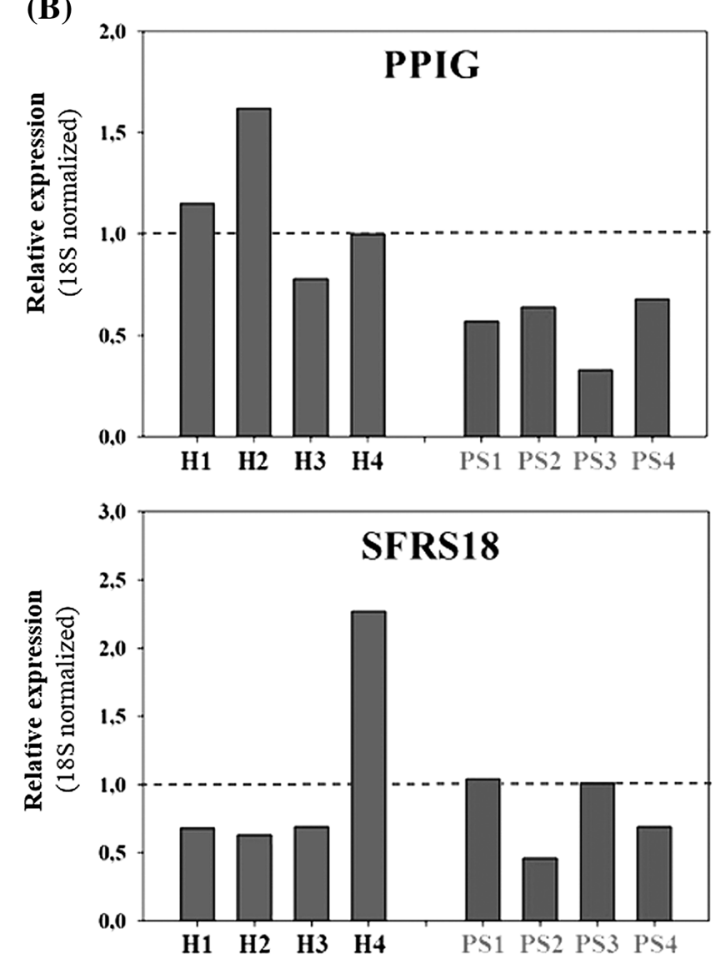

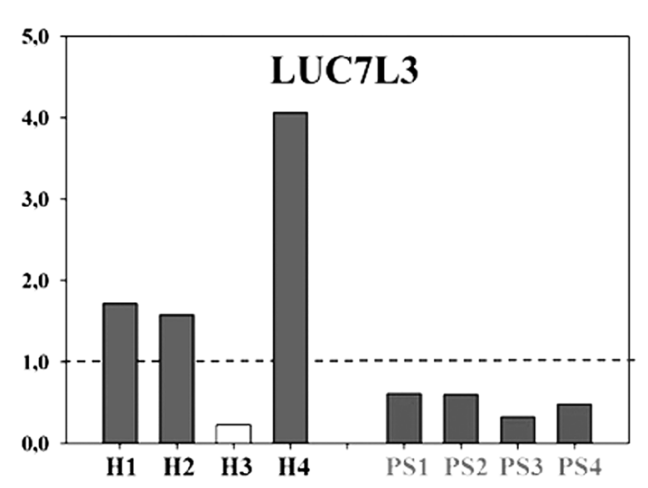

(C)

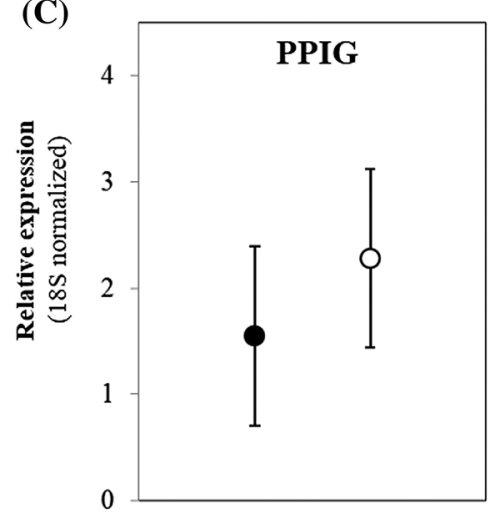

H PS

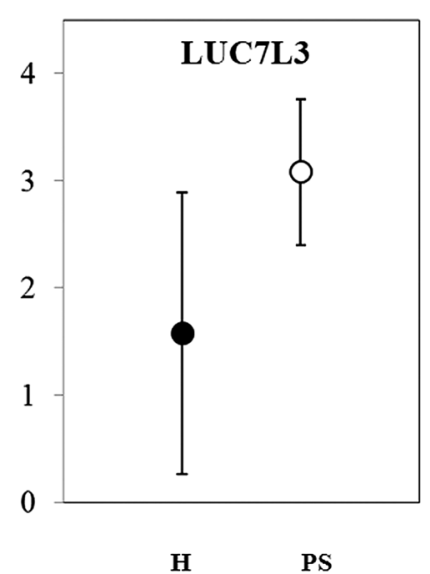

H

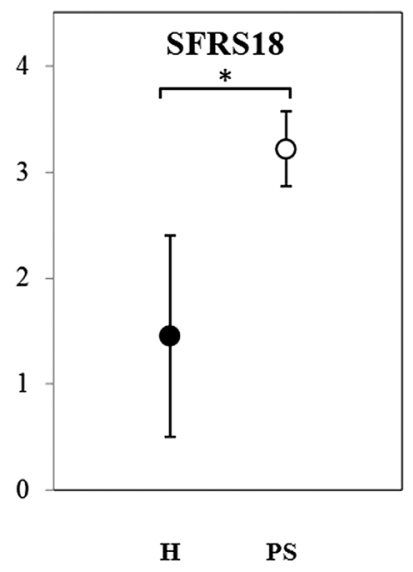



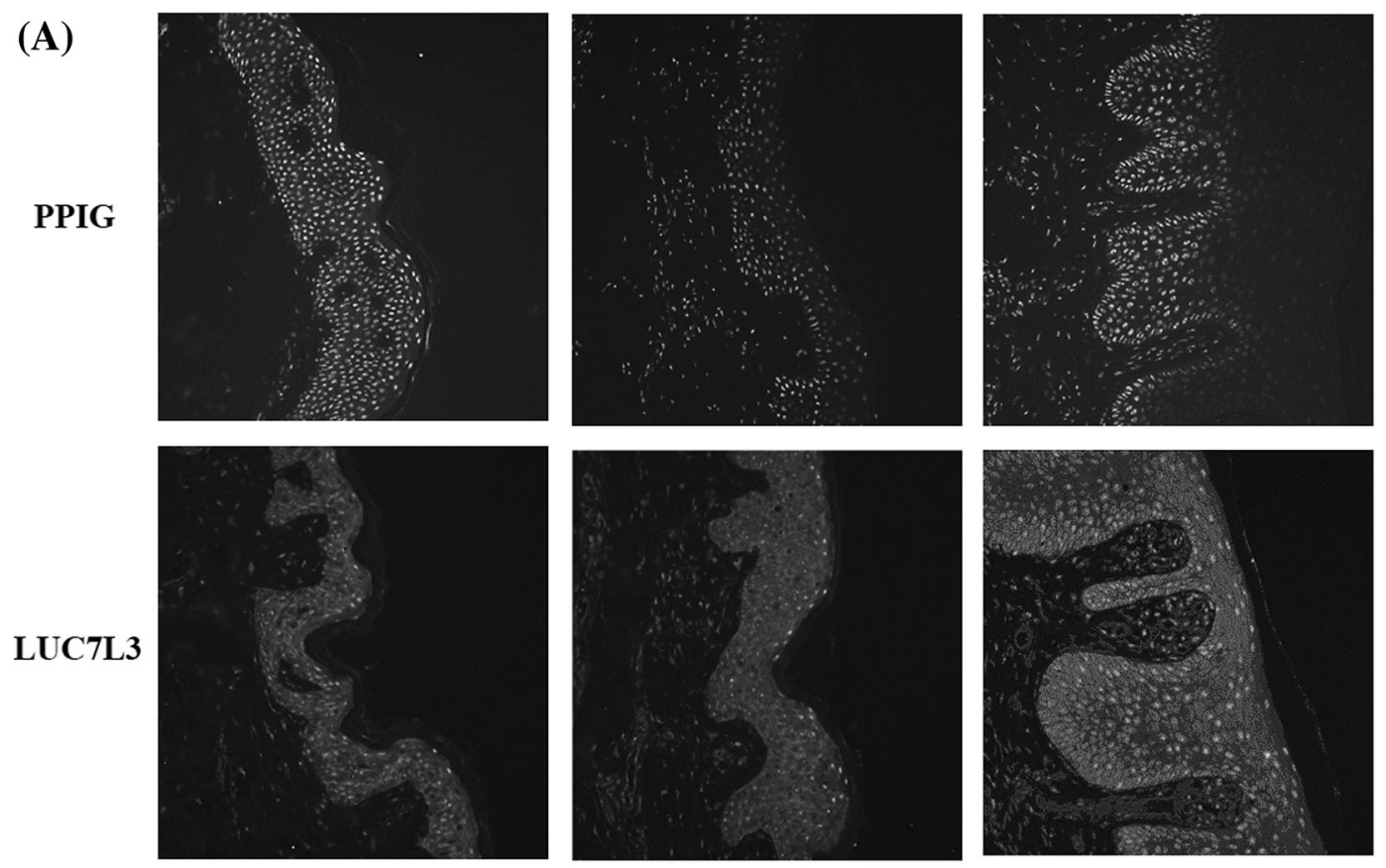

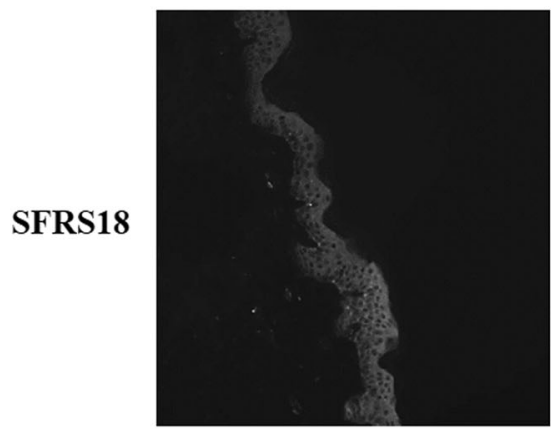

Healthy epidermis

(B)

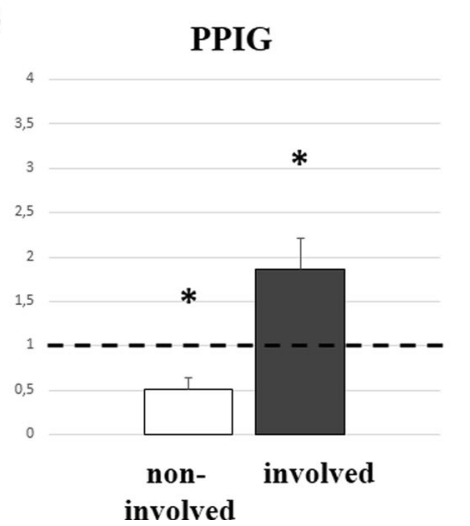

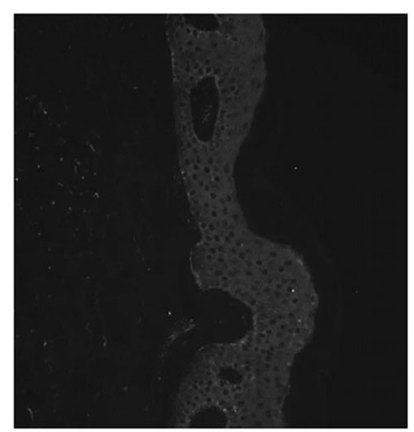

\section{Psoriatic non-involved} epidermis

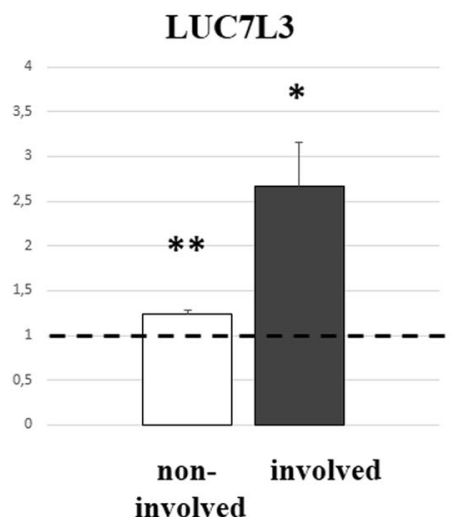

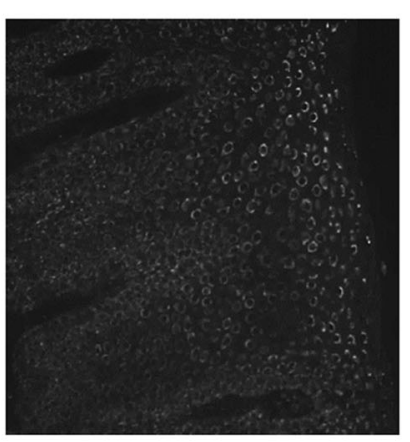

Psoriatic involved epidermis

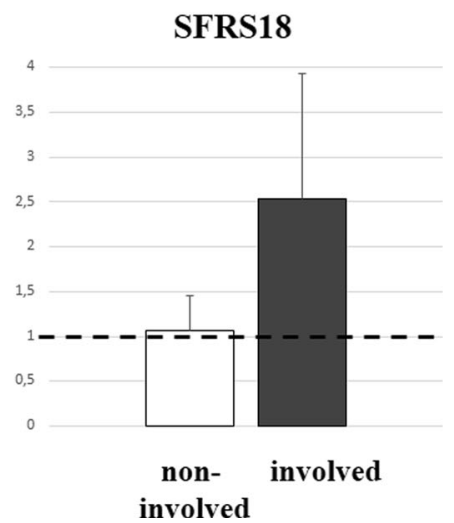

Fig. 2 Immunofluorescent staining of PPIG, LUC7L3, and SFRS18 proteins (a) was carried out on healthy $(n=3)$, psoriatic noninvolved $(n=3)$, and psoriatic involved $(n=3)$ samples. One representative staining is provided for each protein. Magnification: $\times 40$. Fluorescent intensity measurements of PPIG, LUC7L3, and

SFRS18 (b) in non-involved and involved epidermis compared to healthy samples are indicated as follows: dashed lines represent the unaltered condition, where the ratio of non-involved/healthy and involved/healthy is 1; significance levels are indicated by asterisks $(* p<0.05, * * p<0.01, * * * p<0.001)$ 

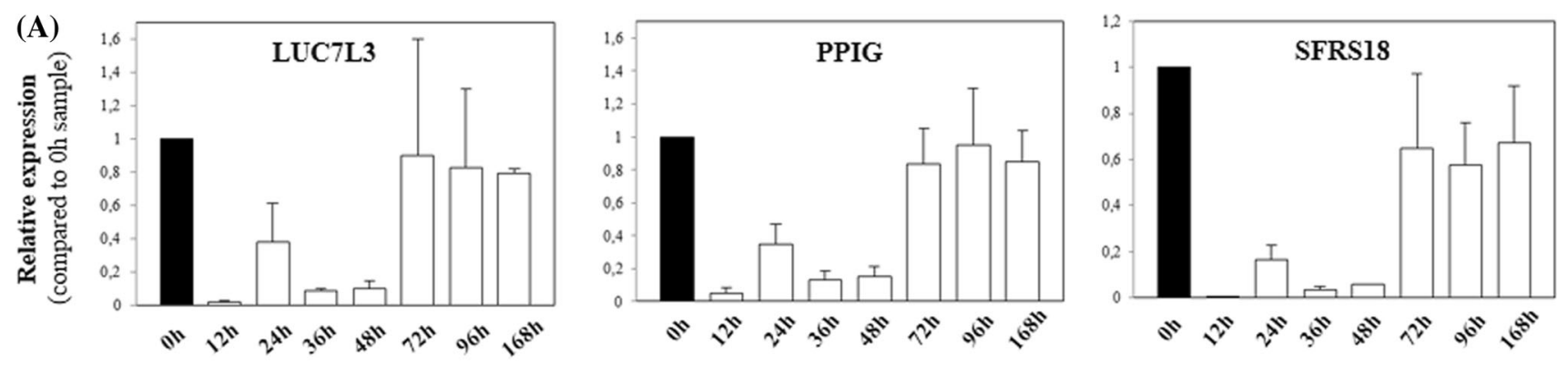

(B)
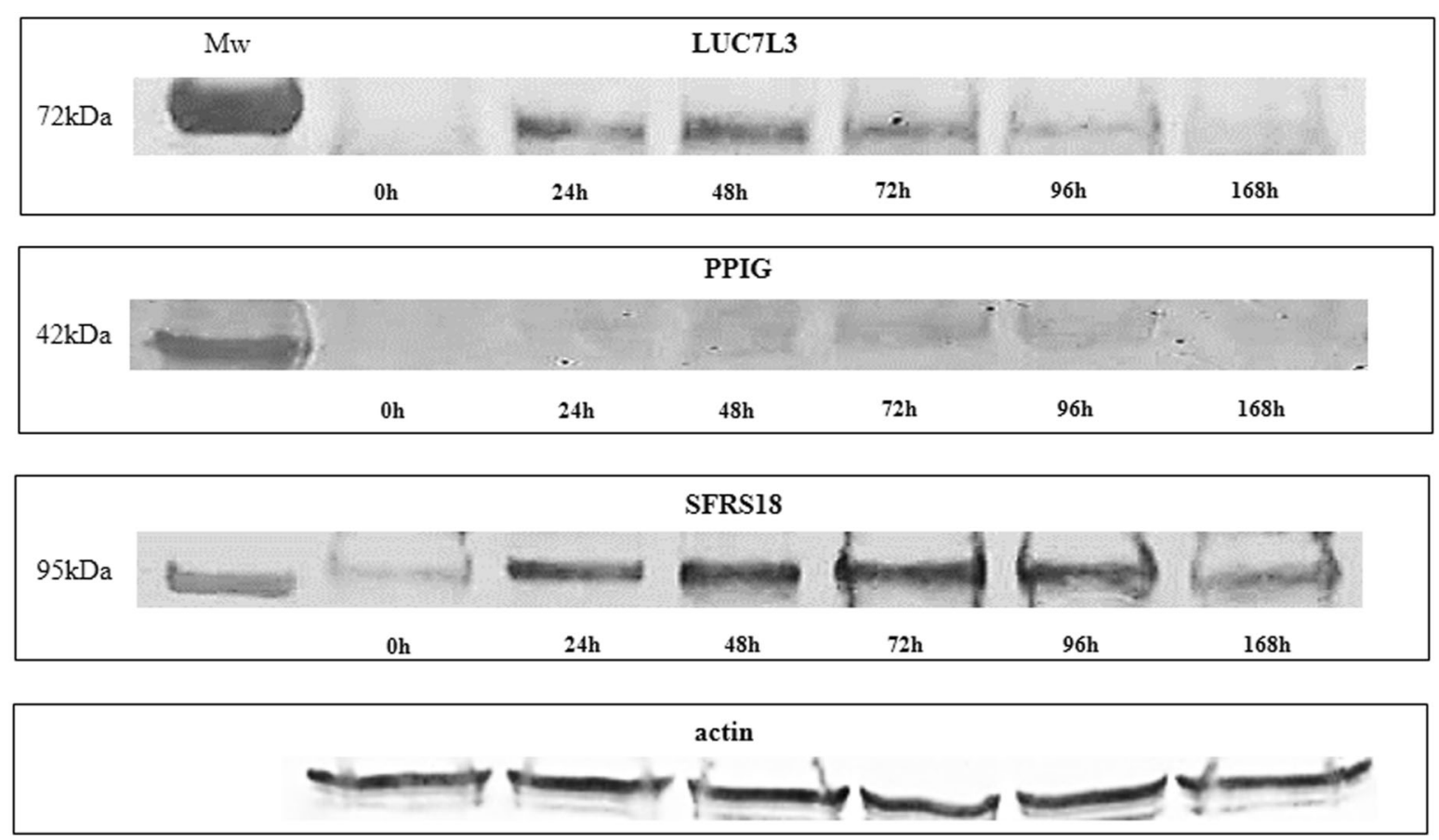

Fig. 3 HPV-KER cells were synchronized by contact inhibition and by withdrawal of supplement, and samples were taken at the indicated time points from the re-passaged $(0 \mathrm{~h})$ cells. Changes in mRNA levels (a) were followed by real-time RT-PCR and changes in protein levels (b) were followed by western blot analysis. The experiment was

\section{Silencing of PPIG, SFRS18, and $L U C 7 L 3$ affects fibronectin splicing}

To determine the physiological relevance of LUC7L3, PPIG, and SFRS18 in the fibronectin mRNA maturation processes, HPV-KER cells were transfected with siRNA constructs silencing these splicing-factor genes. The $\mathrm{EDA}+/$ total fibronectin ratios were compared in scrambled and gene-specific siRNA-transfected cells, and the mean of silencing efficacies was approximately $80 \%$ (summarized in Suppl. Fig. 2). Changes in the fibronectin splicing pattern were investigated by RT-PCR, and densitometry analysis was applied as an accurate and semiquantitative method to determine changes in the EDA+/total ratio in carried out with three independent series of cell cultures. The mRNA expression data were calculated as the average of these three parallel experiments. One representative western blot is provided for each splicing factor. $\alpha$-actin was used as a loading control

response to silencing of the three different splicing factors either alone or in combination.

In the experiments where a single splicing factor was silenced, siRNA transfection of LUC7L3 has lowered the EDA+/total ratio for fibronectin, and a slight decrease in this ratio was also seen with PPIG and SFRS18 silencing (Fig. 4a). In the scrambled RNA-transfected samples, the relative amount of the EDA+ variant was higher compared to the EDA - fibronectin isoform.

Bioinformatics analysis suggests that LUC7L3 and SFRS18 are interacting partners (Fig. 4c). Therefore, experiments in which two or three genes were silenced were also performed to examine the possible interactions among the splicing factors. The combined silencing of 


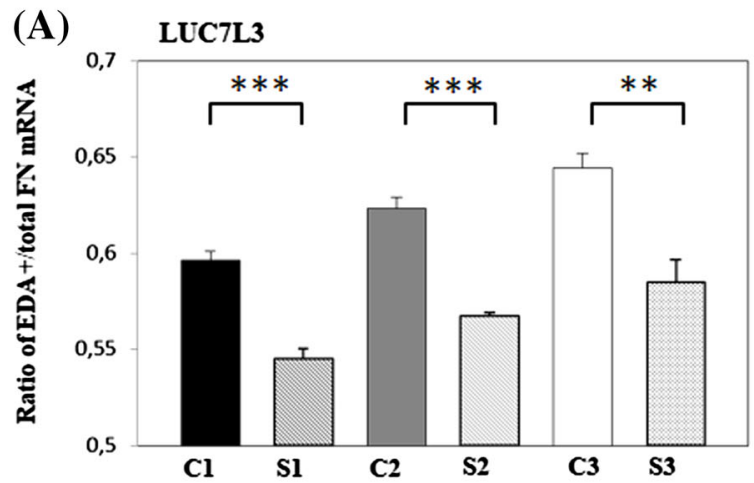

PPIG
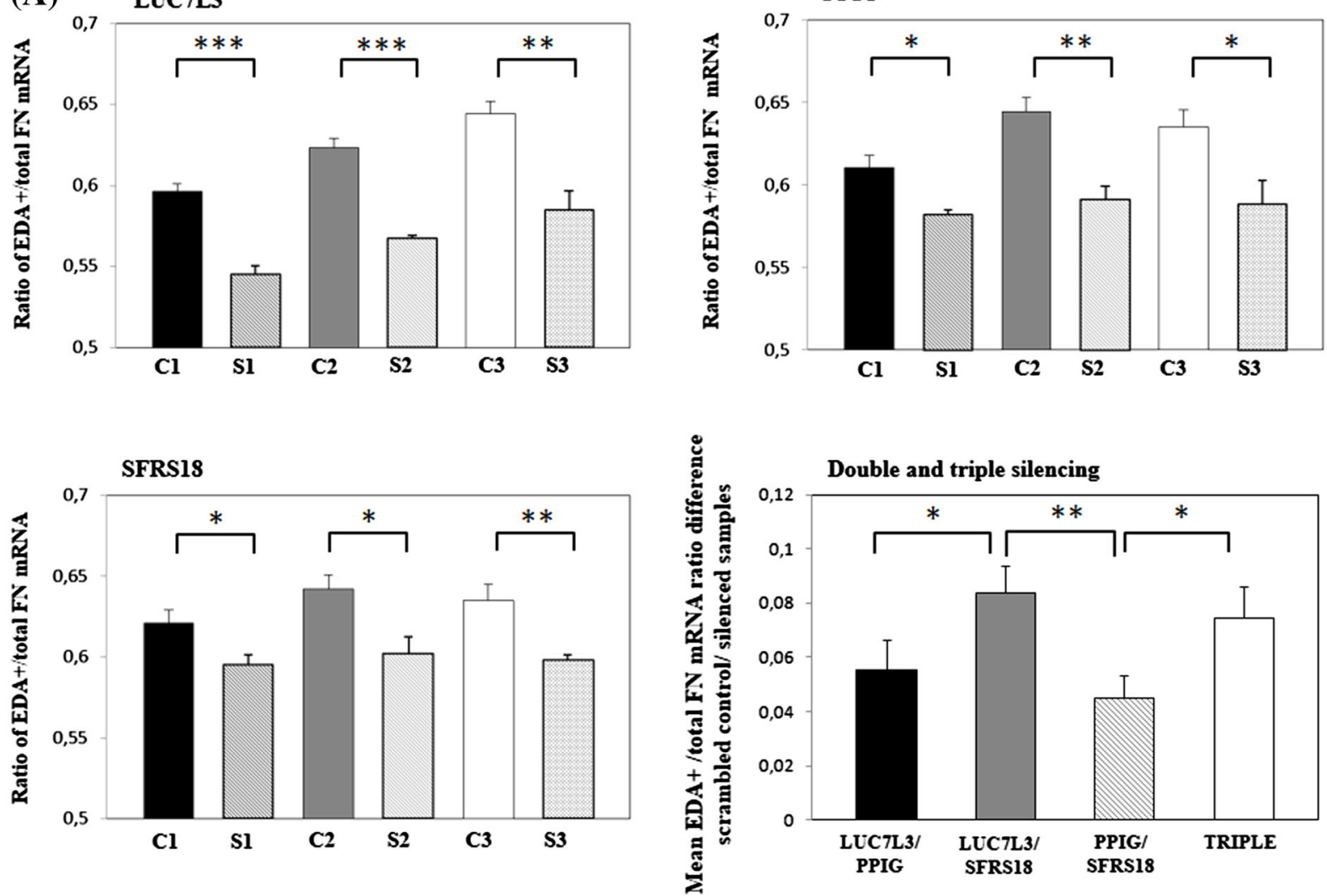

(B)

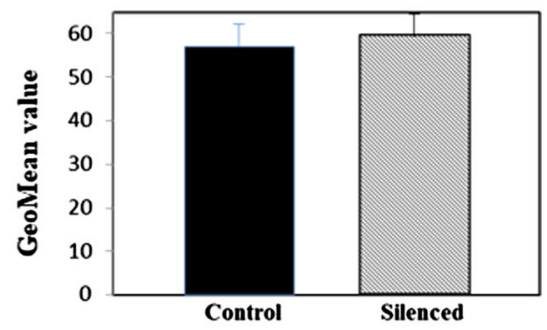

EDA+ FN

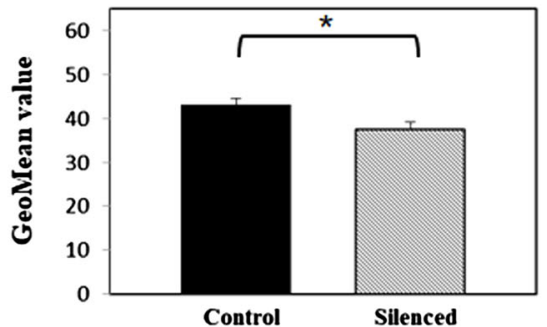

Control

Silenced

EDA +/total FN

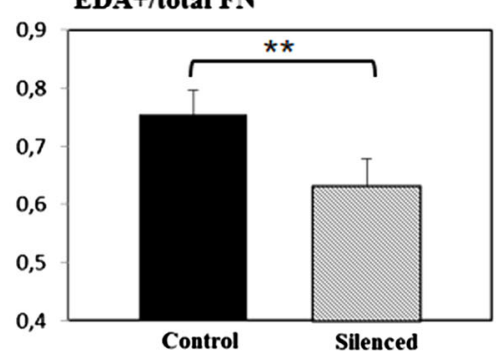

(C)

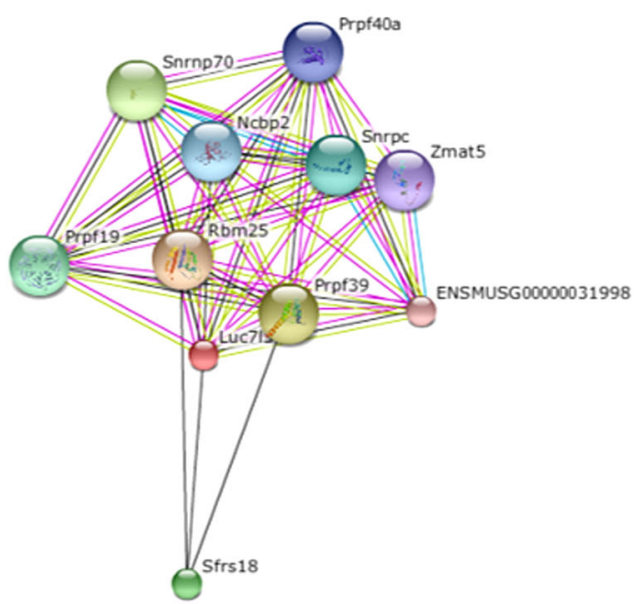

Rbm25

Prpf39

Snrnp70

Sfrs18

Prpf19

Snrpc

Ncbp2

Prpf40a

Zmat5

ENSMUSG00000031998

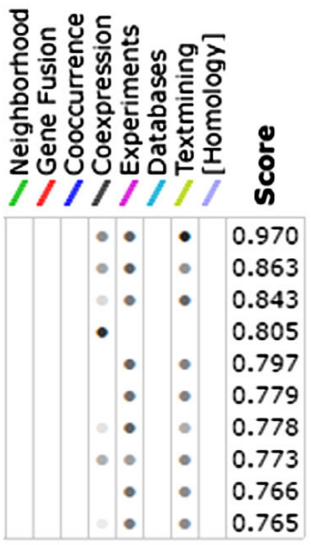


4Fig. 4 HPV-KER cells were transfected with specific silencing constructs for $L U C 7 L 3, P P I G$, and SFRS18 (a) and the ratio of $\mathrm{EDA}+$ fibronectin mRNA was calculated in each repeated experiment (S1, S2, S3). The changes in this ratio were compared to that of the scrambled RNA-transfected controls (C1, C2, C3) in each experiment. Comparison of the effect of double and triple silencing of the LUC7L3, PPIG, and SFRS18 genes to the scrambled RNAtransfected controls revealed that the double silencing of $L U C 7 L 3$ and SFRS18 resulted in the most robust changes in the EDA+/total ratio of fibronectin mRNA. Effect of triple silencing did not differ significantly from that of $L U C 7 L 3$ and SFRS18 double silencing. Columns represent means of three independent experiments $(+\mathrm{SE})$, all performed in triplicate. Significance levels are indicated by asterisks $(* p<0.05, * * p<0.01, * * * p<0.001)$. Total fibronectin and EDA+ fibronectin were detected by flow cytometry in HPV-KER cells $(n=5)$ in which both $L U C 7 L 3$ and SFRS18 were silenced (b). Silencing did not affect the amount of total fibronectin, whereas a significant $(p<0.05)$ decrease in the amount of the EDA+ fibronectin was detected after silencing, and this decrease resulted in significant $(p<0.01)$ changes in the EDA+/total ratio of fibronectin. Bioinformatics analysis revealed a predicted functional interaction between the LUC7L3 and SFRS18 splicing regulators (c)

LUC7L3 and SFRS18 leads to the most robust change in the EDA+/total ratio of fibronectin (Fig. 4a). Notably, silencing of all three splicing factors had no additional effect on the ratio compared to the double silencing of LUC7L3 and SFRS18.

The effect of silencing was also examined at the protein level. Total and EDA+ fibronectin amount was measured with flow cytometry using the most effective combination of double LUC7L3 and SFRS18 silencing. Similar to the mRNA experiments, the immortalized HPV-keratinocytes expressed high levels of EDA+ fibronectin protein. This double silencing significantly diminished the quantity of $\mathrm{EDA}+$ fibronectin, without decreasing the total amount of fibronectin (Fig. 4b). Taken together, these results demonstrate that silencing of the splicing factors decreased the EDA+/total fibronectin ratio both at mRNA and protein level.

\section{Discussion}

The importance of fibronectin as an excellent model for studying splicing events as well as the importance of various fibronectin isoforms-including variants containing the EDA and EDB domains-is increasingly being acknowledged. It was previously shown that EDA+ fibronectin is overexpressed in samples of psoriatic non-involved epidermis [5]. Some years later, our research group reported that both normal cultured keratinocytes and $\mathrm{HaCaT}$ cells produce the oncofetal EDA+ fibronectin isoform. Moreover, it has also been proven that keratinocytes from psoriatic non-involved epidermis are sources of EDA+ fibronectin, while the expression of this isoform is minimal in healthy keratinocytes. These data suggest that EDA+ fibronectin might initiate a signal transduction process that sensitizes keratinocytes to the mitogenic signals of immune cells [4].

In our recently performed cDNA microarray experiment, we aimed to reveal that novel molecular factors make it prone to form psoriatic lesions. From this work, we identified three splicing factors that participate in the molecular processes of mRNA maturation. In addition to regulation of splicing, PPIG is responsible for the normal folding of proteins, and LUC7L3 has been implicated in cisplatin resistance in tumor cells [27-29]. PPIG and SFRS18 are physical interactors of the splicing factor and the desmosome-associated protein, pinin [27, 30].

Our study opens the questions whether LUC7L3, PPIG, and SFRS18 are involved in the mRNA maturation process of fibronectin and whether the altered expression of these splicing factors can be linked to the documented presence of EDA+ fibronectin in psoriasis. Although EDA+ fibronectin is known to be involved in several biological processes, the regulation of its synthesis is not completely understood. TGF- $\beta$ is known to promote inclusion of EDA domain [31-33]. Other research groups reported that the SR-rich protein SF2/ASF exerts a similar effect, whereas heterogeneous ribonucleoprotein particles inhibit its inclusion $[8,34,35]$. According to the latest findings, keratinocyte growth factor (KGF) slightly increases the formation of the EDA+ variant, and STAT1 induces exclusion in fibroblasts [36].

Based on our results, LUC7L3 might have a direct regulatory role on EDA+ domain inclusion: similarly to the EDA+ fibronectin, its protein level is also increased in non-involved epidermis. SFRS18 showed only a minimal tendency towards elevation. PPIG amounts are decreased in non-involved epidermis compared to healthy controls, and this expression pattern resembles that which we previously observed for STAT1, another component of the EDA+ fibronectin-related molecular pathway. Moreover, similar to STAT1, PPIG proved to be part of the functional molecular network involving KGF and fibronectin signaling [36].

Another relevant outcome of our current study is that all three splicing factors exhibit the highest level of staining in psoriatic involved epidermis, with approximately 2- to 3 -fold elevation in involved epidermis compared to noninvolved samples. These data indicate that the examined splicing factors could influence the mRNA maturation of several other molecular components involved in the inflammatory and proliferative processes of psoriatic lesions.

In addition to tissue staining, results from in vitro characterization of splicing regulator expression also support the congruent pattern detected in psoriatic involved 
epidermis. In synchronized, immortalized keratinocytes, remarkable similarities in the expression patterns were observed both at the RNA and protein levels. Genetic linkage does not account for these similarities, as the LUC7L3, SFRS18, and PPIG genes are located on different chromosomes. Instead, the corresponding mRNAs might possess common transcription-factor binding sites and upstream regulators. Based on western blot analysis and immunofluorescent staining results, we conclude that expression of the three splicing factors is marked in both immortalized keratinocytes and involved epidermis. This finding supports that elevated amounts of LUC7L3, PPIG, and SFRS18 might be responsible for the shortened and abnormal maturation of keratinocytes that is a characteristic of psoriatic epidermis and is also typical of the immortalized HPV-KER cells.

The influence of the LUC7L3 splicing regulator on the formation of EDA+ fibronectin was also confirmed by in vitro experiments. We demonstrated that immortalized HPV-KER cells express the oncofetal EDA+ isoform of fibronectin in higher amounts than the EDA- isoform. LUC7L3 silencing resulted in the decrease of the $\mathrm{EDA}+/$ total ratio for fibronectin, indicating that the relative amount of EDA - fibronectin is elevated in response to LUC7L3 silencing. Similar results were obtained with silencing of PPIG and SFRS18, although the changes in the EDA+/total fibronectin ratios were more moderate than with $L U C 7 L 3$ silencing. These data suggest that the three splicing factors promote exon inclusion of the EDA domain. This finding is in accordance with previous work showing that another type of SR protein, SF2/ASF, supports the inclusion of the EDA element [34, 35].

Double silencing of SFRS18 and LUC7L3 was the most effective in decreasing the EDA+/total ratio of fibronectin, as expected from the results of bioinformatics analysis. Moreover, LUC7L3 and SFRS18 exhibited a similar pattern of expression during immunofluorescent staining. To analyze whether the changes in the EDA+/total fibronectin ratio detected by PCR are similar at the protein level, flow cytometric measurements were performed in cells in which both SFRS 18 and $L U C 7 L 3$ were silenced. The EDA+/total ratio for fibronectin protein decreased in these experiments. The quantity of total fibronectin was constant before and after silencing, and the ratio changes were thus due to increased production of the EDA - fibronectin isoform via an altered splicing mechanism. In addition to SF2/ASF, other SR-rich proteins have also been shown to promote inclusion of the EDA domain. This finding is of special interest, considering that SR proteins usually interact with enhancer splicing elements, inducing exon inclusion [37-39].

Several previous studies indicate that alternative splicing changes the binding characteristics of fibronectin radically $[6,8,13,40]$. The $\alpha 5 \beta 1$ integrin receptor is one of the most important interacting partners for fibronectin, and the inclusion of the EDA domain has been shown to increase affinity towards this receptor [6, 40, 41]. However, a contradicting report demonstrated that the EDA domain does not bind to $\alpha 5 \beta 1$ integrin [8]. It is also well documented that EDA+ fibronectin binds to the TLR4 receptor, which is part of the innate immune system [6, 33, 42]. It would be a very interesting new approach to study the changes of TLR4-mediated signal transduction in response to the altered expression of splicing factors.

Taken together, LUC7L3 might- through inclusion of the EDA domain and, thus, elevation of EDA+ fibronectin abundance in the epidermis — contribute to an autocrine loop by which psoriatic keratinocytes maintain hyper-responsiveness towards proliferative signals. Although the amounts of SFRS18 did not show elevation in the non-involved epidermis, in vitro studies support the suggestion that the presence of this protein could facilitate the effect of LUC7L3. All three splicing regulators are notably elevated in psoriatic involved epidermis, indicating that the mRNA maturation of fibronectin is influenced by these proteins, and that these regulators could also have multiple roles in disease development.

In future work, it would be interesting to clarify the upstream regulatory elements maintaining the highly synchronous expression of these three splicing factors and whether these elements are altered in psoriasis. Moreover, identification of additional mRNAs with altered expression and/or splicing patterns under the regulation of the LUC7L3, PPIG, and SFRS18 splicing factors might lead to the identification of novel molecular patterns in psoriasis pathogenesis.

Acknowledgements The study was supported by OTKA K105985, OTKA K111885 and TÁMOP-4.2.2.A-11/1/KONV-2012-0035, GINOP-2.3.2-15-2016-00015 research Grants.

\section{References}

1. Nestle FO, Kaplan DH, Barker J (2009) Psoriasis. N Engl J Med 361:496-509

2. Roberson D, Bowcock AM (2010) Psoriasis genetics: breaking the barrier. Trends Genet 26:415-423

3. Lowes MA, Suarez-Farinas M, Krueger JG (2014) Immunology of psoriasis. Annu Rev Immunol 32:227-255

4. Szell M, Bata-Csorgo Z, Koreck A, Pivarcsi A, Polyanka H, Szeg C, Gaal M, Dobozy A, Kemeny L (2004) Proliferating keratinocytes are putative sources of the psoriasis susceptibility-related EDA+ (extra domain A of fibronectin) oncofetal fibronectin. J Invest Dermatol 123:537-546

5. Ting KM, Rothaupt D, McCormick TS, Hammerberg C, Chen G, Gilliam AC, Stevens S, Culp L, Cooper KD (2000) Overexpression of the oncofetal Fn variant containing the EDA splice-in segment in the dermal-epidermal junction of psoriatic uninvolved skin. J Invest Dermatol 114:706-711

6. McFadden JP, Basketter DA, Dearman RJ, Kimber IR (2011) Extra domain A-positive fibronectin-positive feedback loops and 
their association with cutaneous inflammatory disease. Clin Dermatol 29:257-265

7. Kornblihtt AR, Pesce CG, Alonso CR, Cramer P, Srebrow A, Werbajh S, Muro AF (1996) The fibronectin gene as a model for splicing and transcription studies. FASEB J 10:248-257

8. White ES, Baralle FE, Muro AF (2008) New insights into form and function of fibronectin splice variants. J Pathol 216:1-14

9. Ffrench-Constant C, Van de Water L, Dvorak HF, Hynes RO (1989) Reappearance of an embryonic pattern of fibronectin splicing during wound healing in the adult rat. J Cell Biol 109:903-914

10. Lohi J, Tani T, Laitinen L, Kangas L, Lehto VP, Virtanen I (1995) Tenascin and fibronectin isoforms in human renal cell carcinomas, renal cell carcinoma cell lines and xenografts in nude mice. Int J Cancer 63:442-449

11. Astrof S, Crowley D, George EL, Fukuda T, Sekiguchi K, Hanahan D, Hynes RO (2004) Direct test of potential roles of EIIIA and EIIIB alternatively spliced segments of fibronectin in physiological and tumor angiogenesis. Mol Cell Biol 24:8662-8670

12. White ES, Muro AF (2011) Fibronectin splice variants: understanding their multiple roles in health and disease using engineered mouse models. IUBMB Life 63:538-546

13. Xu J, Mosher D (2011) The extracellular matrix: an overview. Biology of extracellular matrix. Springer, Heidelberg, pp 41-76

14. Baechler EC, Batliwalla FM, Reed AM, Peterson EJ, Gaffney PM, Moser KL, Gregersen PK, Behrens TW (2006) Gene expression profiling in human autoimmunity. Immunol Rev 210:120-137

15. Romanowska M, Yacoub N, Seidel H, Donandt S, Gerken H, Phillip S, Haritonova N, Artuc M, Schweiger S, Sterry W, Foerster J (2008) PPARdelta enhances keratinocyte proliferation in psoriasis and induces heparin-binding EGF-like growth factor. J Invest Dermatol 128:110-124

16. Gudjonsson JE, Ding J, Li X, Tejasvi T, Qin ZS, Ghosh D, Aphale A, Gumucio DL, Voorhees JJ, Abecasis GR, Elder JT (2009) Global gene expression analysis reveals evidence for decreased lipid biosynthesis and increased innate immunity in uninvolved psoriatic skin. J Invest Dermatol 129:2795-2804

17. Blumenberg M (2012) SKINOMICS: transcriptional profiling in dermatology and skin biology. Curr Genomics 13:363-368

18. Manczinger M, Kemeny L (2013) Novel factors in the pathogenesis of psoriasis and potential drug candidates are found with systems biology approach. PLoS ONE 8:e80751

19. Villasenor-Park J, Ortega-Loayza AG (2013) Microarray technique, analysis, and applications in dermatology. J Invest Dermatol 133:e7

20. Keermann M, Koks S, Reimann E, Prans E, Abram K, Kingo K (2015) Transcriptional landscape of psoriasis identifies the involvement of IL36 and IL36RN. BMC Genomics 16:322

21. Li B, Tsoi LC, Swindell WR, Gudjonsson JE, Tejasvi T, Johnston A, Ding J, Stuart PE, Xing X, Kochkodan JJ, Voorhees JJ, Kang HM, Nair RP, Abecasis GR, Elder JT (2014) Transcriptome analysis of psoriasis in a large case-control sample: RNA-seq provides insights into disease mechanisms. J Invest Dermatol 134:1828-1838

22. Koks S, Keermann M, Reimann E, Prans E, Abram K, Silm H, Koks G, Kingo K (2016) Psoriasis-specific RNA isoforms identified by RNA-seq analysis of 173,446 transcripts. Front Med (Lausanne) 3:46

23. Szabo K, Bata-Csorgo Z, Dallos A, Bebes A, Francziszti L, Dobozy A, Kemeny L, Szell M (2014) Regulatory networks contributing to psoriasis susceptibility. Acta Derm Venereol 94:380-385

24. Bata-Csorgo Z, Hammerberg C, Voorhees JJ, Cooper KD (1995) Kinetics and regulation of human keratinocyte stem cell growth in short-term primary ex vivo culture. Cooperative growth factors from psoriatic lesional $\mathrm{T}$ lymphocytes stimulate proliferation among psoriatic uninvolved, but not normal, stem keratinocytes. J Clin Invest 95:317-327

25. Polyanka H, Szabo K, Tax G, Tubak V, Kusz E, Ujfaludi Z, Boros I, Bata-Csorgo Z, Kemeny L, Szell M (2011) Primary characterization of a novel HPV- E6 oncogene immortalized keratinocyte cell line. J Invest Dermatol 131(Suppl. 2):S70

26. Pivarcsi A, Szell M, Kemeny L, Dobozy A, Bata-Csorgo Z (2001) Serum factors regulate the expression of the proliferationrelated genes alpha5 integrin and keratin 1, but not keratin 10, in HaCaT keratinocytes. Arch Dermatol Res 293:206-213

27. Lin CL, Leu S, Lu MC, Ouyang P (2004) Over-expression of SRcyclophilin, an interaction partner of nuclear pinin, releases SR family splicing factors from nuclear speckles. Biochem Biophys Res Commun 321:638-647

28. Nishii Y, Morishima M, Kakehi Y, Umehara K, Kioka N, Terano Y, Amachi T, Ueda K (2000) CROP/Luc7A, a novel serine/ arginine-rich nuclear protein, isolated from cisplatin-resistant cell line. FEBS Lett 465:153-156

29. Puig O, Bragado-Nilsson E, Koski T, Seraphin B (2007) The U1 snRNP-associated factor Luc7p affects $5^{\prime}$ splice site selection in yeast and human. Nucleic Acids Res 35:5874-5885

30. Zimowska G, Shi J, Munguba G, Jackson MR, Alpatov R, Simmons MN, Shi Y, Sugrue SP (2003) Pinin/DRS/memA interacts with SRp75, SRm300 and SRrp130 in corneal epithelial cells. Invest Ophthalmol Vis Sci 44:4715-4723

31. Balza E, Borsi L, Allemanni G, Zardi L (1988) Transforming growth factor beta regulates the levels of different fibronectin isoforms in normal human cultured fibroblasts. FEBS Lett 228:42-44

32. Borsi L, Castellani P, Risso AM, Leprini A, Zardi L (1990) Transforming growth factor-beta regulates the splicing pattern of fibronectin messenger RNA precursor. FEBS Lett 261:175-178

33. Kelsh RM, McKeown-Longo PJ, Clark RA (2015) EDA fibronectin in keloids create a vicious cycle of fibrotic tumor formation. J Invest Dermatol 135:1714-1718

34. Caputi M, Casari G, Guenzi S, Tagliabue R, Sidoli A, Melo CA, Baralle FE (1994) A novel bipartite splicing enhancer modulates the differential processing of the human fibronectin EDA exon. Nucleid Acids Res 22:1018-1022

35. Buratti E, Muro AF, Giombi Gherbassi D, Iaconcig A, Baralle FE (2004) RNA folding affects the recruitment of SR proteins by mouse and human polypurinic enhancer elements in the fibronectin EDA exon. Mol Cell Biol 24:1387-1400

36. Guban B, Vas K, Balog Z, Manczinger M, Bebes A, Groma G, Szell M, Kemeny L, Bata-Csorgo Z (2016) Abnormal regulation of fibronectin production by fibroblasts in psoriasis. Br J Dermatol 174:533-541

37. Faustino NA, Cooper TA (2003) Pre-mRNA splicing and human disease. Genes Dev 17:419-437

38. Pagani F, Baralle FE (2010) Analysis of human splicing defects using hybrid minigenes. Molecular diagnostics. Elsevier, New York, pp 155-169

39. Ward AJ, Cooper TA (2011) The pathobiology of splicing. J Pathol 220:152-163

40. Pankov R, Yamada KM (2002) Fibronectin at glance. J Cell Sci 115:3861-3863

41. Pellegrini G, De Luca M, Orecchia G, Balzac F, Cremona O, Savoia P, Cancedda R, Marchisio PC (1992) Expression, topography, and function of integrin receptors are severely altered in keratinocytes from involved and uninvolved psoriatic skin. J Clin Invest 89:1783-1795

42. Okamura KY, Watari M, Jerud ES, Young DW, Ishizaka ST, Rose J, Chow JC, Strauss JF (2002) The extra domain A of fibronectin activates Toll-like receptor 4. J Biol Chem 276:10229-10233 


\title{
Erratum to: Splicing factors differentially expressed in psoriasis alter mRNA maturation of disease-associated EDA+ fibronectin
}

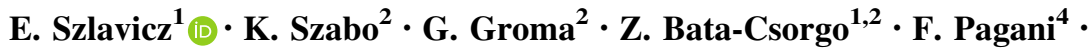 \\ L. Kemeny ${ }^{1,2} \cdot$ M. Szell ${ }^{2,3}$
}

(C) Springer Science+Business Media, LLC 2017

\section{Erratum to: Mol Cell Biochem}

DOI 10.1007/s11010-017-3090-1

The original article was published with error in an abstract. The word "facwwtors" in the seventh sentence should read as "factors".

The online version of the original article can be found under doi:10.1007/s11010-017-3090-1.

E. Szlavicz

szlavicz.eszter@gmail.com

1 Department of Dermatology and Allergology, Faculty of Medicine, University of Szeged, 6 Korányi fasor, Szeged 6720, Hungary

2 MTA-SZTE Dermatological Research Group, Szeged, Hungary

3 Department of Medical Genetics, Faculty of Medicine, University of Szeged, Szeged, Hungary

4 International Centre for Genetic Engineering and Biotechnology, Trieste, Italy 


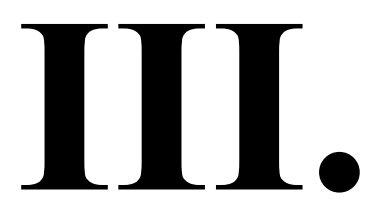




\title{
U $T$ World Journal of Dermatology
}

Online Submissions: http:/ / www.wjgnet.com/esps/ Help Desk: http:/ / www.wjgnet.com/esps/helpdesk.aspx doi:10.5314/wjd.v3.i3.50
World J Dermatol 2014 August 2; 3(3): 50-57

ISSN 2218-6190 (online)

(c) 2014 Baishideng Publishing Group Inc. All rights reserved.

\section{What have we learned about non-involved psoriatic skin from large-scale gene expression studies?}

\author{
Eszter Szlavicz, Kornelia Szabo, Zsuzsanna Bata-Csorgo, Lajos Kemeny, Marta Szell
}

Eszter Szlavicz, Zsuzsanna Bata-Csorgo, Lajos Kemeny, Department of Dermatology and Allergology, University of Szeged, H-6720 Szeged, Hungary

Kornelia Szabo, Zsuzsanna Bata-Csorgo, Lajos Kemeny, Marta Szell, Dermatological Research Group of the Hungarian Academy of Sciences, University of Szeged, H-6720 Szeged, Hungary

Marta Szell, Department of Medical Genetics, the University of Szeged, H-6720 Szeged, Hungary

Author contributions: Szlavicz E contributed to experimental work on the splice regulatory genes, review of previous papers, writing of the manuscript; Szabo K contributed to experimental work with the microarray-identified genes; Bata-Csorgo Zs contributed to co-ordination of the clinical aspects of the work, collection of skin specimen; Kemeny L contributed to co-ordination of the clinical and experimental aspects of psoriasis research; Szell M contributed to experimental work on the differentialdisplay-identified genes, supervising experimental aspects of the review.

Supported by OTKA NK77434, OTKA K 83277, OTKA K105985 and TÁMOP-4.2.2.A-11/1/KONV, TÁMOP-4.2.2-B-10/1-2010-0012; the Bolyai Foundation of the Hungarian Academy of Sciences (to Kornelia Szabo)

Correspondence to: Eszter Szlavicz, MD, Department of Dermatology and Allergology, University of Szeged, Koranyi fasor 6, H-6720 Szeged, Hungary. szlavicz.eszter@gmail.com Telephone: +36-62-545277 Fax: +36-62-545954

Received: December 29, 2013 Revised: May 22, 2014

Accepted: May 28, 2014

Published online: August 2, 2014

\section{Abstract}

Psoriasis is a chronic inflammatory skin disorder; its genetic background has been widely studied in recent decades. Recognition of novel factors contributing to the pathogenesis of this disorder was facilitated by potent molecular biology tools developed during the 1990s. Large-scale gene expression studies, including differential display and microarray, have been used in experimental dermatology to a great extent; moreover, skin was one of the first organs analyzed using these methods. We performed our first comprehensive gene expression analysis in 2000. With the help of differential display and microarray, we have discovered several novel factors contributing to the inherited susceptibility for psoriasis, including the EDA+ fibronectin splice variant and PRINS. The long non-coding PRINS RNA is expressed at higher levels in non-involved skin compared to healthy and involved psoriatic epidermis and might be a factor contributing cellular stress responses and, specifically, to the development of psoriatic symptoms. This review summarizes the most important results of our large-scale gene expression studies.

(c) 2014 Baishideng Publishing Group Inc. All rights reserved.

Key words: Non-involved psoriatic skin; Differential display; cDNA microarray; EDA+ fibronectin isoform; PRINS long non-coding RNA; mRNA maturation

Core tip: Large-scale gene expression studies, including differential display and microarray, have provided valuable data on the molecular background of psoriasis pathogenesis. This review summarizes the most important results of the available literature and our large-scale gene expression studies obtained from the clinically non-involved psoriatic skin: we identified the EDA+ fibronectin splice variant as an autocrine proliferation signal for psoriatic hyperproliferative keratinocytes and PRINS, a long non-coding regulatory RNA. We believe that the characterization of new candidate genes and proteins might establish new therapeutic approaches, which may allow treatment of already existing psoriatic lesions as well as non-involved psoriatic skin by affecting molecular aberrancies, and may lead to the development of prophylactic interventions.

Szlavicz E, Szabo K, Bata-Csorgo Zs, Kemeny L, Szell M. What have we learned about non-involved psoriatic skin from largescale expression studies? World J Dermatol 2014; 3(3): 50-57 Available from: URL: http://www.wjgnet.com/2218-6190/full/ v3/i3/50.htm DOI: http://dx.doi.org/10.5314/wjd.v3.i3.50 


\section{INTRODUCTION}

Psoriasis is a hyperproliferative inflammatory skin disorder affecting approximately $2 \%-3 \%$ of the European population $^{[1]}$. However, in some other parts of the world, this disease is almost unknown: e.g., in Africa the occurrence of psoriatic cases is remarkably rare ${ }^{[2]}$. The exact trigger of the disease is still obscured and the subject of several investigations. Inherited and environmental factors (e.g., mechanical trauma, UV exposure, stress) are responsible for the development of psoriatic symptoms ${ }^{[2,3]}$.

In the most typical cases, hyperproliferative psoriatic plaques are formed on the skin of the knees, elbows and the scalp. In addition, the disorder can affect skin annexes and joints. In the case of some patients with severe psoriasis, the entire body is covered with lesions. Severe psoriasis is often associated with metabolic syndrome; hence, psoriasis patients also have elevated cardiovascular and stroke risks ${ }^{[2]}$. Unfortunately, psoriasis has a negative effect on the patient's quality of life due to serious psychosocial and emotional stress ${ }^{[4]}$. A number of emerging arguments support the idea that psoriasis is a systemic disorder rather than simply a skin disease. Psoriasis has many common features with chronic autoimmune inflammatory disorders, such as progressive arthritis. Moreover, psoriasis and autoimmune syndromes often share common genetic loci ${ }^{[5-7]}$. Similarities are especially evident when psoriasis is compared to chronic inflammatory bowel disorders, such as Crohn's disease, where internal barriers are involved ${ }^{[8,9]}$.

Similarly to autoimmune disorders, immune-activation plays an important role in psoriasis: the development of the characteristic erythematosus, demarcated and scaly lesions is related to the abnormal functioning of the cellular immune system ${ }^{[10-14]}$. Cytokines produced by aberrantly functioning T-lymphocytes are able to stimulate keratinocytes, which show an elevated sensitivity to these proliferative signals ${ }^{[14,15]}$. However, it is still unknown whether the primary triggers of the disease phenotype are the professional immune cells or the keratinocytes. Inherited susceptibility of keratinocytes has been partially established. Identification and characterization of these factors may greatly facilitate the understanding of the molecular background of psoriasis. Large-scale gene expression profiling methods developed and used in the 1990s might be useful tools to answer these exciting questions.

\section{DAWN OF THE LARGE-SCALE GENE EXPRESSION STUDIES: DIFFERENTIAL DISPLAY AND SAGE}

In recent years, we and others have tried to characterize molecular factors responsible for the hyper responsiveness of keratinocytes to various stimuli ${ }^{[8,12,14,16]}$. To reveal these processes, researchers need suitable and powerful methods that can detect more than one possible target.
Previously, altered expression of only a few candidate genes or proteins was possible. The development of large-scale gene expression analysis methods marked a significant breakthrough in this field. With the help of microarrays and their predecessors, differential display (DD) and the serial analysis of gene expression (SAGE), gene-expression patterns of serial samples can be compared for large data sets.

For DD, gene expression profiles are analyzed for pairs of corresponding sample sets. The most important steps of the method are the isolation of total RNA from the samples and its reverse transcription into cDNA. Subsequently, cDNA is amplified, subjected to gel electrophoresis and, after the expression pattern has been compared, bands representing differentially expressed genes are cut out from the gel and the DNA content is cloned into a plasmid vector. It should be mentioned that the DD method has some limitations due to the relatively frequent incidence of false positive results. Hence, the results must be validated using an independent technique. Validation is usually carried out by reverse Southern blot analysis, followed by sequencing the differentially expressed transcripts ${ }^{[17-19]}$. The great advantage of DD is that it is an "open ended" analysis system, allowing unannotated differentially expressed transcripts to be identified.

Another sequence-based approach, SAGE, was developed at the Oncology Center of the Johns Hopkins University by Velculescu and his co-workers. Changes in gene expression patterns are detected by sequencing reverse transcribed cDNAs. Application of short oligonucleotide sequence tags allows quantitative changes to be monitored, in addition to the qualitative analysis ${ }^{[0,21]}$.

\section{MICROARRAYS}

Microarrays provide more extended and comprehensive methods for analyzing gene expression profiles than DD and SAGE. The biggest advantage of this approach is that it allows thousands of genes to be measured simultaneously. Moreover, complex regulatory networks can be assessed $^{[22,23]}$. In contrast to DD and SAGE, microarrays are a "closed" analysis system, allowing only known sequences to be screened. The introduction and widespread use of microarrays has facilitated advances in several branches of science, including experimental dermatology. In fact, the skin was one of the first human organs to be analyzed with this technique ${ }^{[24-27]}$.

Microarray technologies rely on complementarity for sequence-specific recognition of the DNA segments ${ }^{[28]}$. Most commonly used probes are cDNAs derived from bacterial libraries and BACs or oligonucleotides. Long oligonucleotide probes (50-120 nt) might support a higher degree of specificity and sensitivity than short (15-25 nt) probes $^{[22]}$. The probes are fixed to a solid support, such as glass or plastic that are referred to as "chips" in common laboratory jargon ${ }^{[23]}$.

For microarray experiments, total RNA is isolated 
from samples and reverse-transcribed with fluorescent dyes such as $\mathrm{Cy} 3$ and $\mathrm{Cy} 5$ or with radioactive isotope to label the synthesized cDNA. After hybridizing the labeled probes to the chips for approximately 16-24 h, the chips are washed and the fluorescence is scanned with a confocal microscopy. Data are then analyzed using specially developed software. Like DD, this method can identify false positives and, therefore, must be validated by RT-PCR, northern blot analysis or RNAse protection assay ${ }^{[22,28]}$.

The outstanding advantage of microarray techniques is the simultaneous investigation of thousands of genes and, thus, the possibility to explore novel molecular pathways. This technique can be a powerful tool in tumor and biomarker research and may serve as the basis of personalized therapies ${ }^{[27]}$.

\section{LARGE-SCALE GENE EXPRESSION STUDIES OF PSORIASIS: IDENTIFICATION OF MOLECULAR FACTORS CONTRIBUTING TO PATHOGENESIS}

The use of large-scale gene-expression analysis methods has been fruitful for experimental dermatology. Microarrays have been used to study several disorders, such as melanoma, atopic dermatitis and autoimmune skin diseases. In the past decade, DD and microarray techniques have been widely employed alone or in combination with other methods in psoriasis research ${ }^{[29]}$.

Gene-expression profiling of peripheral blood cells and epidermis samples from healthy, psoriatic involved and psoriatic non-involved skin proved to be a powerful tool for the characterization of aberrant molecular patterns in the disease ${ }^{[30]}$. The results of cDNA microarrays supported previous findings and were useful to describe novel pathways implicated in psoriasis pathogenesis. Psoriasis research was dominated by the so-called "immune theory" for many years, and microarray studies further proved the involvement of genes related to inflammation and immune responses. One of the earliest microarrays identified several inflammation- and immune-related genes (IL4R, CD2, CD24 and INF- $\gamma$ induced genes) that were not previously reported to contribute to the pathogenesis of this disorder ${ }^{[30,31]}$. Moreover, Oestreicher et $a l^{[31]}$ performed a longitudinal analysis in which they characterized changes in gene expression in response to recombinant human IL-1 or cyclosporine in therapy responder and non-responder populations. A study from Zhou, which compared samples from healthy, involved psoriatic and non-involved psoriatic skin biopsies further supported the involvement of the activated T-cell product INF- $\gamma$ and transcription factors induced by this proinflammatory lymphokine ${ }^{[30,32]}$. The role of IL-17 signaling was also demonstrated in large-scale gene-expression studies ${ }^{[13]}$. In addition, Gudjonsson et al ${ }^{[3]}$ emphasized the role of altered innate immune functions in psoriasis. Dif- ferential expression of genes encoding chemokines and their receptors were also described by several research groups $^{[32-34]}$.

Other important cellular pathways related to psoriasis regulate epidermal keratinocyte proliferation and apoptosis. The implication of PPAR- $\delta, m T O R, N F_{\kappa} B$, $B C L-2$ and $B A X$ expression was verified for these mechanisms ${ }^{[35-37]}$. In a study of Wnt pathways responsible for stem cell proliferation and differentiation, Reischl et al ${ }^{[38]}$ found that only $W n+5 a$ expression was higher in psoriatic involved skin than in non-involved samples. In addition, actin cytoskeleton organization can be affected: the $C C N A 2$ gene is responsible for the $\mathrm{G}_{2} / \mathrm{M}$ transition in the cell cycle and affects intracellular cytoskeleton organization and cell migration ${ }^{[39,40]}$.

The clinical association of psoriasis and metabolic syndrome is a well-known phenomenon. Gudjonsson and co-workers were able to show that lipid metabolism pathways were altered in psoriatic non-involved epidermis compared to healthy samples ${ }^{[3]}$. In this comparison, it was proven that lipid metabolism genes were downregulated in non-involved skin samples as compared to healthy skin and further down-regulation was identified in psoriatic involved $\operatorname{skin}^{[3]}$. Romanowska et al ${ }^{[35]}$ studied the role of PPAR $\delta$, a transcription factor participating in metabolic and inflammatory processes, in psoriasis. PPAR $\delta$ exerts proangiogenic and antiapoptotic affects and is suspected to be involved in the enhancement of keratinocyte proliferation ${ }^{[35]}$.

Most recently, bioinformatic meta-analyses were performed using publicly available databases of psoriasisrelated microarray data. In one of the first microarray meta-analysis, Tian $e t a l^{[41]}$ analyzed the result of five previous cDNA microarrays experiments. In a subsequent meta-analysis, Manczinger et al ${ }^{[40]}$ compared differentially expressed genes of psoriatic involved and non-involved epidermis. The findings of these two meta-analyses agreed and showed that the most important components of the molecular networks related to psoriasis are factors implicated in cell proliferation and immunomodulation. Importantly, these meta-analyses confirmed that several differentially expressed transcripts were also involved in metabolic disturbances, such as impaired glucose tolerance, insulin tolerance and atherosclerosis ${ }^{[40,41]}$.

It is important to note that most of the large-scale gene expression studies for the identification of molecular patterns in psoriasis pathogenesis have compared the gene expression profiles of psoriatic involved and non-involved skin or psoriatic involved and healthy skin. This research provided extremely valuable data for the molecular events of psoriasis ${ }^{[40]}$. Much less information is available, however, on differentially expressed genes in normal epidermis compared to psoriatic non-involved epidermis. We and others believe that identifying aberrantly expressed genes and molecular patterns in noninvolved psoriatic epidermis is important for understanding this disease. 


\section{DIFFERENTIAL DISPLAY AND MICROARRAY EXPERIMENTS OF OUR RESEARCH GROUP, FOR THE IDENTIFICATION OF NOVEL MOLECULAR FACTORS OF PSORIASIS}

Our research group performed the first comprehensive gene-expression analysis for psoriasis in 2000 to compare psoriatic non-involved epidermal samples with control healthy epidermis. This approach allowed early and inherited molecular factors to be studied in detail and allowed novel susceptibility factors to be revealed. This study identified two known transcripts that were differentially expressed: $R A B 10$, an oncogene that belongs to the small GTPase superfamily, and fibronectin, a well-known extracellular matrix component ${ }^{[42]}$. Our subsequent studies focused on the role of fibronectin in the pathogenesis of psoriasis.

Fibronectin is a complex glycoprotein composed of repetitive modules ${ }^{[43]}$. At least 24 differentially spliced variants of this gene have been described, and the presence of certain variants depends on age, developmental state and cell type ${ }^{[44]}$. Alternative processing involves three preferred sites: extra domain A (EDA), extra domain $\mathrm{B}$ and extra type homology $\mathrm{B}^{[43,45]}$. The splice variants containing the EDA domain play a crucial role in embryonic development and wound healing. However they are detectable only in modest amounts in adult normal tissues $^{[44,46,47]}$. Because it is also abundantly expressed in different types of tumors, it is referred to as the oncofetal fibronectin splice variant ${ }^{[48]}$. Interestingly, in the brain, an organ in which fibronectin is poorly expressed, the inclusion of the EDA domain is abundant in young adults (88\% as compared to fetal level) and decreases with age to $33 \%{ }^{[46]}$.

The presence of the EDA+ fibronectin variant is associated with several pathological conditions and is suspected to participate in the development of psoriasis as well. The oncofetal fibronectin form was found to be present in a higher ratio at the dermal-epidermal junction of psoriatic non-involved skin compared to healthy normal skin ${ }^{[14,49]}$. Unlike the conventional variant, the oncofetal EDA+ fibronectin form interacts with the $\alpha 5$ integrin subtype, instead of $\alpha 2$ and $\alpha 3$, and, as a result, its effect on cellular signaling processes is more robust. $\alpha 5 \beta 1$ integrin receptors were shown to be upregulated in both non-involved and involved psoriatic skin ${ }^{[14,50]}$.

In addition, other authors reported that the EDA+ fibronectin variant is co-localized with CD11c+ macrophages. It was suggested that these cells might contribute to the production of the oncofetal variant; however, because of their relatively low number, they are likely not to be the most important source ${ }^{[49]}$. Based on our results we supposed that keratinocytes themselves might produce EDA+ fibronectin and, as an autocrine molecular factor, may contribute to the induction and maintenance of ke- ratinocyte hyperproliferation in psoriasis ${ }^{[16]}$.

We have also performed in vitro experiments to understand the role of $\mathrm{EDA}+$ fibronectin in the regulation of keratinocyte proliferation. Subsequently, RT-PCR was carried out using immortalized $\mathrm{HaCa} \mathrm{T}$ cells. Our results indicated that, after serum starvation and contact inhibition, the highest level of EDA+ fibronectin expression could be detected in the highly proliferative $\mathrm{HaCaT}$ cells, and the ratio of EDA+/EDA- fibronectin produced by the keratinocytes might well be a potent mitogen signal in cell cycle regulation. In contrast to fibroblasts and normal human keratinocytes, the ratio was altered in this cell line. Flow cytometry supported the RT-PCR results. The results of the $\mathrm{HaCaT}$ cell line experiments indicated that keratinocytes themselves might produce the oncofetal fibronectin variant ${ }^{[16]}$.

In addition to proteins with known functions, the DD experiment identified a novel transcript: the corresponding gene was subsequently named psoriasis-susceptibilityrelated RNA gene induced by stress (PRINS, accession number AK022043). During the structural investigation of PRINS, we found that the gene consists of two exons containing several stop codons, which prevent the formation of a longer open reading frame. In silico sequence comparison supported the hypothesis that PRINS functions as a non-coding RNA molecule, rather than serving as a template for protein translation. In addition, PRINS contains two repetitive $A l u$ sequences and has $70 \%$ sequence similarity with the Tetrabymena thermophyla G8 small nucleolar non-coding $\mathrm{RNA}^{[42]}$.

In a quantitative RT-PCR analysis, we demonstrated that PRINS is expressed at higher levels in non-involved skin compared to healthy and involved psoriatic epidermis. Our in vitro experiments performed on synchronized HaCaT cells showed that PRINS expression dropped significantly when the cells were released from cell quiescence and the cells started to proliferate actively ${ }^{[42]}$. These data suggested that PRINS might be a factor disposing keratinocytes to hyperproliferation and contributing to the development of psoriatic symptoms. The exact role of PRINS is still unknown, but it is very possible that it plays an important role in cellular stress responses. Silencing PRINS did not affect the survival of the cells; however under certain stress conditions (such as serum starvation) the cells died at a much higher rate when the expression of PRINS was down-regulated ${ }^{[42,51]}$. Consequently, the PRINS-silenced cells became more vulnerable, supporting the cellular-stress response hypothesis. Moreover, our research group later showed that the G1P3 antiapoptotic protein might be regulated by the PRINS non-coding $\mathrm{RNA}^{[52]}$.

Since then, we have identified nucleophosmin as one of the possible cellular interacting partners of PRINS. Nucleophosmin is a phosphoprotein which is a member of the p53 pathway, and its movement in fibroblasts, cancer cells and keratinocytes is triggered by ultraviolet (UV) exposure $^{[53]}$. We also demonstrated that silencing PRINS prevents nucleolar-cytoplasmic shuttling of nucleophosmin. 
This result indicates that PRINS might physically interact with the nucleophosmin protein and that the abnormal functioning of the PRINS-nucleophosmin ribonucleoprotein complex may contribute to psoriasis pathogenesis ${ }^{[54]}$.

Taken together, we consider the identification of novel factors implicated in the early molecular defects in psoriasis pathogenesis-the EDA+ fibronectin splice-variant and the PRINS non-coding RNA-the most significant outcomes of our DD experiments. Due to the success of the DD, we attempted to identify novel psoriasis susceptibility factors using newly available cDNA microarray technology for large-scale gene-expression analysis. In particular, we aimed to identify molecular patterns that are responsible for the differential reactivity of normal healthy epidermis and psoriatic non-involved epidermis.

Organotypic tissue cultures were created from four healthy and four psoriatic non-involved skin samples. Half of the samples were treated with a mixture of T-cell lymphokines, containing IL-3, IFN $\gamma$ and GM-CSF, cytokines previously described to be implicated in the T-cell response and the formation of psoriatic plaques ${ }^{[10]}$. After three days of treatment, the dermis and epidermis were separated. Total RNA was isolated from the epidermis, reverse transcribed and used to perform the cDNA microarray experiment. Based on the results, we selected genes that showed an altered gene expression in response to the lymphokine treatment ${ }^{[12]}$.

We identified 61 transcripts that exhibited altered gene expression. Of these, eleven had been demonstrated earlier to contribute to psoriasis. Using bioinformatics tools, such as Gene Ontology and Ingenuity pathway analysis, we demonstrated that most of these molecules are implicated in two important intracellular pathways: "apoptosis" and "metabolism of small molecules and lipids." Realtime RT-PCR validation experiments revealed that many of these genes are already upregulated in non-involved psoriatic epidermis, and the lymphokine treatment did not further increase expression. In contrast, expression of these genes was inducible in healthy samples. These data indicate that keratinocytes in psoriatic non-involved epidermis are in a presensitised status, which explains their altered response to different triggering stimuli ${ }^{[12]}$.

Among the differentially expressed genes, we also identified members of the serine-arginine rich (SR) proteins SR splicing factor 18 (SFRS18), peptidylprolyl isomerase G (PPIG) and luc-7 like 3 (LUC7L3), which regulate mRNA splicing. It was previously described that these proteins interact with pinin and SR-related nuclear protein $^{[55-60]}$. Splicing is a post-transcriptional regulatory process and one of the most important sources of mRNA diversity, permitting the production of different mRNAs from the same DNA template. Splicing dysfunction has been shown to be involved in several disorders, and some novel therapeutic modalities have been designed to repair them ${ }^{[61-63]}$.

Our research group has previously demonstrated that the fibronectin splice variants containing the EDA domain is implicated in the pathogenesis of psoriasis. This suggests the interesting question whether the identified splicing genes, LUC7L3, PPIG and SFRS18, contribute to the production of the EDA+ fibronectin variant. We are currently investigating the role of LUC7L3, PPIG and SFRS18 splicing regulatory genes in the production of EDA+ fibronectin, and we aim to identify further differentially spliced mRNA variants contributing to psoriasis pathogenesis.

\section{CONCLUSION}

Taken together, recent comparisons between psoriatic non-involved and involved epidermis dominated largescale gene expression studies related to psoriasis. Relatively few studies have focused on the comparison of gene expression differences between healthy and psoriatic non-involved epidermis samples. Nonetheless, we believe that these experiments are valuable for identifying factors that increase the risk for developing psoriatic plaques. In our microarray studies, we identified several novel candidate genes and molecular patterns that might contribute the formation of typical lesions. The altered expression of EDA+ fibronectin and that of LUC7L3, PPIG and SFRS-18 suggests that some kind of splicing anomalies have an important role in the development of psoriatic symptoms. The exploration of cellular networks related to RNA-maturation processes gave us a deeper insight into the molecular pathogenesis of psoriasis and investigation of the splicing machinery might be a very new approach in this field. Results of wide-scale gene expression studies have provided pioneering advances in psoriasis research as well as in the recognition of different types of non-coding RNAs, including PRINS. This RNA is a long non-coding RNA (lncRNA), and most lncRNAs have been identified in their involvements in the central nervous system and certain tumors ${ }^{[64-69]}$.

The last decade has seen a rapid evolution in largescale gene expression profiling methods. Techniques, such as RNA-Seq and digital gene expression profiling, provide an even greater resolution and wider dynamic range compared to either DD or cDNA microarray. Advancement of methods based on next-generation sequencing has accelerated the accumulation of data, and processing the results requires huge efforts. Thus, validation and interpretation of these newly discovered factors is a very important challenge. Identification of new candidates might establish new therapeutic approaches, which may allow treatment of already existing psoriatic lesions as well as non-involved psoriatic skin by affecting molecular aberrancies, and may lead to the development of prophylactic interventions.

\section{REFERENCES}

1 Nestle FO, Kaplan DH, Barker J. Psoriasis. N Engl J Med 2009; 361: 496-509 [PMID: 19641206 DOI: 10.1056/NEJMra0804595]

2 Roberson ED, Bowcock AM. Psoriasis genetics: breaking the barrier. Trends Genet 2010; 26: 415-423 [PMID: 20692714 
DOI: 10.1016/j.tig.2010.06.006]

3 Gudjonsson JE, Ding J, Li X, Nair RP, Tejasvi T, Qin ZS, Ghosh D, Aphale A, Gumucio DL, Voorhees JJ, Abecasis GR, Elder JT. Global gene expression analysis reveals evidence for decreased lipid biosynthesis and increased innate immunity in uninvolved psoriatic skin. J Invest Dermatol 2009; 129: 2795-2804 [PMID: 19571819 DOI: 10.1038/ jid.2009.173]

4 Hunter HJ, Griffiths CE, Kleyn CE. Does psychosocial stress play a role in the exacerbation of psoriasis? $\mathrm{Br} J$ Dermatol 2013; 169: 965-974 [PMID: 23796214 DOI: 10.1111/bjd.12478]

5 Bowcock AM, Barker JN. Genetics of psoriasis: the potential impact on new therapies. J Am Acad Dermatol 2003; 49: S51-S56 [PMID: 12894126 DOI: 10.1016/S0190-9622(03)01135-6]

6 Bowcock AM. Psoriasis genetics: the way forward. J Invest Dermatol 2004; 122: xv-xvii [PMID: 15175054 DOI: 10.1111/ j.0022-202X.2004.22627.x]

7 Tsoi LC, Spain SL, Knight J, Ellinghaus E, Stuart PE, Capon F, Ding J, Li Y, Tejasvi T, Gudjonsson JE, Kang HM, Allen MH, McManus R, Novelli G, Samuelsson L, Schalkwijk J, Ståhle M, Burden $\mathrm{AD}$, Smith $\mathrm{CH}$, Cork MJ, Estivill X, Bowcock AM, Krueger GG, Weger W, Worthington J, TaziAhnini R, Nestle FO, Hayday A, Hoffmann P, Winkelmann J, Wijmenga C, Langford C, Edkins S, Andrews R, Blackburn H, Strange A, Band G, Pearson RD, Vukcevic D, Spencer CC, Deloukas P, Mrowietz U, Schreiber S, Weidinger S, Koks S, Kingo K, Esko T, Metspalu A, Lim HW, Voorhees JJ, Weichenthal M, Wichmann HE, Chandran V, Rosen CF, Rahman P, Gladman DD, Griffiths CE, Reis A, Kere J, Nair RP, Franke A, Barker JN, Abecasis GR, Elder JT, Trembath RC. Identification of 15 new psoriasis susceptibility loci highlights the role of innate immunity. Nat Genet 2012; 44: 1341-1348 [PMID: 23143594 DOI: 10.1038/ng.2467]

8 Bowcock AM, Shannon W, Du F, Duncan J, Cao K, Aftergut $\mathrm{K}$, Catier J, Fernandez-Vina MA, Menter A. Insights into psoriasis and other inflammatory diseases from large-scale gene expression studies. Hum Mol Genet 2001; 10: 1793-1805 [PMID: 11532989 DOI: 10.1093/hmg/10.17.1793]

9 Képíró L, Széll M, Kovács L, Keszthelyi P, Kemény L, Gyulai R. Genetic risk and protective factors of TNFSF15 gene variants detected using single nucleotide polymorphisms in Hungarians with psoriasis and psoriatic arthritis. Hum Immunol 2014; 75: 159-162 [PMID: 24269700 DOI: 10.1016/ j.humimm.2013.11.006]

10 Bata-Csorgo Z, Hammerberg C, Voorhees JJ, Cooper KD. Kinetics and regulation of human keratinocyte stem cell growth in short-term primary ex vivo culture. Cooperative growth factors from psoriatic lesional $\mathrm{T}$ lymphocytes stimulate proliferation among psoriatic uninvolved, but not normal, stem keratinocytes. J Clin Invest 1995; 95: 317-327 [PMID: 7529261 DOI: 10.1172/JCI117659]

11 Bos JD, De Rie MA. The pathogenesis of psoriasis: immunological facts and speculations. Immunol Today 1999; 20: 40-46 [PMID: 10081229 DOI: 10.1016/S0167-5699(98)01381-4]

12 Szabó K, Bata-Csörgő Z, Dallos A, Bebes A, Francziszti L, Dobozy A, Kemény L, Széll M. Regulatory Networks Contributing to Psoriasis Susceptibility. Acta Derm Venereol 2014 Epub ahead of print [DOI: 10.2340/00015555-1708]

13 Zaba LC, Fuentes-Duculan J, Eungdamrong NJ, Abello MV, Novitskaya I, Pierson KC, Gonzalez J, Krueger JG, Lowes MA. Psoriasis is characterized by accumulation of immunostimulatory and Th1/Th17 cell-polarizing myeloid dendritic cells. J Invest Dermatol 2009; 129: 79-88 [PMID: 18633443 DOI: $10.1038 /$ jid.2008.194]

14 Bata-Csorgo Z, Cooper KD, Ting KM, Voorhees JJ, Hammerberg C. Fibronectin and alpha5 integrin regulate keratinocyte cell cycling. A mechanism for increased fibronectin potentiation of $\mathrm{T}$ cell lymphokine-driven keratinocyte hyperproliferation in psoriasis. J Clin Invest 1998; 101: 1509-1518 [PMID: 9525994 DOI: 10.1172/JCI171]
15 McFadden JP, Basketter DA, Dearman RJ, Kimber IR. Extra domain A-positive fibronectin-positive feedback loops and their association with cutaneous inflammatory disease. Clin Dermatol 2011; 29: 257-265 [PMID: 21496732 DOI: 10.1016/ j.clindermatol.2010.11.003]

16 Széll M, Bata-Csörgo Z, Koreck A, Pivarcsi A, Polyánka H, Szeg C, Gaál M, Dobozy A, Kemény L. Proliferating keratinocytes are putative sources of the psoriasis susceptibilityrelated EDA+ (extra domain A of fibronectin) oncofetal fibronectin. J Invest Dermatol 2004; 123: 537-546 [PMID: 15304094 DOI: 10.1111/j.0022-202X.2004.23224.x]

17 Laskowski MJ. RNA differential display. In: Plant Molecular Biology Manual. In: Gelvin SB, Schilperoort RA, editors. Netherlands: Springer Netherlands, 1997: 33-52 [DOI: 10.100 7/978-94-011-5400-0_3]

18 Liang P, Pardee AB. Differential display. A general protocol. Mol Biotechnol 1998; 10: 261-267 [PMID: 9951706 DOI: 10.1007/BF02740847]

19 Liang P. A decade of differential display. Biotechniques 2002; 33: 338-344, 346 [PMID: 12188186]

20 Velculescu VE, Zhang L, Vogelstein B, Kinzler KW. Serial analysis of gene expression. Science 1995; 270: 484-487 [PMID: 7570003 DOI: $10.1126 /$ science.270.5235.484]

21 Madden SL, Wang CJ, Landes G. Serial analysis of gene expression: from gene discovery to target identification. Drug Discov Today 2000; 5: 415-425 [PMID: 10931659]

22 Villaseñor-Park J, Ortega-Loayza AG. Microarray technique, analysis, and applications in dermatology. J Invest Dermatol 2013; 133: e7 [PMID: 23486432 DOI: 10.1038/ jid.2013.64]

23 Kunz M, Ibrahim SM, Koczan D, Scheid S, Thiesen HJ, Gross G. DNA microarray technology and its applications in dermatology. Exp Dermatol 2004; 13: 593-606 [PMID: 15447719 DOI: 10.1111/j.0906-6705.2004.00243.x]

24 Iyer VR, Eisen MB, Ross DT, Schuler G, Moore T, Lee JC, Trent JM, Staudt LM, Hudson J, Boguski MS, Lashkari $\mathrm{D}$, Shalon D, Botstein D, Brown PO. The transcriptional program in the response of human fibroblasts to serum. Science 1999; 283: 83-87 [PMID: 9872747 DOI: 10.1126/science.283.5398.83]

25 Robbins PB, Sheu SM, Goodnough JB, Khavari PA. Impact of laminin 5 beta 3 gene versus protein replacement on gene expression patterns in junctional epidermolysis bullosa. Hum Gene Ther 2001; 12: 1443-1448 [PMID: 11485635 DOI: 10.1089/104303401750298599]

26 Hinata K, Gervin AM, Jennifer Zhang Y, Khavari PA. Divergent gene regulation and growth effects by NF-kappa $\mathrm{B}$ in epithelial and mesenchymal cells of human skin. Oncogene 2003; 22: 1955-1964 [PMID: 12673201 DOI: 10.1038/ sj.onc.1206198]

27 Blumenberg M. SKINOMICS: Transcriptional Profiling in Dermatology and Skin Biology. Curr Genomics 2012; 13: 363-368 [PMID: 23372422 DOI: 10.2174/13892021280161924 1]

28 Sellheyer K, Belbin TJ. DNA microarrays: from structural genomics to functional genomics. The applications of gene chips in dermatology and dermatopathology. J Am Acad Dermatol 2004; 51: 681-692; quiz 693-696 [PMID: 15523345 DOI: $10.1016 /$ j.jaad.2004.03.038]

29 Blumenberg M. DNA microarrays in dermatology and skin biology. OMICS 2006; 10: 243-260 [PMID: 17069506 DOI: 10.1089/omi.2006.10.243]

30 Baechler EC, Batliwalla FM, Reed AM, Peterson EJ, Gaffney PM, Moser KL, Gregersen PK, Behrens TW. Gene expression profiling in human autoimmunity. Immunol Rev 2006; 210: 120-137 [PMID: 16623768 DOI: 10.1111/ j.0105-2896.2006.00367.x]

31 Oestreicher JL, Walters IB, Kikuchi T, Gilleaudeau P, Surette J, Schwertschlag U, Dorner AJ, Krueger JG, Trepicchio WL. Molecular classification of psoriasis disease-associ- 
ated genes through pharmacogenomic expression profiling. Pharmacogenomics J 2001; 1: 272-287 [PMID: 11911124 DOI: 10.1038/sj.tpj.6500067]

32 Zhou X, Krueger JG, Kao MC, Lee E, Du F, Menter A, Wong WH, Bowcock AM. Novel mechanisms of T-cell and dendritic cell activation revealed by profiling of psoriasis on the 63,100-element oligonucleotide array. Physiol Genomics 2003; 13: 69-78 [PMID: 12644634 DOI: 10.1152/physiolgenomics.00157.2002]

33 Zaba LC, Fuentes-Duculan J, Eungdamrong NJ, JohnsonHuang LM, Nograles KE, White TR, Pierson KC, Lentini T, Suárez-Fariñas M, Lowes MA, Krueger JG. Identification of TNF-related apoptosis-inducing ligand and other molecules that distinguish inflammatory from resident dendritic cells in patients with psoriasis. J Allergy Clin Immunol 2010; 125: 1261-1268.e9 [PMID: 20471070 DOI: 10.1016/ j.jaci.2010.03.018]

34 Nomura I, Gao B, Boguniewicz M, Darst MA, Travers JB, Leung DY. Distinct patterns of gene expression in the skin lesions of atopic dermatitis and psoriasis: a gene microarray analysis. J Allergy Clin Immunol 2003; 112: 1195-1202 [PMID: 14657882 DOI: $10.1016 /$ j.jaci.2003.08.049]

35 Romanowska M, al Yacoub N, Seidel H, Donandt S, Gerken H, Phillip S, Haritonova N, Artuc M, Schweiger S, Sterry W, Foerster J. PPARdelta enhances keratinocyte proliferation in psoriasis and induces heparin-binding EGF-like growth factor. J Invest Dermatol 2008; 128: 110-124 [PMID: 17637826 DOI: $10.1038 /$ sj.jid.5700943]

36 Buerger C, Malisiewicz B, Eiser A, Hardt K, Boehncke WH. Mammalian target of rapamycin and its downstream signalling components are activated in psoriatic skin. Br J Dermatol 2013; 169: 156-159 [PMID: 23398394 DOI: 10.1111/bjd.12271]

37 Goldminz AM, Au SC, Kim N, Gottlieb AB, Lizzul PF. NF-kB: an essential transcription factor in psoriasis. J Dermatol Sci 2013; 69: 89-94 [PMID: 23219896 DOI: 10.1016/ j.jdermsci.2012.11.002]

38 Reischl J, Schwenke S, Beekman JM, Mrowietz U, Stürzebecher S, Heubach JF. Increased expression of Wnt5a in psoriatic plaques. J Invest Dermatol 2007; 127: 163-169 [PMID: 16858420 DOI: $10.1038 /$ sj.jid.5700488]

39 Choi JH, Choi DK, Sohn KC, Kwak SS, Suk J, Lim JS, Shin I, Kim SW, Lee JH, Joe CO. Absence of a human DnaJ protein hTid-1S correlates with aberrant actin cytoskeleton organization in lesional psoriatic skin. J Biol Chem 2012; 287: 25954-25963 [PMID: 22692211 DOI: 10.1074/jbc. M111.313809]

40 Manczinger M, Kemény L. Novel factors in the pathogenesis of psoriasis and potential drug candidates are found with systems biology approach. PLoS One 2013; 8: e80751 [PMID: 24303025 DOI: 10.1371/journal.pone.0080751]

41 Tian S, Krueger JG, Li K, Jabbari A, Brodmerkel C, Lowes MA, Suárez-Fariñas M. Meta-analysis derived (MAD) transcriptome of psoriasis defines the "core" pathogenesis of disease. PLoS One 2012; 7: e44274 [PMID: 22957057 DOI: 10.1371/journal.pone.0044274]

42 Sonkoly E, Bata-Csorgo Z, Pivarcsi A, Polyanka H, Kenderessy-Szabo A, Molnar G, Szentpali K, Bari L, Megyeri K, Mandi Y, Dobozy A, Kemeny L, Szell M. Identification and characterization of a novel, psoriasis susceptibilityrelated noncoding RNA gene, PRINS. J Biol Chem 2005; 280: 24159-24167 [PMID: 15855153 DOI: 10.1074/jbc.M501704200]

43 Mecham RP. Overview of extracellular matrix. Curr Protoc Cell Biol 2001; Chapter 10: Unit 10.1 [PMID: 18228295 DOI: 10.1007/978-3-642-16555-9]

44 Muro AF, Chauhan AK, Gajovic S, Iaconcig A, Porro F, Stanta G, Baralle FE. Regulated splicing of the fibronectin EDA exon is essential for proper skin wound healing and normal lifespan. J Cell Biol 2003; 162: 149-160 [PMID: 12847088 DOI: $10.1083 /$ jcb.200212079]

45 White ES, Baralle FE, Muro AF. New insights into form and function of fibronectin splice variants. J Pathol 2008; 216: 1-14 [PMID: 18680111 DOI: 10.1002/path.2388]

46 Kornblihtt AR, Pesce CG, Alonso CR, Cramer P, Srebrow A, Werbajh S, Muro AF. The fibronectin gene as a model for splicing and transcription studies. FASEB J 1996; 10: 248-257 [PMID: 8641558]

47 White ES, Muro AF. Fibronectin splice variants: understanding their multiple roles in health and disease using engineered mouse models. IUBMB Life 2011; 63: 538-546 [PMID: 21698758 DOI: 10.1002/iub.493]

48 Lohi J, Tani T, Laitinen L, Kangas L, Lehto VP, Virtanen I. Tenascin and fibronectin isoforms in human renal cell carcinomas, renal cell carcinoma cell lines and xenografts in nude mice. Int J Cancer 1995; 63: 442-449 [PMID: 7591246 DOI: 10.1007/BF02331434]

49 Ting KM, Rothaupt D, McCormick TS, Hammerberg C, Chen G, Gilliam AC, Stevens S, Culp L, Cooper KD. Overexpression of the oncofetal Fn variant containing the EDA splice-in segment in the dermal-epidermal junction of psoriatic uninvolved skin. J Invest Dermatol 2000; 114: 706-711 [PMID: 10733677 DOI: 10.1046/j.1523-1747.2000.00871.x]

50 Pellegrini G, De Luca M, Orecchia G, Balzac F, Cremona O, Savoia P, Cancedda R, Marchisio PC. Expression, topography, and function of integrin receptors are severely altered in keratinocytes from involved and uninvolved psoriatic skin. J Clin Invest 1992; 89: 1783-1795 [PMID: 1534817 DOI: 10.1172/JCI115782]

51 Bari L, Bacsa S, Sonkoly E, Bata-Csörgo Z, Kemény L, Dobozy A, Széll M. Comparison of stress-induced PRINS gene expression in normal human keratinocytes and $\mathrm{HaCaT}$ cells. Arch Dermatol Res 2011; 303: 745-752 [PMID: 21750967 DOI: 10.1007/s00403-011-1162-8]

52 Szegedi K, Sonkoly E, Nagy N, Németh IB, Bata-Csörgo Z, Kemény L, Dobozy A, Széll M. The anti-apoptotic protein G1P3 is overexpressed in psoriasis and regulated by the non-coding RNA, PRINS. Exp Dermatol 2010; 19: 269-278 [PMID: 20377629 DOI: 10.1111/j.1600-0625.2010.01066.x]

53 Kurki S, Peltonen K, Laiho M. Nucleophosmin, HDM2 and p53: players in UV damage incited nucleolar stress response. Cell Cycle 2004; 3: 976-979 [PMID: 15254398 DOI: 10.4161/cc.3.8.1015]

54 Szegedi K, Göblös A, Bacsa S, Antal M, Németh IB, BataCsörgő Z, Kemény L, Dobozy A, Széll M. Expression and Functional Studies on the Noncoding RNA, PRINS. Int J Mol Sci 2012; 14: 205-225 [PMID: 23344029 DOI: 10.3390/ ijms14010205]

55 Lin CL, Leu S, Lu MC, Ouyang P. Over-expression of SRcyclophilin, an interaction partner of nuclear pinin, releases SR family splicing factors from nuclear speckles. Biochem Biophys Res Commun 2004; 321: 638-647 [PMID: 15358154 DOI: 10.1016/j.bbrc.2004.07.013]

56 Bourquin JP, Stagljar I, Meier P, Moosmann P, Silke J, Baechi T, Georgiev O, Schaffner W. A serine/arginine-rich nuclear matrix cyclophilin interacts with the C-terminal domain of RNA polymerase II. Nucleic Acids Res 1997; 25: 2055-2061 [PMID: 9153302 DOI: 10.1093/nar/25.11.2055]

57 Hegele A, Kamburov A, Grossmann A, Sourlis C, Wowro S, Weimann M, Will CL, Pena V, Lührmann R, Stelzl U. Dynamic protein-protein interaction wiring of the human spliceosome. Mol Cell 2012; 45: 567-580 [PMID: 22365833 DOI: 10.1016/j.molcel.2011.12.034]

58 Puig O, Bragado-Nilsson E, Koski T, Séraphin B. The U1 snRNP-associated factor Luc7p affects 5' splice site selection in yeast and human. Nucleic Acids Res 2007; 35: 5874-5885 [PMID: 17726058 DOI: 10.1093/nar/gkm505]

59 Sakashita E, Tatsumi S, Werner D, Endo H, Mayeda A. Human RNPS1 and its associated factors: a versatile alternative pre-mRNA splicing regulator in vivo. Mol Cell Biol 2004; 24: 1174-1187 [PMID: 14729963 DOI: 10.1128/MCB.24.3.1174]

60 Zimowska G, Shi J, Munguba G, Jackson MR, Alpatov R, 
Simmons MN, Shi Y, Sugrue SP. Pinin/DRS/memA interacts with SRp75, SRm300 and SRrp130 in corneal epithelial cells. Invest Ophthalmol Vis Sci 2003; 44: 4715-4723 [PMID: 14578391 DOI: $10.1167 /$ iovs.03-0240]

61 Pagani F, Baralle FE. Analysis of Human Splicing Defects Using Hybrid Minigenes. Molecular Diagnostics. Trieste, Italy: International Centre for Genetic Engineering and Biotechnology, Padriciano 99, 2010: 155-169 [DOI: 10.1016/ B978-0-12-374537-8.00011-0]

62 Faustino NA, Cooper TA. Pre-mRNA splicing and human disease. Genes Dev 2003; 17: 419-437 [PMID: 12600935 DOI: 10.1101/gad.1048803]

63 Ward AJ, Cooper TA. The pathobiology of splicing. J Pathol 2010; 220: 152-163 [PMID: 19918805 DOI: 10.1002/path.2649]

64 Martignetti JA, Brosius J. BC200 RNA: a neural RNA polymerase III product encoded by a monomeric Alu element. Proc Natl Acad Sci USA 1993; 90: 11563-11567 [PMID: 8265590 DOI: 10.1073/pnas.90.24.11563]

65 Mus E, Hof PR, Tiedge H. Dendritic BC200 RNA in aging and in Alzheimer's disease. Proc Natl Acad Sci USA 2007; 104: 10679-10684 [PMID: 17553964 DOI: 10.1073/pnas.0701532104]
66 Széll M, Bata-Csörgo Z, Kemény L. The enigmatic world of mRNA-like ncRNAs: their role in human evolution and in human diseases. Semin Cancer Biol 2008; 18: 141-148 [PMID: 18282717 DOI: 10.1016/j.semcancer.2008.01.007]

67 Pollard KS, Salama SR, Lambert N, Lambot MA, Coppens S, Pedersen JS, Katzman S, King B, Onodera C, Siepel A, Kern AD, Dehay C, Igel H, Ares M, Vanderhaeghen P, Haussler D. An RNA gene expressed during cortical development evolved rapidly in humans. Nature 2006; 443: 167-172 [PMID: 16915236 DOI: 10.1038/nature05113]

68 Srikantan V, Zou Z, Petrovics G, Xu L, Augustus M, Davis L, Livezey JR, Connell T, Sesterhenn IA, Yoshino K, Buzard GS, Mostofi FK, McLeod DG, Moul JW, Srivastava S. PCGEM1, a prostate-specific gene, is overexpressed in prostate cancer. Proc Natl Acad Sci USA 2000; 97: 12216-12221 [PMID: 11050243 DOI: 10.1073/pnas.97.22.12216]

69 Bussemakers MJ, van Bokhoven A, Verhaegh GW, Smit FP, Karthaus HF, Schalken JA, Debruyne FM, Ru N, Isaacs WB. DD3: a new prostate-specific gene, highly overexpressed in prostate cancer. Cancer Res 1999; 59: 5975-5979 [PMID: 10606244]

P- Reviewer: Husein-ElAhmed H, Kita K, Lonchin S S- Editor: Song XX L- Editor: A E- Editor: Lu YJ 